\title{
Operability-Based Design of Energy Systems: Application to Natural Gas Utilization Processes
}

\author{
Juan C. Carrasco-Moraga
}

Follow this and additional works at: https://researchrepository.wvu.edu/etd

\section{Recommended Citation}

Carrasco-Moraga, Juan C., "Operability-Based Design of Energy Systems: Application to Natural Gas Utilization Processes" (2017). Graduate Theses, Dissertations, and Problem Reports. 5320.

https://researchrepository.wvu.edu/etd/5320

This Dissertation is protected by copyright and/or related rights. It has been brought to you by the The Research Repository @ WVU with permission from the rights-holder(s). You are free to use this Dissertation in any way that is permitted by the copyright and related rights legislation that applies to your use. For other uses you must obtain permission from the rights-holder(s) directly, unless additional rights are indicated by a Creative Commons license in the record and/ or on the work itself. This Dissertation has been accepted for inclusion in WVU Graduate Theses, Dissertations, and Problem Reports collection by an authorized administrator of The Research Repository @ WVU.

For more information, please contact researchrepository@mail.wvu.edu. 


\title{
Operability-Based Design of Energy Systems: Application to Natural Gas Utilization Processes
}

\author{
Juan C. Carrasco-Moraga
}

\author{
Dissertation submitted to the \\ Benjamin M. Statler College of Engineering and Mineral Resources \\ At West Virginia University \\ In partial fulfillment of the requirements for the degree of \\ Doctor of Philosophy in \\ Chemical Engineering
}

\author{
Dr. Fernando V. Lima, Ph.D., Chair \\ Dr. Richard Turton, Ph.D. \\ Dr. Brian Anderson, Ph.D. \\ Dr. Aaron Noble, Ph.D. \\ Dr. David Vinson, Ph.D. \\ Department of Chemical and Biomedical Engineering \\ Morgantown, West Virginia \\ 2017
}

Keywords: operability, process intensification, bilevel optimization, parallel computing programming, high-dimensional nonlinear systems, natural gas utilization, membrane reactor, natural gas combined cycle, modular systems 


\title{
Abstract \\ Operability-Based Design of Energy Systems: Application to Natural Gas Utilization Processes
}

\author{
Juan C. Carrasco-Moraga
}

Process operability emerged in the last decades as a powerful tool for the design and control of complex chemical processes. The design of such processes is a challenging task as they are represented by nonlinear models with large numbers of differential and algebraic equations that demand high computational effort for their solution. In particular, process operability was proposed as a method for verifying the ability of a process design, defined by the available input set, to reach an achievable output set that considers production targets. However, existing operability methods for nonlinear systems are limited by the problem size that they can address.

In this thesis, a novel operability framework for process design and intensification of high-dimensional nonlinear chemical and energy processes is developed. This proposed framework bridges the gap in the literature by addressing the challenges of process nonlinearity and model size. This framework also broadens the scope of the traditional path of operability approaches for design and control, mainly oriented to obtain the achievable output set from the available input set, and compare the computed achievable output set to a desired output set. In particular, an optimization algorithm based on nonlinear programming tools is formulated for the high-dimensional calculations of the desired input set that is feasible considering process constraints, performance levels, and intensification targets. The high computational effort required for the high- 
dimensional calculations is addressed by the incorporation of bilevel and parallel programming approaches into the classical process operability concepts.

To illustrate the effectiveness of the developed methods, two natural gas utilization processes of different dimensionalities are addressed: i) a catalytic membrane reactor for the direct methane aromatization conversion to benzene and hydrogen, for which an intensified reactor design footprint reduction up to $90 \%$ when compared to the base case is obtained; and ii) a natural gas combined cycle system for power generation, for which a dramatic reduction in size, from 400 to 0.11 [MW], is produced by specifying conditions of the gas and steam turbine cycles, while still keeping the high net plant efficiency between 55 and 56.5 [\%]. These results indicate that this novel operability framework can be a powerful tool for enabling process intensification and modularity. Moreover, results on the implementation of the bilevel and parallel computing methods show a reduction in computational time up to 2 orders of magnitude, when compared to the original results. The results in this thesis have culminated in four peer reviewed publications and four delivered presentations by the time of the defense. 


\section{Dedicated to}

God, my wife Claudia, my sons Pablo and Carlos, my parents Jose Ignacio and Ilda Andrea, and my parents in law Juan Evangelista and Elia Elcira 


\section{Acknowledgments}

I would like to say thank you to many people who participated in making my stay as a Ph.D. graduate student at West Virginia University a memorable and unforgettable experience.

My deepest gratitude to Dr. Fernando V. Lima who gave me the opportunity to join his CODES research group. His friendship, patience, motivation, and continuous support as my research advisor were fundamental pillars of my Ph.D. His knowledge and expertise helped me to make the correct decisions regarding my written publications and presentations. I would also like to thank my Thesis committee members: Prof. Richard Turton, Prof. Brian Anderson, Prof. Aaron Noble, and Dr. David Vinson (VinsonTech LLC), for their insightful comments and guidance, even for the hard questions that encouraged me to explore other perspectives to enrich the results of my research. I would like to extend this gratitude to Prof. Dongxia Liu (UMD) for providing the DMA-MR simulation conditions, Dr. Stephen Zitney (NETL) for attaching technical information on NGCC power plant, and Dr. David Rincon (Postdoctoral fellow, WVU) for his help with the optimization algorithms in MATLAB.

I thank my great office friends Gaurav and Shuyun for their unconstrained help and for the enjoyable times we had during these years. I would like to thank my office, CODES and WVU friends, Xin, Lei, Manish, Taiwo, Prathyusha, Vitor, Rebecca, Nick, Brent, Vitor F., Gilson, Matt, Nirjhar, Joe, Tyler, Jacob, Eilish, Yacine, Zach, Yuan, Anderson, Josh, 
Samson and many others for building a friendly, interesting and comfortable work environment.

My eternal gratefulness to my friends of the Hope Music Band, Trivia Entretenida, Choir, and CMA Church: Amada, Don, Tom, Claudia, Sympa, Lingwei, Valentina, Donald, Phil, Baaba, Jef, Ken, Sergio, Andrew, Cindy, Eric, Lihua, Chuck, Gini, Mary, Marcelita, Qiang, Valerie, Ed, John, Jack, Jim, Marcela, Jimmy, Susan, Scott, Ruth, and Pastors Doug, Corey, and Bruce for their huge emotional and spiritual support in my family's life.

I would like to extend my thankfulness to my awesome Chilean friends, Gloria, Rory, Patricio, Fernando, Alex, Carla, Marcelo, Sergio, Juan, Manuel, Ivan, Ricardo, Eduardo, Rodrigo, Samuel, Alexis, Joel, Daniel, Luis, Pastors: Samuel, Adela, Jose, Alicia, Nelson and Eliana for their incentives to study for a Ph.D., economic support, and constant prayers.

Finally and most importantly, my wife Claudia for supporting me with love all these years, my children Pablo and Carlos who have been a part of this great adventure in the USA, my mother Andrea for advising me to study and make learning a priority in my life, and my Chilean relatives, Juan, Elia, Guillermo, Baldramira, Flor, Andres, Angela, Teresa, and many others for helping me with important details in my family life. 


\section{Table of Contents}

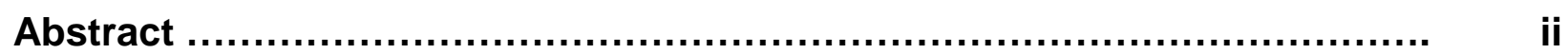

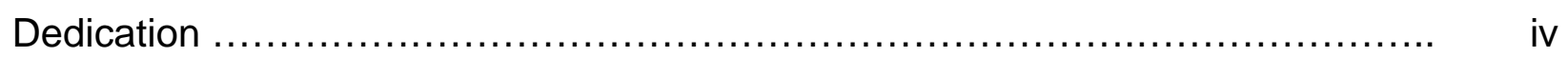

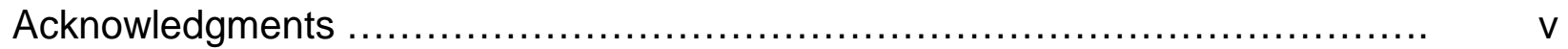

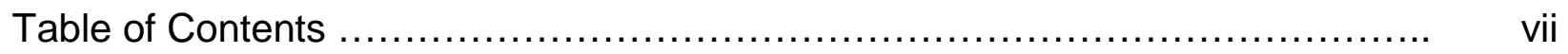

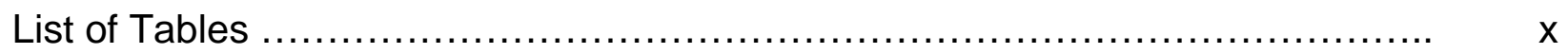

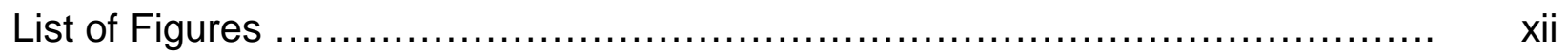

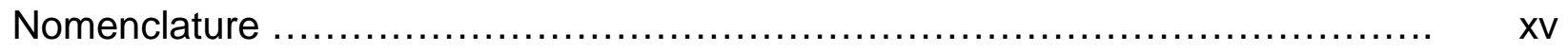

Chapter 1 Introduction ................................................................ $\quad 2$

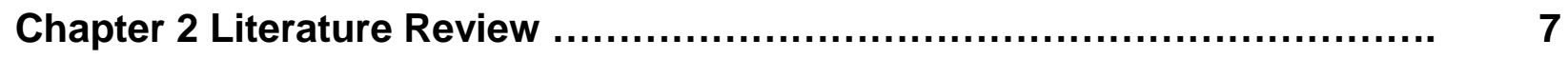

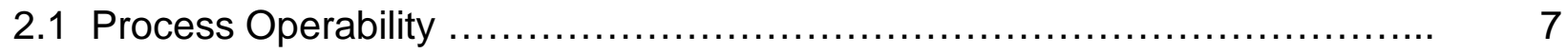

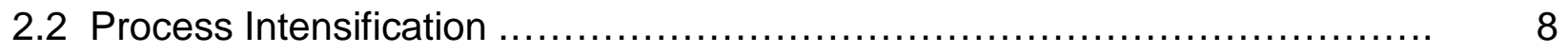

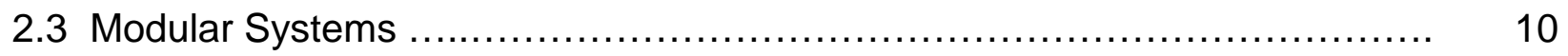

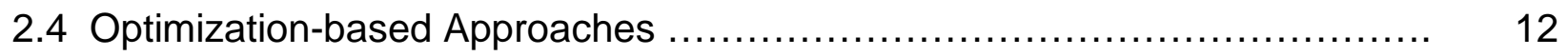

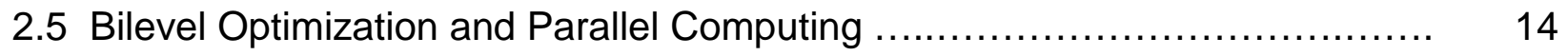

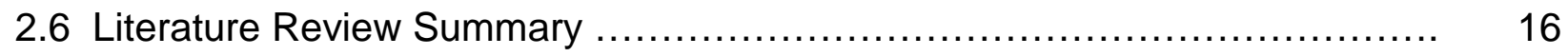

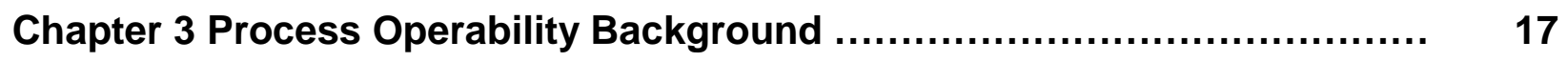

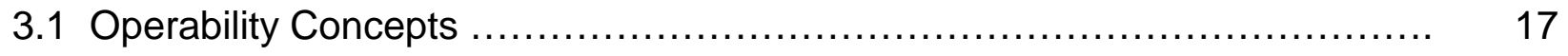

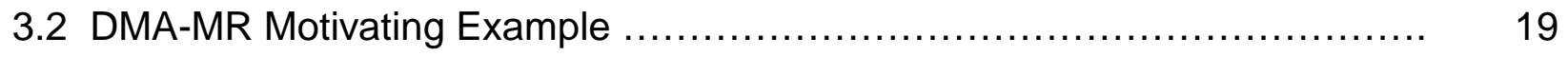

3.2.1 DMA-MR model ............................................... 19

3.2.2 DMA-MR operability analysis .................................... 22

Chapter 4 Novel Operability Approaches for Process Design ................... 29

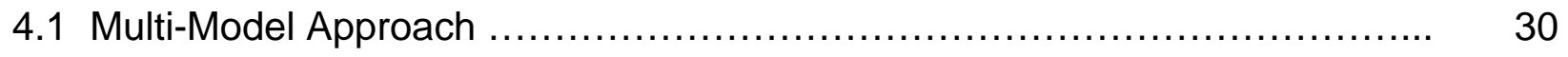


4.1.1 Problem formulation ............................................. 31

4.1.2 DMA-MR case study ........................................... 32

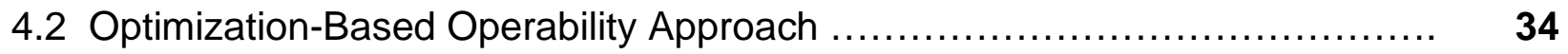

4.2.1 Problem formulation ............................................... 35

4.2.2 DMA-MR case studies ............................................. 37

Chapter 5 Incorporation of Process Intensification into Operability

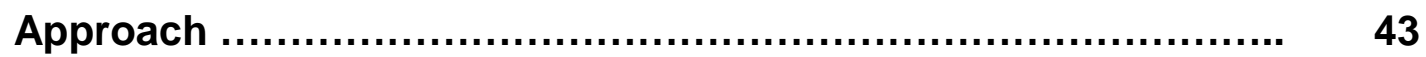

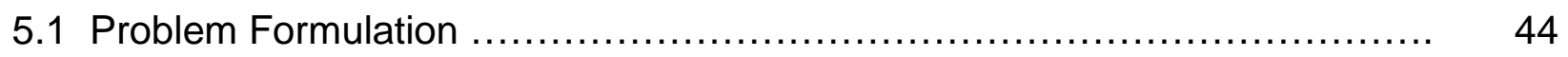

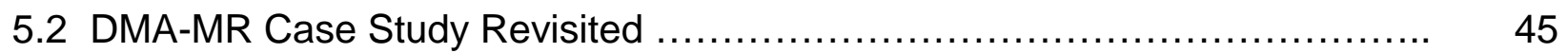

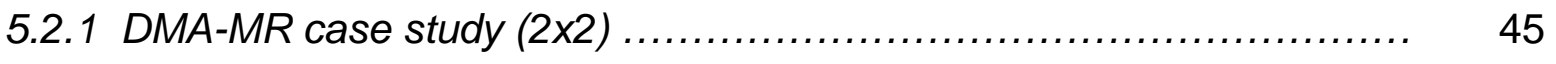

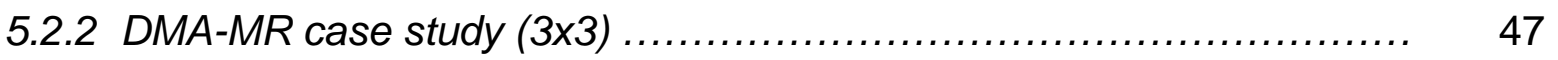

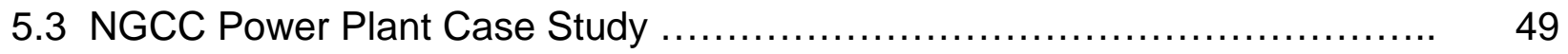

5.3.1 Process description ............................................... 49

5.3 .2 Operability analysis ............................................ 51

5.3.3 Process design and intensification ................................. 53

Chapter 6 Bilevel Operability Framework ......................................... 57

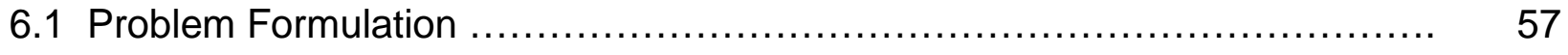

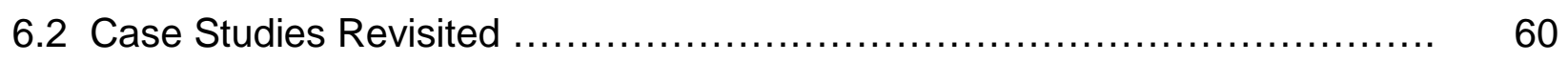

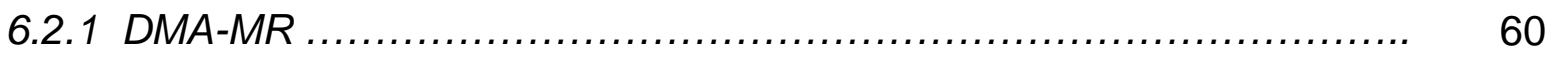

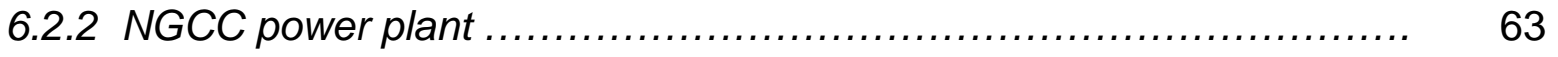

6.3 Exploration of High-Dimensional Application: DMA-MR Case Study (4x4) .... 67

$\begin{array}{ll}\text { Chapter } 7 \text { Operability Approach for Nonlinear High-Dimensional Systems ... } & 77\end{array}$

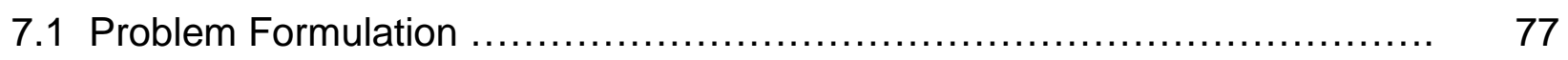

7.1.1 Strategies towards computational time reduction ..................... 77 
7.1.2 Novel operability framework with parallelization .................... 81

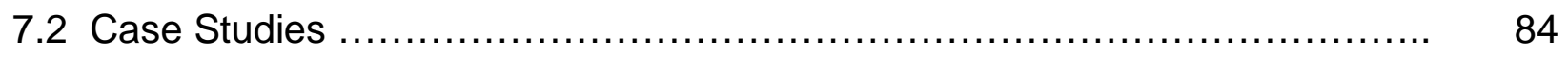

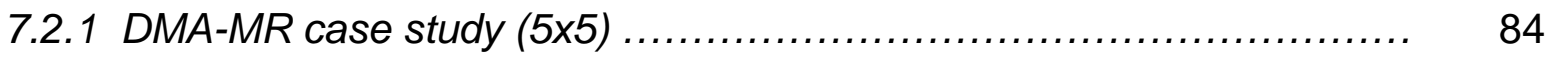

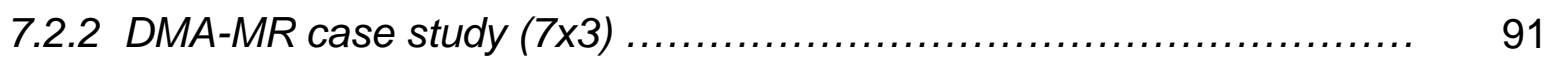

7.2.3 NGCC power plant case study (8x8) ........................... 98

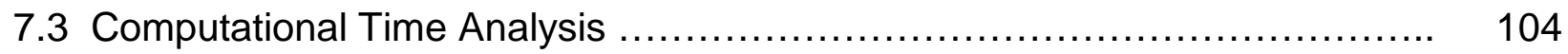

7.3.1 DMA-MR case study ....................................... 104

7.3.2 NGCC power plant case study ................................ 108

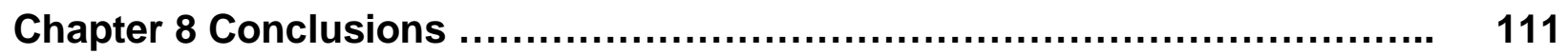

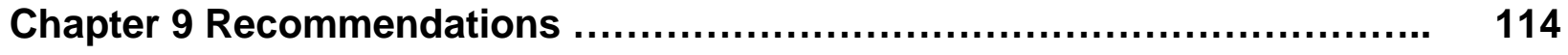

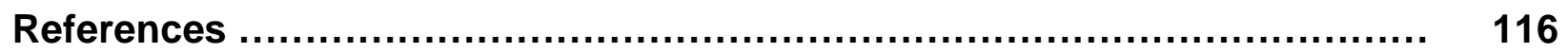




\section{List of Tables}

Table 6.1. Operating conditions for intensified modular NGCC plant ................... 66

Table 6.2. 4-D DMA-MR system: input-output ranges that define the AIS and AOS ..... 67

Table 6.3. 4-D DMA-MR system: achievable ranges for output variables ............... 68

Table 6.4. Computational time for AOS calculations for different DMA-MR subsystems . $\quad 70$

Table 6.5. 4-D DMA-MR system: specified DOS, calculated DIS* and DOS* ranges .... 72

Table 6.6. 4-D DMA-MR system: intensified input and output points for analyzed variables

Table 6.7. Computational time for DIS* calculations for DMA-MR subsystems of different dimensionalities

Table 7.1. Computational time for DIS* calculations for DMA-MR subsystems of different dimensionalities considering modified objective function

Table 7.2. Computational time for DIS* calculations for DMA-MR subsystems of different dimensionalities, for improved objective function and floating point for input initial guess

Table 7.3. 5-D DMA-MR system: input-output ranges that define the AIS and AOS

Table 7.4. 5-D DMA-MR system: specified DOS, calculated DIS* and DOS* ranges ....

Table 7.5. 5-D DMA-MR system: summary of input variables- available and feasible ranges

Table 7.6. 5-D DMA-MR system: summary of output variables- available, desired and feasible ranges

Table 7.7. 5-D DMA-MR system: intensified input and output variables 
Table 7.8. Comparison of computational times for P3 and P4 frameworks for the DIS*

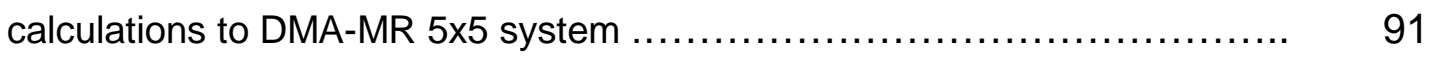

Table 7.9. $7 \times 3$ DMA-MR system: input-output ranges that define the AIS and AOS ..... 92

Table 7.10. 7×3 DMA-MR system: achievable ranges for output variables ............... 92

Table 7.11. 7x3 DMA-MR system: specified DOS, calculated DIS* and DOS* ranges .... 94

Table 7.12. 7x3 DMA-MR system: intensified input and output variables ................ 96

Table 7.13. Comparison of computational time for $\mathrm{P} 3$ and $\mathrm{P} 4$ frameworks associated with the DIS* calculations for DMA-MR 3×3,5×3 and $7 \times 3$ subsystems ....... 97

Table 7.14. NGCC power plant: input-output ranges that define the AIS and AOS ....... 99

Table 7.15. NGCC power plant: comparison between achievable ranges for $2 \times 2$ and $8 \times 8$

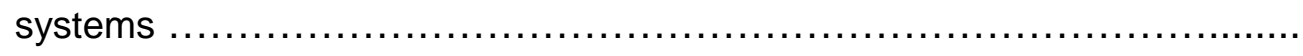

Table 7.16. 8x8 NGCC power plant: achievable and desired output variables ............ 100

Table 7.17. 8x8 NGCC power plant: available and desired input variables ............... 101

Table 7.18. 8x8 NGCC power plant: intensified input-output variables ................... 102

Table 7.19. NGCC power plant: comparison of computational times for P3 and P4 frameworks associated with the DIS* calculations for several subsystems .... 103

Table 7.20. Summary of computational times for DIS* calculations using P4 framework for different DMA-MR subsystems

Table 7.21. Summary of computational times for the DMA-MR $2 \times 2,3 \times 3$ and $4 \times 4$ subsystems using P4 framework for different number of cores .....

Table 7.22. NGCC power plant: comparison of computational times using P4 framework for different subsystems 


\section{List of Figures}

Figure 1.1. Schematic of novel operability framework developed in this thesis ........... 4

Figure 2.1. Parallel computing programming tools in MATLAB ${ }^{\circledR} \ldots \ldots \ldots \ldots \ldots \ldots \ldots \ldots \ldots \ldots \ldots$

Figure 3.1. Schematic of DMA-MR with a cocurrent configuration $\ldots \ldots \ldots \ldots \ldots \ldots \ldots \ldots \ldots . . \ldots \ldots$

Figure 3.2. 2-D DMA-MR system. Input variables: membrane permeance and selectivity (AIS on the left). Process outputs: conversion of methane and production rate of benzene (AOS on the right)

Figure 3.3. 2-D DMA-MR system. Input variables: diameter and length of tube (AIS on the left). Process outputs: conversion of methane and production rate of

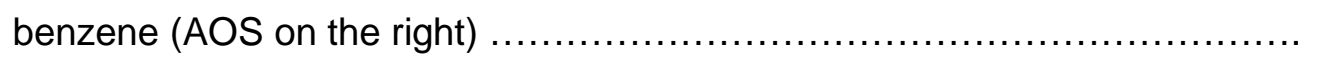

Figure 3.4. 3-D DMA-MR system. Input variables: reactor diameter, length, and membrane selectivity (AIS on the left). Process outputs: conversion of methane, production rates of benzene and hydrogen (AOS on the right) .......

Figure 4.1. Schematic of operability framework for process design

Figure 4.2. 2-D DMA-MR system. Input variables: diameter and length of tube (AIS on the left). Process outputs: conversion of methane and production rate of benzene (AOS and DOS on the right)

Figure 4.3. 2-D DMA-MR system. AOS intersection with DOS (on the left). Calculated

DIS (on the right)

Figure 4.4. 2-D DMA-MR system. DIS* and AIS (on the left). DOS*, DOS and AOS (on the right)

Figure 4.5. 3-D DMA-MR system. AOS and DOS (on the left). DIS* (on the right) .... 40

Figure 5.1. Schematic of operability approach with process intensification 
Figure 5.2. 2-D DMA-MR system. AIS, DIS* and DIS ${ }_{P I}$ (on the left). DOS* and DOS (on the right)

Figure 5.3. Mathematical approach of process operability for intensification

Figure 5.4. 3-D DMA-MR system. Operability sets: DIS* and DIS ${ }_{P I}$ (on the left). DOS* and $\mathrm{DOS}_{P I}$ (on the right)

Figure 5.5. Simplified schematic of NGCC power plant

Figure 5.6. NGCC power plant. Natural gas and HRSG steam flows as inputs (AIS on the left). Net plant power and efficiency as outputs (AOS on the right)

Figure 5.7. NGCC power plant. Operability sets: AIS and DIS* (on the left). AOS, DOS and $\mathrm{DOS}^{*}$ (on the right)

Figure 5.8. NGCC power plant. Operability sets: DIS* and DIS ${ }_{P I}$ (on the left). DOS* and $\operatorname{DOS}_{P I}$ (on the right)

Figure 6.1. Schematic diagram of the bilevel optimization-based operability framework for process intensification

Figure 6.2. 2-D DMA-MR system. Intensified input point and AIS (on the left). Intensified output point, AOS and DOS (on the right)

Figure 6.3. 3-D DMA-MR system. Operability sets: AIS, DIS* and $\mathrm{u}_{P I}^{*}$ (on the left). AOS, DOS* and $_{\mathrm{y}_{P I}^{*}}$ (on the right)

Figure 6.4. NGCC power plant. Intensified input point and AIS (on the left). Intensified output point, AOS and DOS (on the right)

Figure 6.5. Towards modular NGCC plant: intensified input point and AIS (on the left). Intensified output point, AOS and DOS (on the right)

Figure 6.6. 4-D DMA-MR system. AOS and DOS

Figure 6.7. 4-D DMA-MR system. DIS* 
Figure 6.8. 4-D DMA-MR system. DOS and DOS* (zoomed version)

Figure 7.1. Computational time using desktop system with local workers for parallelization 80

Figure 7.2. Schematic diagram of the bilevel optimization and parallel programmingbased operability framework for process intensification

Figure 7.3. 7x3 DMA-MR system. AOS and DOS

Figure 7.4. 7x3 DMA-MR system. DOS and DOS* (zoomed version)

Figure 7.5. Trendlines for the relationship between DMA-MR subsystem dimensionality and computational time for a cluster with 63 cores

Figure 7.6. Power law trendlines for the relationship between number of cores and computational time for the DMA-MR $2 \times 2,3 \times 3$ and $4 \times 4$ systems

Figure 7.7. Exponential trendline for the relationship between NGCC subsystem dimensionality and computational time for a cluster with 63 cores 


\section{Nomenclature}

Notation

A

C

$\mathbf{C}_{1}$

d

D

f

F

g

$\mathbf{h}_{1}$

$\mathbf{h}_{2}$

I

$k_{1}$

$k_{1}^{\prime}$

$k_{2}$

$k_{2}^{\prime}$

$L$

M

$\mathbf{M}^{-1}$

$\mathrm{N}$

NPP

$P$ cross-sectional area of the reactor $\left[\mathrm{cm}^{2}\right]$

concentration $\left[\mathrm{mol} / \mathrm{cm}^{3}\right]$

nonlinear constraint map for target specifications

vector of the disturbances

diameter [cm]

nonlinear map for the time derivative of the state variables

molar flow rate $[\mathrm{mol} / \mathrm{s}]$

nonlinear map for vector of output variables

vector of equality constraints

vector of inequality constraints

number of input variables for computational time calculations

reaction rate constant for step $1\left[\mathrm{~s}^{-1}\right]$

inverse reaction rate constant for step 1 [cm³/s.mol]

reaction rate constant for step $2\left[\mathrm{~s}^{-1}\right]$

inverse reaction rate constant for step 2 [ $\mathrm{cm}^{3} / \mathrm{s}$. mol]

length of reactor $[\mathrm{cm}]$

process model matrix

process model inverse matrix

number of workers or cores in a cluster

net plant power [MW]

pressure [atm] 


\begin{tabular}{|c|c|}
\hline Pltargets & process intensification targets [in appropriate units] \\
\hline$Q$ & permeance $\left[\mathrm{mol} /\left(\mathrm{s} . \mathrm{m}^{2} . \mathrm{atm}^{1 / 4}\right]\right.$ \\
\hline$r_{1}$ & reaction rate for step $1\left[\mathrm{~mol} / \mathrm{s} . \mathrm{cm}^{3}\right]$ \\
\hline$r_{2}$ & reaction rate for step $2\left[\mathrm{~mol} / \mathrm{s} . \mathrm{cm}^{3}\right]$ \\
\hline $\mathbb{R}_{\geq 0}$ & set of non-negative numbers \\
\hline $\mathrm{S}$ & dimension of a square system \\
\hline $\mathrm{T}$ & computational time [sec] \\
\hline $\mathbf{u}$ & vector of input variables \\
\hline $\mathrm{u}$ & element of the vector of input variables \\
\hline$\dot{\mathbf{u}}$ & time derivative of the vector of input variables \\
\hline $\mathbb{U}$ & domain of input variables \\
\hline $\mathbb{U}_{k}$ & domain of input points in the DIS* \\
\hline $\mathbf{x}$ & state variables of the process \\
\hline$\dot{\mathbf{x}}$ & time derivative of vector of state variables \\
\hline $\mathbf{y}$ & vector of output variables \\
\hline $\mathrm{y}$ & element of the vector of output variables \\
\hline$z$ & differential length $[\mathrm{cm}]$ \\
\hline
\end{tabular}

\section{Acronyms}

AOS achievable output set

AIS available input set

DIS desired input set

DIS $^{*} \quad$ feasible desired input set

DMA direct methane aromatization 


$\begin{array}{ll}\text { DOS } & \text { desired output set } \\ \text { DOS }^{*} & \text { feasible desired output set } \\ \text { EDS } & \text { expected disturbance set } \\ \text { LP } & \text { linear programming } \\ \text { MR } & \text { membrane reactor } \\ \text { NGCC } & \text { natural gas combined cycle } \\ \text { NLP } & \text { nonlinear programming }\end{array}$

\section{Greek Letters}

$\begin{array}{ll}\propto_{\mathrm{H}_{2} / c} & \text { selectivity between } \mathrm{H}_{2} \text { and component } c \text { [dimensionless] } \\ \varepsilon & \text { relative error between eigenvalues for different models [\%] } \\ \lambda_{i} & \text { eigenvalue for operating region } i \text { [dimensionless] } \\ \Omega & \text { objective function for process intensification [in appropriate units] } \\ \varphi_{w} & \text { objective function for parallel programming [in appropriate units] } \\ \emptyset_{\mathrm{k}} & \text { objective function to calculate the DIS from the DOS [dimensionless] } \\ \psi & \text { objective function for bilevel optimization [in appropriate units] }\end{array}$

\section{Subscripts}

c component in the feed and/or permeate

$\mathrm{CH}_{4} \quad$ methane

$\mathrm{C}_{2} \mathrm{H}_{4} \quad$ ethylene

$\mathrm{C}_{6} \mathrm{H}_{6} \quad$ benzene

$\mathrm{H}_{2} \quad$ hydrogen

i input variable index 


$\begin{array}{ll}j & \text { output variable index } \\ k & \text { point index for the points in the grid } \\ l & \text { point index for process intensification space } \\ m & \text { number of input variables } \\ \text { MR } & \text { membrane reactor } \\ n & \text { number of output variables } \\ \text { NGCC } & \text { natural gas combined cycle } \\ o & \text { initial point for optimization } \\ p & \text { total number of points in the grid } \\ P I & \text { element, set, or vector for process intensification } \\ q & \text { total number of points that satisfy the level of performance inside the DOS } \\ s & \text { shell side } \\ t & \text { tube side } \\ w & \text { point index for the workers or cores } \\ w s & \text { number of workers or cores }\end{array}$

\section{Superscripts}

optimal point, set, or vector

max maximum

min minimum 


\section{Operability-Based Design of Energy Systems:}

\section{Application to Natural Gas Utilization Processes}




\section{Chapter 1 Introduction}

In the recent years, due to the advent of the shale gas revolution, the U.S. has incremented the annual production of processed natural gas by approximately $21 \%$ from 16.3 to 19.8 trillion cubic feet (EIA, 2016a). In fact, the U.S. Energy Information Administration (EIA, 2016b) reported that the year of 2016 was the first year in which electricity generation by natural gas power plants exceeded the amount by their coalbased counterparts, with $33 \%$ and $32 \%$ of the share, respectively. These abundant amounts of shale/natural gas would require techno-economic viability analysis to assess novel and improved natural gas utilization processes that produce heat, power, fuels and chemicals. There are over 2,000 companies associated with the U.S. natural gas industry, in which over 530 plants are dedicated to natural gas processing. For such systems, the U.S. Gas Processors Association mentioned that one of the key areas of research consists of the development of specialized software tools that can provide technical and economic assessment in the natural gas processing and utilization facilities (GPA, 2010). However, the design and control of such processes is a challenging task as these systems are typically operated in a highly constrained and integrated environment that is represented by complex large-scale and nonlinear models.

In the systems community, process operability emerged in the last decades as a powerful tool for the design of chemical processes, including a variety of academic and industrial-scale applications (Georgakis et al., 2003; Lima et al., 2010a). Process operability was proposed as a method for the design and control of chemical processes 
that can verify the ability of an available input set (AIS) to reach an achievable output set (AOS) that considers production targets, through a linear/nonlinear mapping determined by first-principles models (Vinson and Georgakis, 2000). In this thesis, a novel operabilitybased approach is introduced to calculate the desired sets for operation of input variables considering process design and intensification goals. The approach broadens the scope of the traditional path of operability methods for design and control, mainly focused on obtaining the AOS from the AIS. This novel operability framework allows the computation of the desired input set (DIS) from the desired output set (DOS), considering process constraints and intensification targets that are embedded in a formulated nonlinear programming problem.

The objective in this research is the derivation of improved operability-based approaches to address systems that are described by high-dimensional and nonlinear models. The proposed operability methods will focus on large-scale energy systems in general, and natural gas utilization processes in particular. The specific aims for this thesis are:

> Specific aim \#1: Establish a multi-model operability approach for high-dimensional nonlinear systems. The approach involves linear operability calculations using linearized versions of the process model at different operating regions.

> Specific aim \#2: Develop a fully nonlinear method for high-dimensional systems. The approach entails plantwide operability analysis employing nonlinear optimization tools.

> Specific aim \#3: Apply the developed approaches to natural gas utilization processes. The approach consists of the implementation of the developed methods to address first-principles, systems-level models for the processes of interest, including: (i) a catalytic membrane reactor (MR) for the direct methane aromatization (DMA) conversion to benzene and hydrogen; and (ii) a natural gas combined cycle (NGCC) system for power generation. 
The main results of this thesis correspond to: (i) formulation of a bilevel optimization-based operability approach that considers process design constraints, performance levels and intensification targets in one step; (ii) extension of the application domain of classical operability approaches by using process simulator runs for obtaining input-output relationships in addition to first-principles models; and (iii) development of an operability framework to address high-dimensional and nonlinear systems by employing parallel programming tools to efficiently tackle computational time challenges. Figure 1.1 presents a schematic of the novel operability framework developed in this thesis. In this framework, using a laboratory-scale DMA-MR and a simulated large-scale NGCC system, design conditions are obtained for process intensification towards modularity.

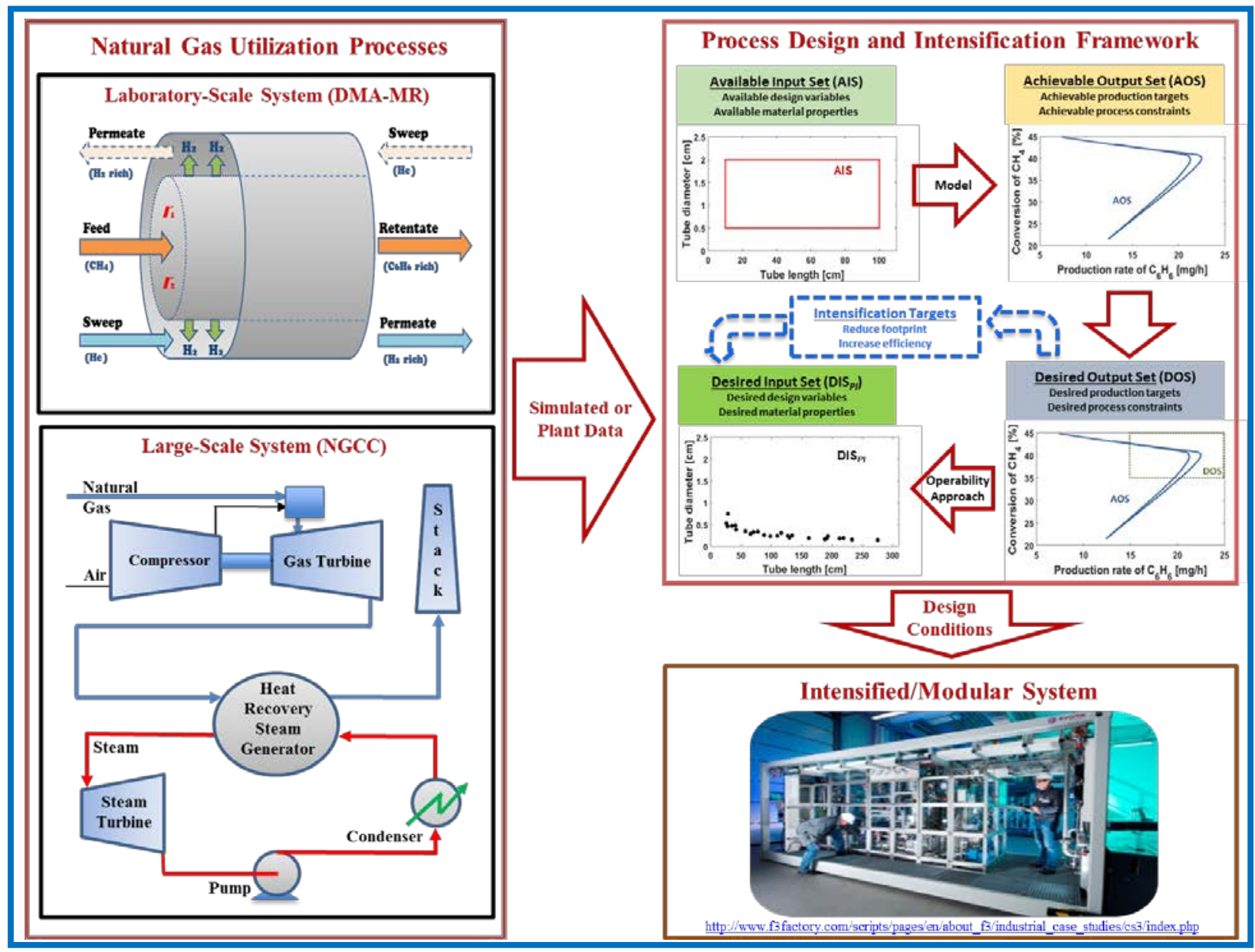

Figure 1.1. Schematic of novel operability framework developed in this thesis 
The contributions of this thesis have resulted in the following products:

Peer reviewed papers:

> Carrasco J. C. and Lima F. V. "New directions on process operability: bilevel and parallel programming approaches for process intensification and modularity of nonlinear and high-dimensional energy systems". In preparation for publication.

D Carrasco J. C. and Lima F. V. "Novel operability-based approach for process design and intensification: application to a membrane reactor for DMA". AIChE J. 2017; 63(3): 975-983.

$>$ Carrasco J. C. and Lima F. V. "Operability-based approach for process design, intensification, and control: application to high-dimensional and nonlinear membrane reactors". In proceedings of the FOCAPO/CPC 2017.

> Carrasco J. C. and Lima F. V. "An optimization-based operability framework for process design and intensification of modular gas utilization systems". Accepted for publication in Comput. Chem. Eng. 2016; DOI: CACE-5645.

> Carrasco J. C. and Lima F.V. "Nonlinear operability of membrane reactors for direct methane aromatization (DMA)", In Proceedings of the IFAC ADCHEM 2015.

Oral presentations:

> Carrasco J. C., Vinson D. R., and Lima F.V. "New directions on process operability: bilevel and parallel programming approaches for process intensification and modularity". Accepted for presentation at AIChE Annual Meeting, Minneapolis, Minnesota, October 2017.

> Carrasco J. C. and Lima F.V. "Novel optimization-based approach for process design and intensification of high-dimensional modular systems". Presented at AlChE Annual Meeting, San Francisco, California, November 2016.

> Carrasco J. C. "An optimization-based operability framework for process design and intensification of complex chemical processes". Presented at Procter \& Gamble's $26^{\text {th }}$ Annual FIRST Conference, Cincinnati, Ohio, September 2016.

> Carrasco J. C. and Lima F.V. "Multi-model operability approach as a tool for process intensification". Presented at AIChE Annual Meeting, Salt Lake City, Utah, November 2015.

> Carrasco J. C., Liu D., and Lima F.V. "Modeling and nonlinear operability analysis of a membrane reactor for direct methane aromatization". Presented at AlChE Annual Meeting, Georgia, Atlanta, November 2014. 
The outline for the remaining chapters of this thesis includes a literature review, which is presented first. Then, the process operability background is described, followed by a motivating example associated with the DMA-MR case study. Next, a novel operability approach for the calculation of the DIS is proposed, including multi-model and optimization-based operability methods for process design. The incorporation of process intensification into operability is then performed, and the extended method is implemented for the NGCC power plant application using process simulator runs in Aspen. As a new framework, an integrated operability approach employing bilevel optimization is presented in the next chapter, and the previous case studies are revisited. Finally, an operability method employing parallel programming for intensification of nonlinear systems is presented as a tool to reduce computational time issues, followed by the conclusions and recommendations for future work. 


\section{Chapter 2 Literature Review}

This chapter presents an overview of the reviewed literature to perform this research, including the main topics of process operability, process intensification, modular systems, optimization-based approaches, bilevel optimization, and parallel computing.

\subsection{Process Operability}

Process operability emerged in the early 2000 s as a powerful tool for the design and control of chemical processes, including a variety of academic and industrial-scale applications from companies such as Air Products and Chemicals and DuPont (Lima et al., 2010a; Georgakis et al., 2003). In particular, operability enables the systematic verification of a design's ability to achieve the feasible region of operation for control in the presence of disturbances and provides a comparative measure to rank competing system designs (Vinson and Georgakis, 2000). Several systems studies that addressed the development and implementation of operability approaches are available in the literature. Specifically for high-dimensional systems, operability calculations using a response surface model-based approach have been performed (Georgakis and Li, 2010). In this work, the authors pointed out the computational limitations of the method for highdimensional applications due to the size of the polynomial model typically used. To address this challenge, the authors proposed the selection of a finite number of points that provide an adequately accurate representation of the system. Also, several efforts have been performed in the linear and high-dimensional operability field. Specifically, 
based on iterative and optimization algorithms, operability methods were applied to complex chemical processes that are described by linear models (Lima and Georgakis, 2008; Lima and Georgakis, 2010). Process flexibility has been recognized as an important component of operability. Simply stated, it focuses on finding the feasible region of operation in the presence of disturbances (Lima et al., 2010b). Using operability and flexibility-based methods, the computation of the process design space was performed (Boukouvala et al., 2010). This space is comprised of the parameter boundaries within which the feasibility of a process is guaranteed. This method employed data-based models (i.e., black box) for the representation of high-dimensional processes. In particular, response surface methodology and Kriging were implemented to allow the representation of the effects that the input parameters have on the outputs of the process. Also related to a Kriging-based approach for flexibility analysis, the mapping of the feasible region of operation for black-box processes with minimum sampling was computed for a roller compactor application (Boukouvala and lerapetritou, 2012). The same group presented the implementation of computational methods for a predictive study of powder mixing for the pharmaceutical and other solids handling industries (Boukouvala et al., 2012). In this application, population balance modeling and discreteelement methods were explored due to their high computational efficiency for the use for instance in process design. In process design, a connection between process integration and process intensification at the design stage using a reaction-separation-recycle processes was recently studied by Baldea (2015). 


\subsection{Process Intensification}

There are several definitions of process intensification considered in the literature. This thesis follows the definition of process intensification by Ramshaw and Stankiewicz (Ramshaw, 1995; Stankiewicz and Moulin, 2000) as a strategy for making dramatic reductions in the size of a chemical process so as to reach a given production objective. In the last decades, MRs have emerged as examples of intensified processes as they combine reaction and separation unit operations in one process improving the system efficiency, lowering the cost and minimizing the environmental footprint (Drioli et al., 2011; Brunetti et al., 2012). MRs allow higher conversions than conventional packed-bed reactors due to the reaction equilibrium shift towards the products caused by the selective removal of one of these products through the membrane. In the DMA-MR case, the focus is on the equilibrium-limited production of hydrogen $\left(\mathrm{H}_{2}\right)$ and benzene $\left(\mathrm{C}_{6} \mathrm{H}_{6}\right)$ from methane $\left(\mathrm{CH}_{4}\right)$ using a catalytic membrane reactor. Specifically in the DMA process, the reaction equilibrium is shifted towards products as $\mathrm{H}_{2}$ is removed through a $\mathrm{H}_{2}$-selective membrane (ion transport-based). Restrictions on process target specifications, such as reaction conversions and production rates, as well as multiple challenges associated with controlling temperatures and compositions, make the problem of designing and controlling MRs for the DMA process a challenging area of research. Several examples using membrane processes for intensification purposes were presented in the literature. For example, the conversion of lignocellulose to biofuels in a biorefinery and the use of membranes for water treatment plants were addressed (Sirkar et al., 2015). Also for waste water treatment, different reactors and designs for photocatalytic processes were 
compared, with the MR scoring the highest performance in terms of treatment efficiency (Leblebici et al., 2015). However, MR systems studies lack of systematic approaches to determine the output (e.g., specifications on reaction conversions and production rates) ranges that are achievable, considering the limited ranges available for the process inputs (e.g., operational variables and membrane characteristics) due to the physical limitations of the process design. Although the reported studies above did not consider footprint reduction for MRs, process intensification could offer dramatic improvements in terms of ratio of production capacity to equipment size towards enabling modular process designs.

\subsection{Modular Systems}

Modular processes offer an alternative in which several technologies could be tested and implemented in a fast and decentralized manner, including shorter product life cycles (Wörsdörfer et al., 2015). A modular plant was recently defined as the process equipment, instrumentation, valves, piping components, and electrical wiring that are mounted within a structural framework (Roy, 2017). A study including a fifteen year record of global natural gas flaring estimated that in the year of 2008 , flared gas represented $21 \%$ of the natural gas consumption in the U.S. This flared gas, typically in isolated regions, could be better utilized providing a potential positive economic impact of approx. US\$ 68 billion (Elvidge et al., 2009). In particular, modular technologies could provide an alternative for the utilization of the stranded shale/natural gas deposits at the site. Several efforts have been conducted in the literature to analyze modular systems. For example, offshore operations have been designed and optimized for the integration of a natural 
gas-fired power plant into a floating structure (Kvamsdal et al., 2010). NGCC process intensification studies were presented associated with a post-combustion $\mathrm{CO}_{2}$ capture process by chemical absorption (Joel et al., 2014; Joel et al., 2015; Wang et al., 2015). All of these reported studies considered NGCC plant sizes larger than 450 [MW]. Also, modeling and efficiency improvement studies were performed for NGCC systems with carbon capture (Nord et al., 2009; Adams and Barton, 2010; Bernier et al., 2010; Liese and Zitney, 2013; Manassaldi et al., 2014). In general, the main focus of these studies was on modeling, simulation and optimization of plants with net power generation greater than 400 [MW]. A comprehensive economic overview of small modular reactors for nuclear power was also presented (Locatelli et al., 2014), including the economic challenges of downsizing these reactor units. Also, a recent review from process industries (Lier et al., 2016) highlighted the benefits of implementing modular processes regarding flexibility, shorter lifecycle and mobility aspects. Furthermore, workshops by the U.S. government agencies (NSF, 2014; DOE, 2015) have emphasized the need for systematic approaches for the incorporation of process and heat integration into the early stages of technology development of new modular systems that combine unit operations and intensified steps. An example of an NGCC modular unit for the production of power at the site, employing transportable technologies, was reported with a net plant power of approximately 50 [MW] (Kehlhofer et al., 2009) and net plant efficiency higher than $50 \%$. Modular natural gas power plants using only a gas turbine, called micro gas turbines, can generate electricity in ranges between $30-1000$ [kW], with electrical efficiency on a lower heating value (LHV) basis between $26-33$ [\%], and temperature of exhaust gases of approx. 550 [K] (Capstone, 2016; NaturalGas, 2016). For micro steam turbines dedicated 
to electricity generation, the minimum generator output is around $80[\mathrm{~kW}]$ and the requested input temperature range of steam is between $700-850$ [K] (G-Team A.S., 2016). Also, a typical steam power plant converts $35-40$ [\%] of the fuel energy to electricity (Kreith and Goswami, 2007). To enable smaller modular NGCC designs, a critical issue yet to be addressed would be the integration between gas and steam turbines for the improvement of thermodynamic and net plant efficiencies.

\subsection{Optimization-based Approaches}

Regarding publications associated with optimization of natural gas utilization processes, specific systems have been studied by Floudas and coworkers (Baliban et al., 2010a; Baliban et al., 2010b; Baliban et al., 2012; Baliban et al., 2013). These studies were focused on hybrid natural gas processes with biomass and coal for the production of liquid fuels. The authors employed a mixed-integer nonlinear optimization framework to address high-dimensional systems. Optimization-based approaches to enable operability and flexibility studies of nonlinear systems have also been addressed in the literature. Particularly, the effects of pressure swing adsorption decision variables, such as the duration of the processing steps, on the operability of the system have been investigated (Khajuria and Pistikopoulos, 2013). In this study, the objective function was to maximize the expected hydrogen recovery. Other developed algorithms associated with flexibility and optimization under uncertainty can be found in references (Dominguez and Pistikopoulos, 2013; Liu et al., 2009). Finally, regarding flexibility analysis for the integration of process design and control under uncertainty, a method entitled embedded 
control optimization was proposed (Malcolm et al., 2007; Moon et al., 2011a; Moon et al., 2011b). These last contributions focused on the process dynamics, leaving aside the use of the developed approach for process design assessment. A framework was introduced for general nonlinear processes applying the concept of incremental dissipativity (Santoso et al., 2012). This framework can be used to analyze operability of the process around different equilibrium points and reference trajectories within a given operating window. Additionally, a network-based approach for plantwide operability assessment was introduced (Setiawan and Bao, 2012). This approach focused on a microscopic level network analysis, in which each nonlinear process unit was modeled using individual mass and energy balances. The authors also indicated the challenge of obtaining networks for certain types of nonlinear systems. Approaches have also been proposed to quantify the attainable region for process networks (Conner and Manousiouthakis, 2013). In particular, a case study for the vapor-liquid equilibrium-based separation of a ternary azeotropic mixture was used to illustrate the method for determination of the attainable regions. In this work, the authors derived process models based on input-output relationships and parameter estimation from experimental data using nonlinear optimization. For conversion of hybrid coal, biomass, and natural gas to liquid fuels, a mixed-integer nonlinear optimization problem was formulated for the improvement of a thermochemical-based conversion process (Baliban et al., 2012). Also related to optimization studies for the conversion of natural gas to liquid fuels, a process synthesis framework was developed (Baliban et al., 2013). This framework considered a mixedinteger nonlinear optimization model to address high-dimensional systems. However, none of these optimization studies accounted for the operability aspects of the process. 
An overview on process modeling techniques and the areas of application of dynamic simulation associated with the natural gas processing industry can be found in reference (Mokhatab and Poe, 2012).

\subsection{Bilevel Optimization and Parallel Computing}

In the mathematical optimization field, bilevel programming formulations were proposed as an alternative for a special class of optimization problems that present two levels with independent objective functions (Bard, 1998; Colson et al., 2007; Sinha et al., 2014). Bilevel programming tools can be employed to solve complex nested problems that are computationally intensive in a streamlined manner (Mathieu et al., 1994; Yin, 2000; Sinha et al., 2013). The main application fields of bilevel optimization thus far were game theory and mathematical programming, thus providing an opportunity for the formulation of this type of optimization problems for process design and intensification of complex chemical and energy systems. Parallel computing is a tool that can allow the solution of optimization problems associated with chemical systems that have never been tackled due to computational time limitations (High and LaRoche, 1995). During the last decades, parallel computing has been one of the pillars of the development of supercomputers. Parallel computing can handle millions of simultaneous calculations allowing huge computational time reductions proportional to the employed number of cores or workers. In publications reported by Biegler and coworkers (Chen et al., 2013; Weng et al., 2015; Ma et al., 2016), it is demonstrated that multi-core processor implementations show significant computational reductions for large-scale equation- 
oriented models with a large number of nonlinear equations. Also, for dynamic simulation of molecular weight distribution of multisite polymerizations, the conventional serial computation can be decomposed into several parallel threads, in which the multi-core parallelization can greatly reduce the computational time. A mixed-integer programming (MIP) model using parallel computing was also employed in the analysis of the scheduling of chemical manufacturing facilities (Velez and Maravelias, 2013). In this study, the authors concluded that although the use of parallel computing programming alone is not sufficient to address highly complicated MIP problems, the design of adequate algorithms that harness computational resources can enable a significant enhancement in terms of computational time. Also, a parallel approach to improve an optimal control problem was employed (Fesko, 2012). Here the author concluded that a significant gain in speed was achieved when the optimization problem was parallelized to find the optimal catalyst blend along the length of the reactor to produce benzene from methylcyclopentane. Parallel computing programming in MATLAB allows the simultaneous use of multiple computer resources to help reducing computational time issues (MathWorks $®, 2017$ ). The following diagram (see Figure 2.1) shows the available parallel computing toolbox capabilities in MATLAB. 


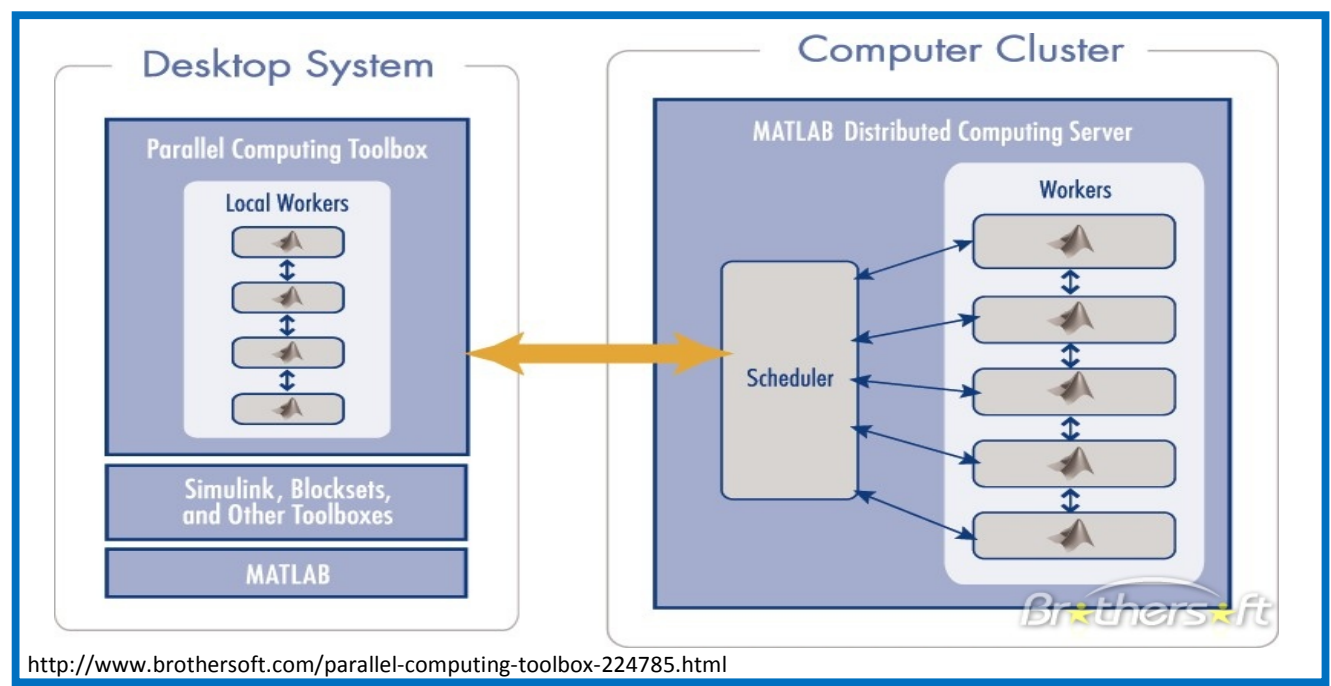

Figure 2.1. Parallel computing programming tools in MATLAB ${ }^{\circledR}$

\subsection{Literature Review Summary}

On the basis of the literature review, gaps have been identified that provide the following research opportunities: (i) extension of process operability approaches to enable process intensification of natural gas conversion systems; (ii) exploration of DMA-MR and NGCC systems for the production of benzene, and power generation, respectively, for process intensification towards the construction of modular designs; (iii) investigation of a bilevel optimization method that can help integrate the optimization-based operability calculations in one step considering defined process design constraints, target specifications, performance levels, and intensification targets; and (iv) employment of parallel computing programming as a novel tool for operability as the key to tackle the challenge of computational time limitations for the design and intensification of nonlinear high-dimensional systems. The following chapters outline the developed operability approaches to fill these identified gaps. 


\section{Chapter 3 Process Operability Background}

This chapter presents the process operability concepts focusing on the interaction between input and output variables. Also, two motivating examples associated with the DMA-MR system are studied.

\subsection{Operability Concepts}

The main contributions on process operability have been detailed in Georgakis et al. (2003), Lima and Georgakis (2008), Lima and Georgakis (2010), Georgakis and Li (2010), Lima et al. (2010a,b). Process operability enables the verification of a design's ability to achieve the feasible region of operation associated with process specifications, considering process models of different complexities. The process model (M) that describes the relationship between the input and output variables can be represented by (Vinson and Georgakis, 2000):

$$
\mathbf{M}=\left\{\begin{array}{l}
\dot{\mathbf{x}}=\mathbf{f}(\mathbf{x}, \mathbf{u}, \mathbf{d}) \\
\mathbf{y}=\mathbf{g}(\mathbf{x}, \mathbf{u}, \mathbf{d}) \\
\mathbf{h}_{\mathbf{1}}(\dot{\mathbf{x}}, \mathbf{x}, \mathbf{y}, \dot{\mathbf{u}}, \mathbf{u}, \mathbf{d})=\mathbf{0} \\
\mathbf{h}_{2}(\dot{\mathbf{x}}, \mathbf{x}, \mathbf{y}, \dot{\mathbf{u}}, \mathbf{u}, \mathbf{d}) \geq \mathbf{0}
\end{array}\right.
$$

in which $\mathbf{x} \in \boldsymbol{R}^{r}$ are the state variables of the process. Also, $\mathbf{u} \in \boldsymbol{R}^{m}, \mathbf{d} \in \boldsymbol{R}^{q}$, and $\mathbf{y} \in$ $\boldsymbol{R}^{n}$ are the vectors of the inputs, disturbances, and the output variables, respectively. The time derivatives of $\mathbf{x}$ and $\mathbf{u}$ are denoted by $\dot{\mathbf{x}}$ and $\mathbf{u}$, respectively, and $\mathbf{f}$ and $\mathbf{g}$ represent the nonlinear maps. The functions $\mathbf{h}_{\mathbf{1}}$ and $\mathbf{h}_{\mathbf{2}}$ include the process constraints, e.g., associated with production and safety specifications. In past operability approaches, the model structure $\mathbf{M}$ was defined by first-principles. In this thesis, this structure is also 
extended to include process simulator runs (for example in Aspen Plus $₫$ ) to obtain the relationships between input and output spaces. The manipulated inputs, u, can take values in the AIS, based on the design of the process that is limited by the process constraints (see Equation 3.2). Additionally, the disturbance variables, d, which can represent the process uncertainties such as ambient conditions, catalyst activity, or uncontrolled flow/composition of feed streams, present values inside the expected disturbance set (EDS) as defined in Equation 3.3. In this thesis, the disturbance variable, d, is considered equal to zero, $\mathbf{d}=0$, i.e., design operating point without disturbances. Based on the process model, the AOS is defined by the ranges of the outputs, $\mathbf{y}$, that can be achieved using the inputs inside the AIS as shown in Equation 3.4.

$$
\begin{gathered}
\text { AIS }=\left\{\mathbf{u} \mid \mathbf{u}_{i}^{\min } \leq \mathbf{u}_{i} \leq \mathbf{u}_{i}^{\max } ; 1 \leq i \leq m\right\} \\
\mathrm{EDS}=\left\{\mathbf{d} \mid \mathbf{d}_{i}^{\min } \leq \mathbf{d}_{i} \leq \mathbf{d}_{i}^{\max } ; 1 \leq i \leq q\right\} \\
\text { AOS }=\{\mathbf{y} \mid \mathbf{y}=\mathbf{M}(\mathbf{u}, \mathbf{d}) ; \mathbf{u} \in \text { AIS, } \mathbf{d} \text { is fixed }\}
\end{gathered}
$$

For some cases, the AOS does not satisfy desired production requirements, such as production rates or product qualities. These requirements associated with the outputs would comprise the DOS mathematically represented by Equation 3.5.

$$
\mathrm{DOS}=\left\{\mathbf{y} \mid \mathbf{y}_{j}^{\min } \leq \mathbf{y}_{j} \leq \mathbf{y}_{j}^{\max } ; 1 \leq j \leq n\right\}
$$

The DOS can be described by continuous edges that connect the vertices given by $\mathbf{y}^{\min }$ and $\mathbf{y}^{\max }$ for each output or discontinuous/disjoint points that discretize such edges. Considering that the DOS can be defined as the output space where the process should be operated, the set of inputs required to reach the entire DOS is defined as the DIS. In particular, if the DOS is a subset of the AOS, a calculation of the DIS for linear and linearized systems can be obtained by model inversion as shown in Equation 3.6. 


$$
\mathrm{DIS}=\left\{\mathbf{u} \mid \mathbf{u}=\mathbf{M}^{-\mathbf{1}} \mathbf{y} ; \mathbf{y} \in \mathrm{DOS}\right\}
$$

\subsection{DMA-MR Motivating Example}

\subsubsection{DMA-MR model}

To motivate the process operability approach and concepts, an example is first presented for the calculation of input-output spaces involving the DMA-MR system for the production of hydrogen and benzene. As briefly mentioned above, MRs are emerging systems that enable process intensification by combining two unit operations (reaction and separation) in one process, resulting in higher efficiencies and lower costs. In particular, in the DMA-MR process, higher methane conversion than in conventional packed-bed reactors is enabled due to the selective hydrogen removal from the reaction side through the membrane. This removal shifts the reaction equilibrium towards the products, thus increasing methane conversion and benzene production rate. A nonlinear operability analysis is performed here employing the DMA-MR system assuming a cocurrent reactor design configuration. Specifically, considering the shell and tube MR design in Figure 3.1, in which $\mathrm{CH}_{4}$ feeds the reaction side (tube packed with catalysts) and the sweep gas (e.g., helium) flows in the permeation side (shell), the $\mathrm{H}_{2}$ produced in the tube permeates to the shell through the membrane layer that is placed on the surface of the tube wall. The produced outlet streams from the tube (retentate) and shell (permeate) are rich in $\mathrm{C}_{6} \mathrm{H}_{6}$ and $\mathrm{H}_{2}$, respectively. 


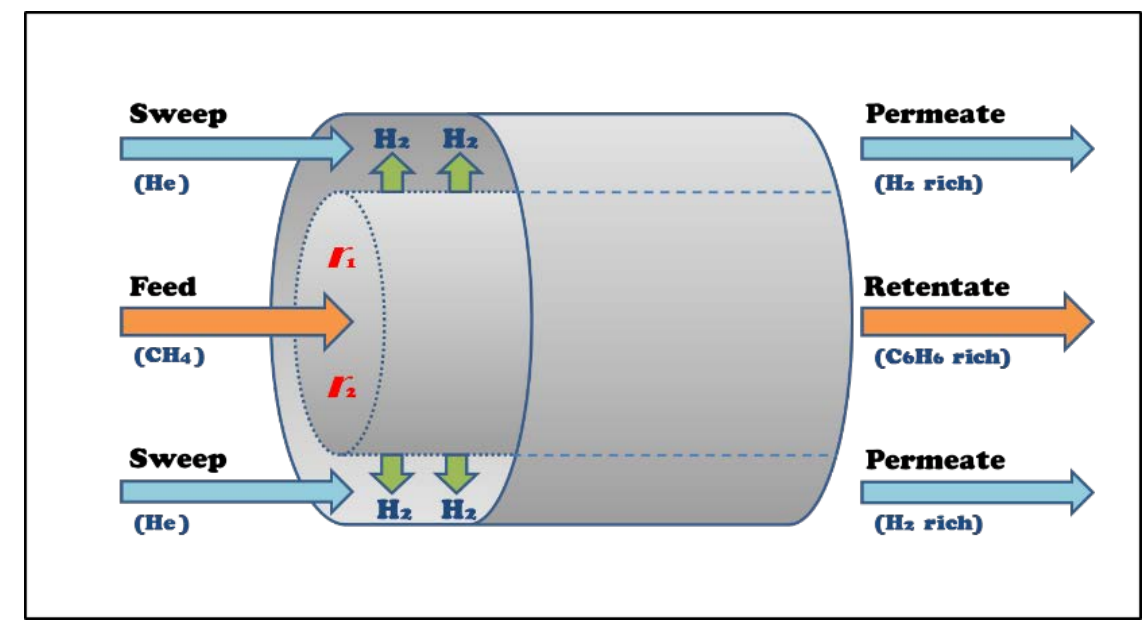

Figure 3.1. Schematic of DMA-MR with a cocurrent configuration

The following two-step reaction mechanism in gas phase (adapted from Li et al., 2002) is considered for this process:

Step 1:

$$
\begin{gathered}
2 \mathrm{CH}_{4}=\mathrm{C}_{2} \mathrm{H}_{4}+2 \mathrm{H}_{2} \\
r_{1}=k_{1} \mathrm{C}_{C H_{4}}\left(1-\frac{k_{1}^{\prime} \mathrm{C}_{C_{2} H_{4}} \mathrm{C}_{H_{2}}^{2}}{k_{1} \mathrm{C}_{C H_{4}}^{2}}\right)
\end{gathered}
$$

Step 2:

$$
\begin{gathered}
3 \mathrm{C}_{2} \mathrm{H}_{4}=\mathrm{C}_{6} \mathrm{H}_{6}+3 \mathrm{H}_{2} \\
r_{2}=k_{2} \mathrm{C}_{C_{2} H_{4}}\left(1-\frac{k_{2}^{\prime} \mathrm{C}_{C_{6} H_{6}} \mathrm{C}_{H_{2}}^{3}}{k_{2} \mathrm{C}_{C_{2} H_{4}}^{3}}\right)
\end{gathered}
$$

in which $r_{1}$ and $r_{2}$ represent the reaction rates of steps 1 and 2 , respectively, and $k_{1}, k_{1}^{\prime}$, $k_{2}$, and $k_{2}^{\prime}$ correspond to the reaction rate constants (where the symbol' denotes the inverse reaction). Also, $\mathrm{C}_{\mathrm{CH}_{4}}, \mathrm{C}_{\mathrm{C}_{2} \mathrm{H}_{4}}, \mathrm{C}_{\mathrm{H}_{2}}$, and $\mathrm{C}_{\mathrm{C}_{6} \mathrm{H}_{6}}$ are the concentrations of methane, ethylene, hydrogen, and benzene, respectively. Process operability calculations are 
performed by mapping the AIS to obtain the AOS using the nonlinear process model that describes the DMA-MR. For this purpose, the following mass balances are developed:

Molar balances inside tube:

$$
\begin{gathered}
\frac{d F_{t, C H_{4}}}{d z}=r_{1} A_{t}-\frac{Q}{\propto_{H_{2} / C H_{4}}}\left(P_{t, C H_{4}}^{1 / 4}-P_{s, C H_{4}}^{1 / 4}\right) \pi D_{t} \\
\frac{d F_{t, C_{2} H_{4}}}{d z}=-\frac{r_{1}}{2} A_{t}+r_{2} A_{t}-\frac{Q}{\propto_{H_{2} / C_{2} H_{4}}}\left(P_{t, C_{2} H_{4}}^{1 / 4}-P_{s, C_{2} H_{4}}^{1 / 4}\right) \pi D_{t} \\
\frac{d F_{t, H_{2}}}{d z}=-r_{1} A_{t}-r_{2} A_{t}-Q\left(P_{t, H_{2}}^{1 / 4}-P_{s, H_{2}}^{1 / 4}\right) \pi D_{t} \\
\frac{d F_{t, C_{6} H_{6}}}{d z}=-\frac{r_{1}}{3} A_{t}-\frac{Q}{\alpha_{H_{2} / C_{6} H_{6}}}\left(P_{t, C_{6} H_{6}}^{1 / 4}-P_{s, C_{6} H_{6}}^{1 / 4}\right) \pi D_{t}
\end{gathered}
$$

Molar balances inside shell:

$$
\begin{gathered}
\frac{d F_{s, C H_{4}}}{d z}=\frac{Q}{\propto_{H_{2} / C H_{4}}}\left(P_{t, C H_{4}}^{1 / 4}-P_{s, C H_{4}}^{1 / 4}\right) \pi D_{t} \\
\frac{d F_{s, C_{2} H_{4}}}{d z}=\frac{Q}{\propto_{H_{2} / C_{2} H_{4}}}\left(P_{t, C_{2} H_{4}}^{1 / 4}-P_{s, C_{2} H_{4}}^{1 / 4}\right) \pi D_{t} \\
\frac{d F_{s, H_{2}}}{d z}=Q\left(P_{t, H_{2}}^{1 / 4}-P_{s, H_{2}}^{1 / 4}\right) \pi D_{t} \\
\frac{d F_{s, C_{6} H_{6}}}{d z}=\frac{Q}{\propto_{H_{2} / C_{6} H_{6}}}\left(P_{t, C_{6} H_{6}}^{1 / 4}-P_{s, C_{6} H_{6}}^{1 / 4}\right) \pi D_{t}
\end{gathered}
$$

in which $Q$ is the $\mathrm{H}_{2}$ permeance through the membrane and $\propto_{H_{2} / c}$ is the selectivity between $\mathrm{H}_{2}$ and component $c$. Also, $P_{t, c}$ and $P_{s, c}$ are the partial pressures of components $c$ in the feed (tube) and permeate (shell) sides of the membrane, respectively. The membrane flux expression with a relationship proportional to the transmembrane partial pressure gradient of the species with a $1 / 4$ dependence is considered for an ion-based 
perovskite membrane, $\mathrm{SrCe}_{0.7} \mathrm{Zr}_{0.2} \mathrm{Eu}_{0.1 \mathrm{O}_{3-\delta}}$ (Li et al., 2011). $F_{t, c}$ and $F_{S, c}$ correspond to the molar flow rate of component $c\left(\mathrm{CH}_{4}, \mathrm{C}_{2} \mathrm{H}_{4}, \mathrm{H}_{2}\right.$ and $\left.\mathrm{C}_{6} \mathrm{H}_{6}\right)$ inside the tube and shell, respectively. Finally, $z, D_{t}$, and $A_{t}$ are the differential length, diameter and cross-sectional area of the reactor, respectively.

The simulation setup considers the following initial set of conditions for the reaction side: volumetric feed flow of $\mathrm{CH}_{4}$ of 8 [ $\mathrm{cm}^{3} / \mathrm{min}$ ], a total pressure of 1 [atm], and $k_{1}, k_{2}$, $k_{1}^{\prime}$, and $k_{2}^{\prime}$ values of $0.04\left[\mathrm{~s}^{-1}\right], 4.2\left[\mathrm{~s}^{-1}\right], 6.4 \times 10^{6}\left[\mathrm{~cm}^{3} / \mathrm{s} . \mathrm{mol}\right]$, and $56.38\left[\mathrm{~cm}^{3} / \mathrm{s} . \mathrm{mol}\right]$, respectively. Additionally, for the shell side, a flow rate of sweep gas (He) of 10 [cm $3 / \mathrm{min}]$, a total pressure of 1 [atm], and a diameter of $3[\mathrm{~cm}]$ are assumed. Isothermal reactor operation at a temperature of $900^{\circ} \mathrm{C}$ due to the presence of a furnace is also considered. Moreover, the reaction rate constant units from the original source (Li et al., 2002) have been converted to a reactor volume basis by using the catalyst bed density in the packed bed reactor. The ideal gas law is employed to calculate the concentrations that are needed to obtain the reaction rates. Finally, the mathematical model described by Equations (3.7-3.10) corresponds to a set of ordinary differential equations (ODEs) that can be solved using the MATLAB subroutine "ode15s". 


\subsubsection{DMA-MR operability analysis}

Case study $(2 \times 2)$

Here, two operability analyses for $2 \times 2$ systems (two inputs and two outputs) are carried out considering different sets of input variables. For the first case study, membrane selectivity and permeance are the inputs that define the AIS. Also, the production rate of benzene from the retentate and conversion of methane are the outputs that represent the AOS. The ranges considered for membrane permeance and selectivity are $0.0001-0.01\left[\mathrm{~mol} /\left(\mathrm{s} . \mathrm{m}^{2} . \mathrm{atm}^{1 / 4}\right)\right]$ and $200-100,000$, respectively. For the performed analyses, a grid is built by assuming that these input ranges are equally divided by 10 , which correspond to increments of $0.00099\left[\mathrm{~mol} /\left(\mathrm{s} \cdot \mathrm{m}^{2} \cdot \mathrm{atm}^{1 / 4}\right)\right]$ and 9,980 , for each variable, respectively. The tube length of $100[\mathrm{~cm}]$ and the inner tube diameter of $0.5[\mathrm{~cm}]$ are assumed as constant parameters. Figure 3.2 shows the AIS considered and the AOS
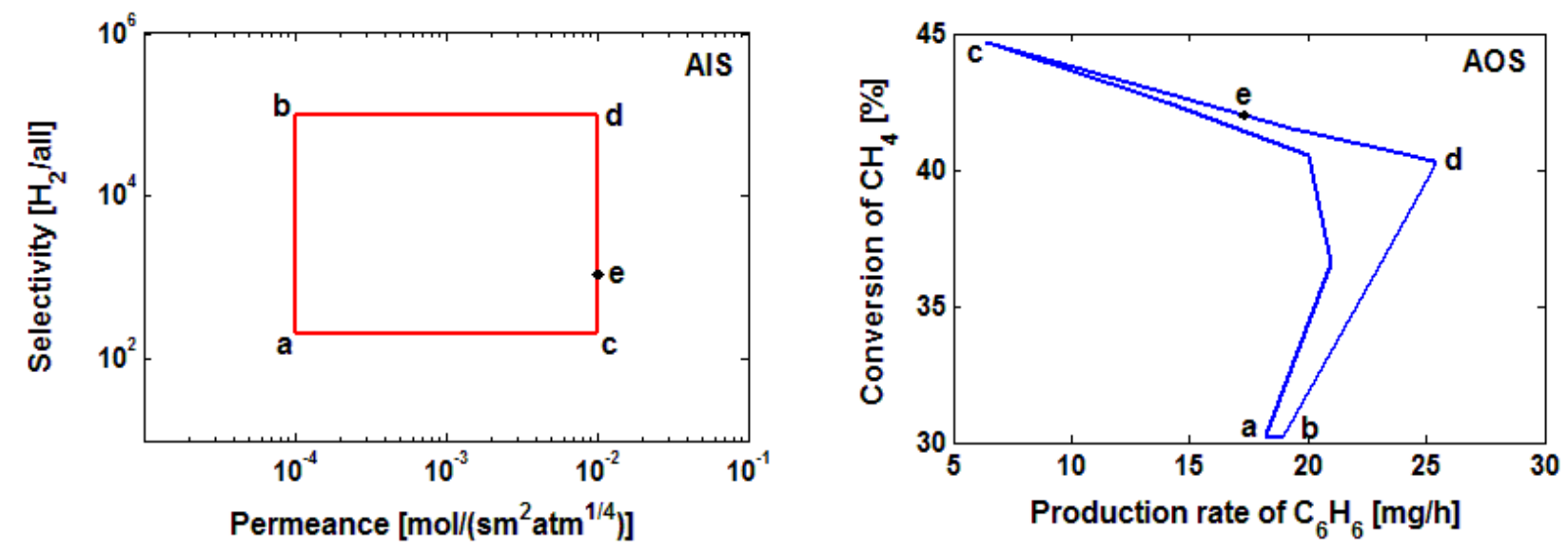

calculated for this case.

Figure 3.2. 2-D DMA-MR system. Input variables: membrane permeance and selectivity (AIS on the left).

Process outputs: conversion of methane and production rate of benzene (AOS on the right) 
For the segment from points "a" to "b", which shows a constant low permeance value $\left(10^{-4}\left[\mathrm{~mol} /\left(\mathrm{s} \cdot \mathrm{m}^{2} \cdot \mathrm{atm}^{1 / 4}\right)\right]\right)$ for gradually increasing selectivities (from 200 to $10^{5}$ ), only slight changes in methane conversion and benzene production rate are obtained. When the permeance is increased to $0.01\left[\mathrm{~mol} /\left(\mathrm{s} \cdot \mathrm{m}^{2} \cdot \mathrm{atm}^{1 / 4}\right)\right]$, from points "b" to "d", both production rate and conversion present positive changes. Particularly, the vertex "d" in the AIS figure is associated with the highest values of membrane permeance and selectivity, $0.01\left[\mathrm{~mol} /\left(\mathrm{s} . \mathrm{m}^{2} \cdot \mathrm{atm}^{1 / 4}\right)\right]$ and $10^{5}$, respectively. This point corresponds to the best production rate of benzene $(\approx 26[\mathrm{mg} / \mathrm{h}])$. However, the highest conversion of methane (around 45\%) occurs at point " $\mathrm{c}$ ", where the loss of benzene through the membrane is also the highest, due to the low selectivity value at that point, shifting the equilibrium of reaction step 2 even further. This result indicates that increasing the conversion of methane does not necessarily imply having a higher production of the desired benzene product.

The performed operability analysis suggests that: (i) the permeance parameter is more critical for obtaining a high reaction conversion when compared to the selectivity; and (ii) for a high permeance, a sufficiently high selectivity value is also needed for optimal production rates. Finally, point "e" represents a case with membrane properties that would be expected in the lab, such as a permeance of $0.01\left[\mathrm{~mol} /\left(\mathrm{s} \cdot \mathrm{m}^{2} \cdot \mathrm{atm}^{1 / 4}\right)\right]$ and a selectivity of 1000 . For this point, the production rate of benzene is about $17[\mathrm{mg} / \mathrm{h}]$ and the methane conversion is approximately $42 \%$. Also for a $2 \times 2$ system, new input variables are now considered as the tube diameter and length, keeping the production rate of benzene and conversion of methane as the output variables. Figure 3.3 shows the plots of the input 
and output spaces for this case. In this analysis, the membrane permeance and selectivity are fixed at the point "e" from the previous case. The range of simulation for the tube diameter and length are $0.5-2[\mathrm{~cm}]$ and $10-100[\mathrm{~cm}]$, respectively.
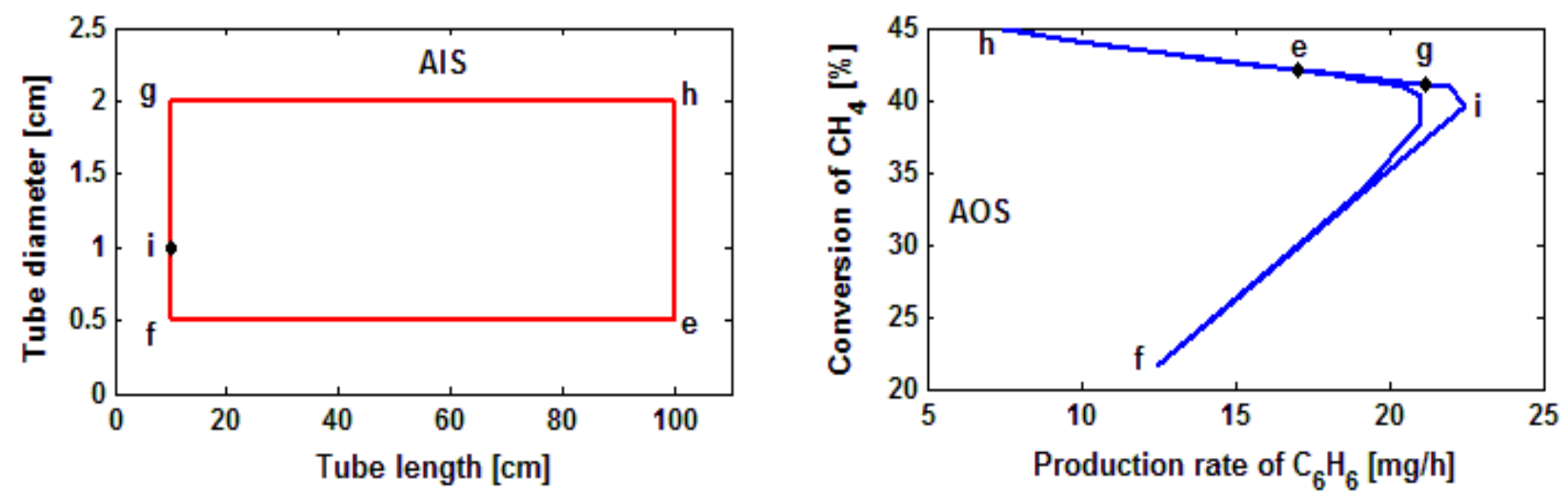

Figure 3.3. 2-D DMA-MR system. Input variables: diameter and length of tube (AIS on the left).

Process outputs: conversion of methane and production rate of benzene (AOS on the right)

According to Figure 3.3, the points with the highest length of 100 [cm], "e" and "h", reach the highest conversion of methane ( $\approx 42$ and $45 \%$, respectively). For such points, as the diameter decreases from 2 to $0.5[\mathrm{~cm}]$, the benzene flow in the retentate increases from approximately 7 to 17 [mg/h]. This increase happens due to the smaller available area for permeation of benzene through the membrane. The point " $\mathrm{i}$ " presents the best production of benzene $(\approx 22[\mathrm{mg} / \mathrm{h}])$ that occurs when the diameter and length of tube are $1[\mathrm{~cm}]$ and $10[\mathrm{~cm}]$, respectively. In this case, the recommendation from the traditional operability studies would be to follow the path from "f" to " $g$ ", specifically the point "i", where similar performance is achieved when compared to point "e" for a much smaller reactor size (reduction of $\approx 60 \%$ and $80 \%$ in reactor volume and membrane area, respectively). 


\section{Case study $(3 \times 3)$}

With the purpose of increasing the dimensionality of the operability approach for process design of the DMA-MR system, now a $3 \times 3$ system is analyzed, in which the membrane selectivity, tube length and diameter are the input variables that define the AIS. For this case, the membrane permeance is fixed at $0.01\left[\mathrm{~mol} /\left(\mathrm{s} \cdot \mathrm{m}^{2} \cdot \mathrm{atm}^{1 / 4}\right)\right]$. The selected range of simulation for the membrane selectivity, the tube diameter and length are $100-100,000,0.5-2[\mathrm{~cm}]$ and $10-100[\mathrm{~cm}]$, respectively. For the performed operability analysis, a grid of 1000 points for the input variables is built by assuming that their ranges are equally divided by 10 . The output variables that define the AOS correspond here to the production rates of benzene and hydrogen, as well as the methane conversion. Figure 3.4 shows the AIS (parallelepiped represented by solid red dots) considered and the calculated AOS in this case (solid blue dots). The plot of the calculated AOS (Figure 3.4 on the right) depicts three zones that are worth analyzing. In particular, zone "b" represents the best balance between benzene and hydrogen production rates with high methane conversions. According to the operability analysis, this zone is obtained when the membrane selectivity is the highest, 100,000 , for moderate reactor volumes. For such volumes, the selected reactor diameter and length present an inverse behavior, i.e., high length for a low diameter, or high diameter for a low length value. For instance, for the mentioned membrane selectivity, and tube length and diameter of 100 [cm] and $0.5[\mathrm{~cm}]$, respectively, it is possible to obtain production rates of benzene and hydrogen, and methane conversion of approx. $25.30[\mathrm{mg} / \mathrm{h}], 3.90[\mathrm{mg} / \mathrm{h}]$, and $40.29[\%]$, respectively, for a catalytic MR volume of $19.6\left[\mathrm{~cm}^{3}\right]$. Also in this zone, for a tube length 
and diameter of $10[\mathrm{~cm}]$ and $2[\mathrm{~cm}]$, respectively, it is possible to obtain production rates of benzene and hydrogen, and methane conversion of about $25.40[\mathrm{mg} / \mathrm{h}], 3.89[\mathrm{mg} / \mathrm{h}]$, and 40.27 [\%], respectively, with a reactor volume of $31.4\left[\mathrm{~cm}^{3}\right]$. The obtained moderate membrane surface areas, associated with these moderate reactor volumes, and high selectivity are needed to preclude the permeation of benzene through the membrane.
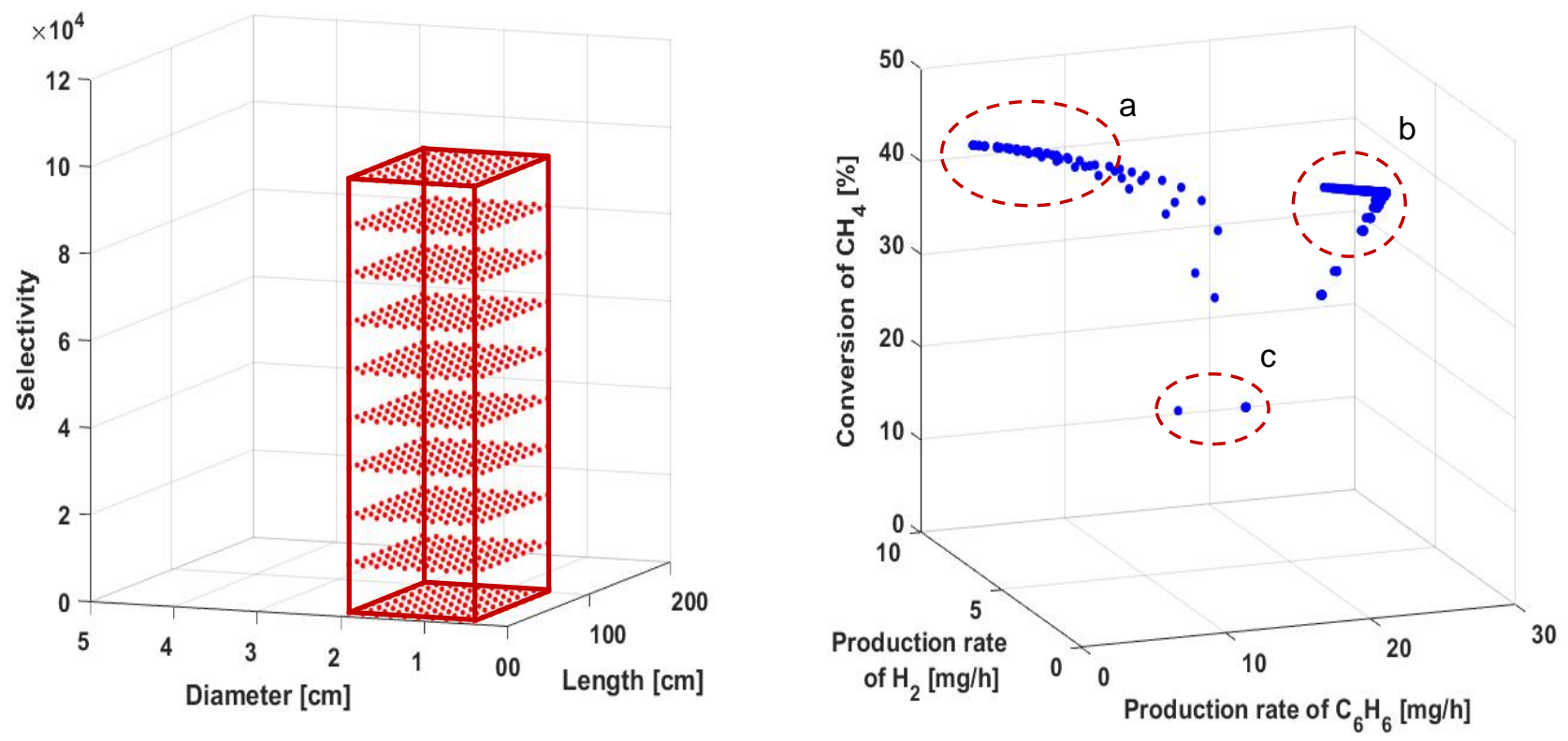

Figure 3.4. 3-D DMA-MR system. Input variables: reactor diameter, length, and membrane selectivity (AIS on the left). Process outputs: conversion of methane, production rates of benzene and hydrogen (AOS on the right)

In contrast, zone "a" shows low process production rates caused mainly by the low membrane selectivity and the high reactor length and diameter values. For example, when the membrane selectivity is 100 , and the tube length and diameter are $100[\mathrm{~cm}]$ and $2[\mathrm{~cm}]$, respectively, the results show an undesired performance with the benzene production rate of almost zero $[\mathrm{mg} / \mathrm{h}]$, even though the methane conversion is $\approx 45.76$ 
[\%]. In particular, the membrane low selectivity and high surface area result in a high permeation of benzene through the membrane. This outcome indicates once again that high methane conversions do not translate to high benzene production rates. Finally, zone "c" is mainly characterized by the lowest values of the tube length and diameter, such as $10[\mathrm{~cm}]$ and $0.5[\mathrm{~cm}]$, respectively. The short residence times and reduced membrane surface areas for this MR design cause a low methane conversion and a limited reaction equilibrium shifts towards products due to low hydrogen permeation. In order to include desired output requirements in the operability analysis, for instance specific ranges for methane conversion and production rates of benzene and hydrogen, a desired output set should be considered. With this last consideration, two scenarios arise for the operability analysis. If the DOS is a subset of the AOS, the calculated DIS can be obtained by model inversion, as showed in Equation (3.6). Now if the DOS is not a subset of the AOS, scenario that would probably be more likely to occur in practice, a systematic approach needs to be developed to calculated the feasible DIS (DIS*). In the next chapter, a detailed operability framework for nonlinear systems is presented employing multi-model and optimization approaches, when the conventional calculation of the DIS (in Equation 3.6) cannot be employed. 


\section{Chapter 4 Novel Operability Approaches for Process Design}

The AOS calculations presented in the previous chapter can provide an insight to the user on how to select the DOS. For instance, if the user would like to push a certain performance variable value towards more desired values, this factor could be included in the specification of the corresponding DOS dimension. When the DOS is a subset of the AOS, a multi-model approach is first presented in this chapter to determine the DIS, using linearized versions of the process model at different operating regions. The AOS may not completely satisfy desired production requirements, environmental constraints, and target specifications within the DOS. In this case, alternative paths to calculate the feasible DIS, or DIS*, should be developed. In this chapter, an optimization-based operability algorithm is also formulated to calculate the DIS* from the DOS.

A schematic diagram of the proposed operability framework for process design is presented in the Figure 4.1, in which both multi-model and optimization-based operability approaches are summarized. In this figure, the traditional operability approach applied to determine the AOS from the AIS is represented by the continuous black lines. The multimodel approach is depicted by the dotted green lines, and the optimization-based operability approach is indicated by the dashed red lines. The steps of this novel operability framework will be detailed below. 


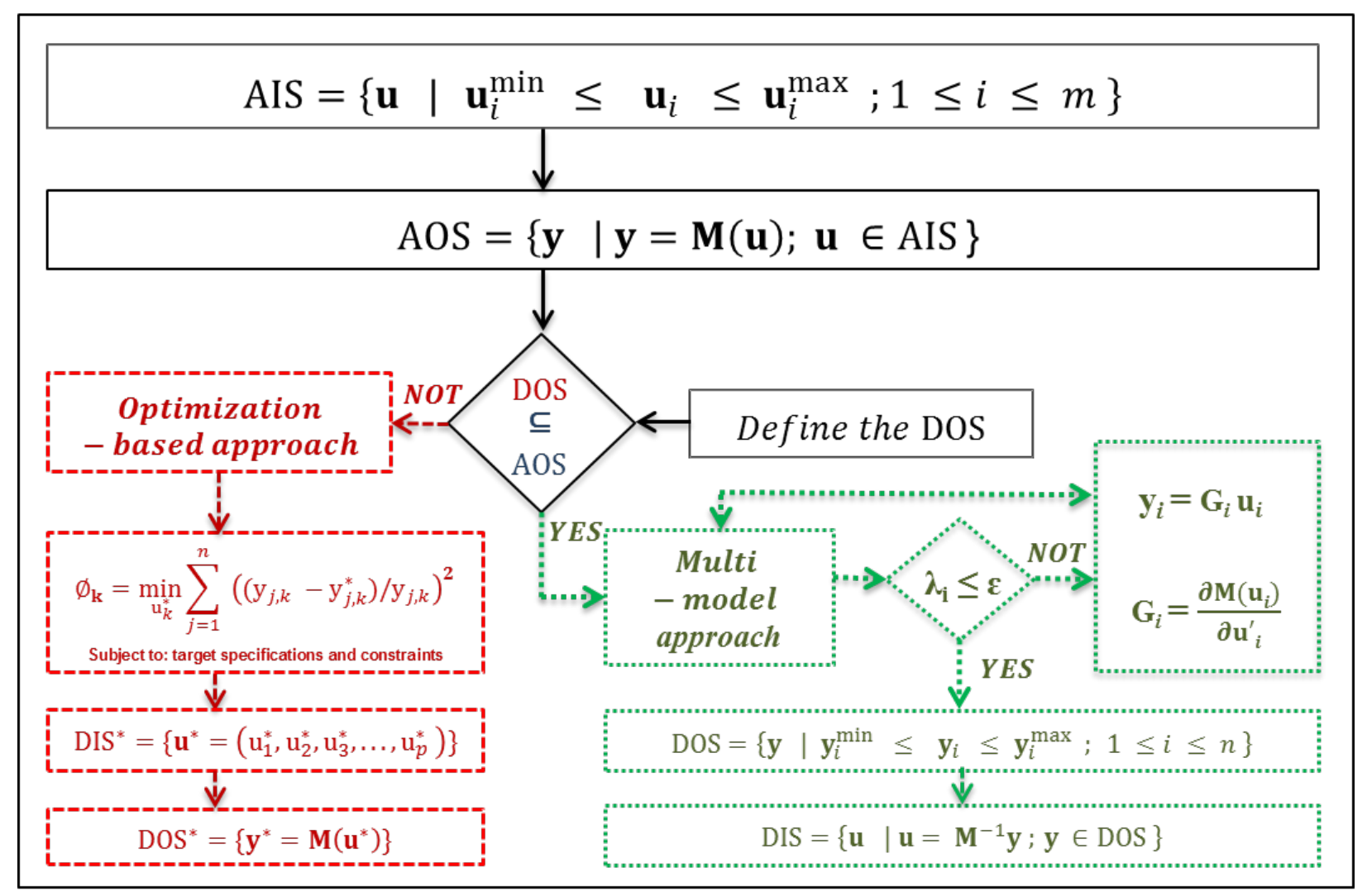

Figure 4.1. Schematic of operability framework for process design

\subsection{Multi-Model Approach}

When the AOS satisfy the requested DOS, the DIS can be calculated by model inversion (Equation 4.1) using the multi-model approach. In this case, the selected DOS is divided in different operating regions, so that linearized versions of the process model can be obtained to allow the calculation of the DIS for such regions.

$$
\text { DIS }=\left\{\mathbf{u} \mid \mathbf{u}=\mathbf{M}^{-\mathbf{1}} \mathbf{y} ; \mathbf{y} \in \text { DOS }\right\}
$$




\subsubsection{Problem formulation}

The first step to calculate the DIS consists of identifying the different operating regimes for the process of interest. For such identification, an algorithm has been developed on the basis of the properties of each individual linear system collected from linearizations of the original nonlinear model. Particularly, for each operating region $i$, the following model description is considered:

$$
\mathbf{y}_{i}=\mathbf{G}_{i} \mathbf{u}_{\mathrm{i}} ; \mathbf{u}_{\mathrm{i}} \in \text { AIS }
$$

in which the intersection of all possible values of $\mathbf{y}_{i}$ defines the AOS.

For each subset $i$ of the given AIS, the process gain matrix, $\mathbf{G}_{i}$, is computed by linearization of the process model, $\mathbf{M}$, through sensitivity calculations as follows:

$$
\mathbf{G}_{i}=\frac{\partial \mathbf{M}\left(\mathbf{u}_{\mathbf{i}}\right)}{\partial \mathbf{u}_{i}^{\prime}}
$$

Then, the properties of each linearized model, such as the eigenvalues of $\mathbf{G}_{i}, \lambda_{i}$, are analyzed to define how many different individual models are needed to represent the process operating points of the original nonlinear system. In case there are similarities between different models, one model, for instance, is chosen to represent more than one operating point. This procedure allows obtaining the minimum number of models that is necessary to adequately describe the process operating envelope. The calculation of the DIS from the DOS is then carried out for each linearized model using Equation 4.1. To demonstrate this multi-model approach, in the next example, the DMA-MR $2 \times 2$ system 
from Chapter 3 is revisited, and the DIS is calculated from a selected DOS that is a subset of the AOS.

\subsubsection{DMA-MR case study}

Figure 4.2 shows the initial AIS and the computed AOS (solid red and blue lines, respectively), including the desired output set (dotted green line). In this case, the calculations follow the $2 \times 2$ motivating example presented in the previous chapter. Specifically, the available input set for the DMA-MR is described by the reactor tube diameter and length, from 0.5 to $2[\mathrm{~cm}]$ and 10 to $100[\mathrm{~cm}]$, respectively, and the selected DOS corresponds to benzene production rate and methane conversion in the following ranges, 15 to $25[\mathrm{mg} / \mathrm{h}]$ and 35 to $45[\%]$, respectively.
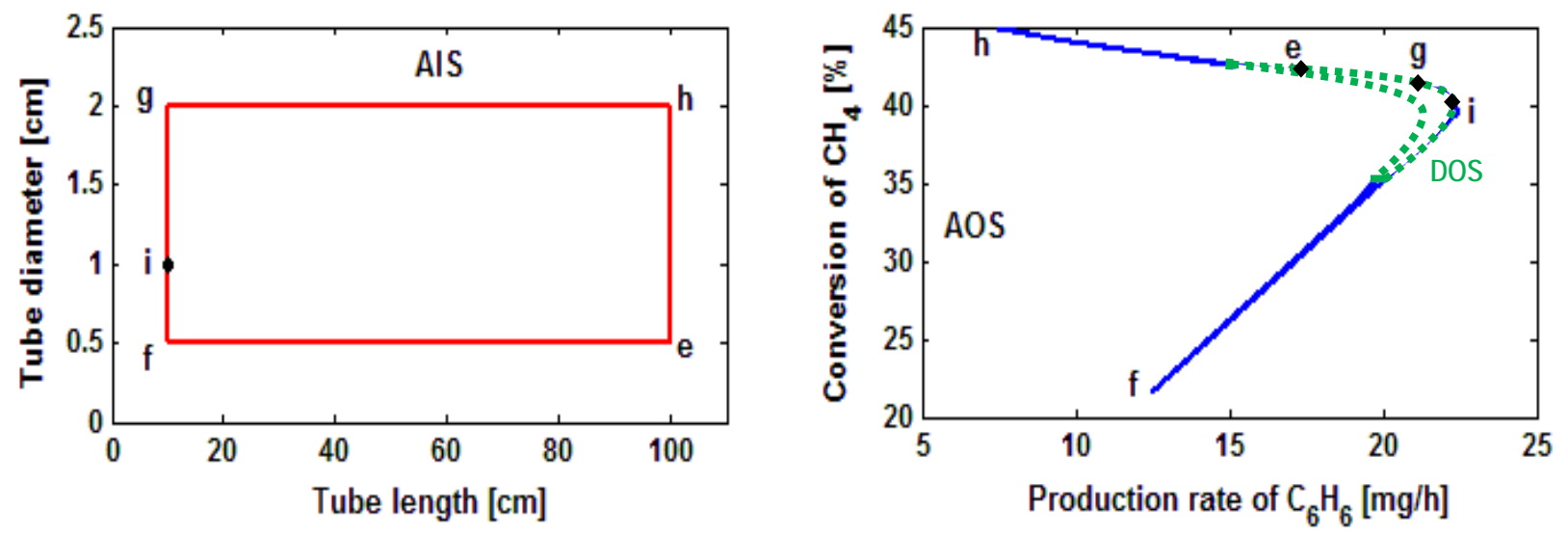

Figure 4.2. 2-D DMA-MR system. Input variables: diameter and length of tube (AIS on the left). Process outputs: conversion of methane and production rate of benzene (AOS and DOS on the right)

Using the expressions described by Equations 4.2 and 4.3, it is possible to obtain linearized versions of the process model at different operating regions that define the 
intersection of the AOS (solid blue line) with the DOS (dotted green line). The process gain matrix used is $2 \times 2$ (inputs: reactor tube diameter and length, and outputs: benzene production rate and methane conversion), and 40 operating regions are defined. Through the sensitivity calculations in Equation 4.3, the eigenvalues as properties of each linearized model are calculated. A relative error $(\varepsilon)$ of $0.01 \%$ between eigenvalues for different models is set for the model selection. Considering this error for the multi-model operability approach, it is possible to obtain a reduction in the amount of models used by $50 \%$ (from 40 to 20 models). Figure 4.3 depicts, on the left, the output set (intersection between AOS and DOS) with output ranges for benzene production rate and methane conversion between 15 to $22[\mathrm{mg} / \mathrm{h}$ ] and 35 to 43 [\%], respectively. Using the inverse model expression in Equation 4.1, the DIS can be obtained from the DOS, where the process model matrix for each model is represented by the process gain matrix obtained by sensitivity calculations (see Figure 4.3 , on the right). Note that the DIS is a subset of the initial AIS (see Figure 4.2, on the left) as expected, as the selected DOS is part of the AOS.

The point " $\mathrm{i}$ " defined previously as part of the intersection between the AOS and the DOS presents the best production of benzene $(\approx 22[\mathrm{mg} / \mathrm{h}])$. It occurs when the diameter and length of tube are $1[\mathrm{~cm}]$ and $10[\mathrm{~cm}]$, respectively. Comparing this point "i" with point "e" as starting point (Figure 4.2, on the right), the tube diameter and length change from 0.5 to $1[\mathrm{~cm}]$ and 100 to $10[\mathrm{~cm}]$. Therefore, once again, the design of the membrane reactor can be reduced by $60 \%$ in volume (catalyst zone), and roughly $80 \%$ in membrane area when comparing both cases. These results show that after the DIS is 
determined by linearizations, it is possible to calculate an optimized process design using the derived linear models. The multi-model approach can thus be used as a tool for effective calculations of the DIS from a selected DOS within the AOS.
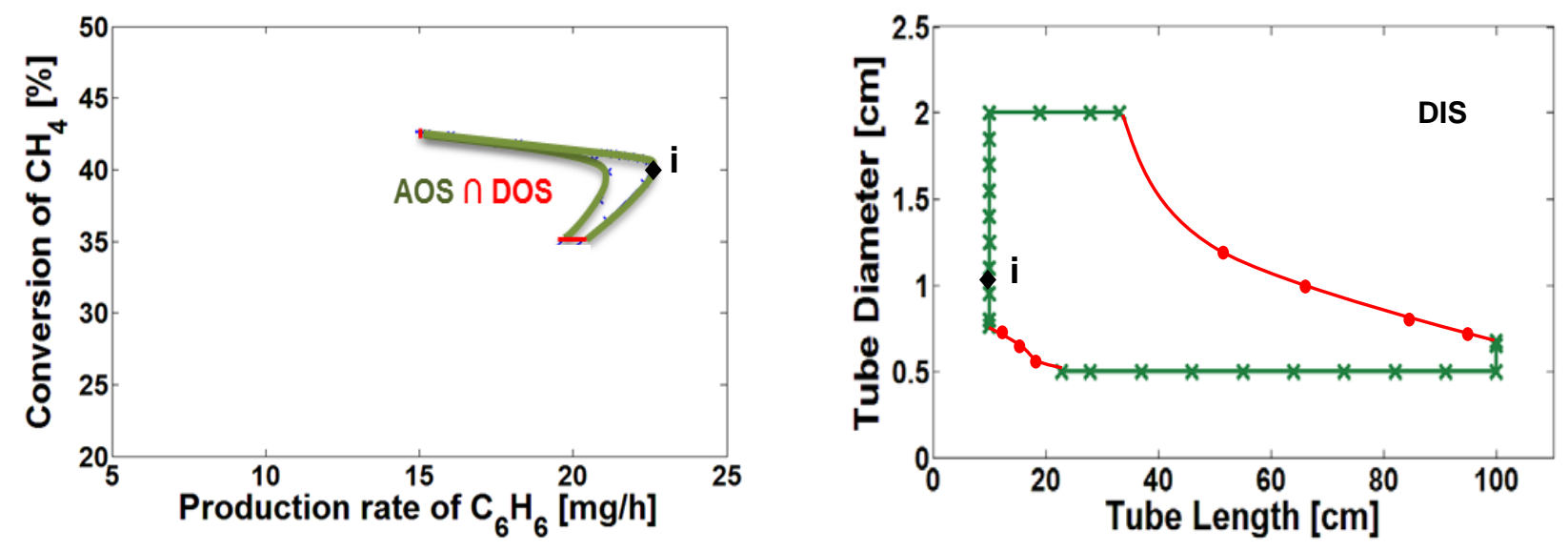

Figure 4.3. 3-D DMA-MR system. AOS intersection with DOS (on the left). Calculated DIS (on the right)

For the cases when the DOS is not completely contained in the AOS, an optimization-based approach will be introduced for operability calculations in the next subsection.

\subsection{Optimization-Based Operability Approach}

An optimization-based operability algorithm is formulated here for the calculation of the DIS for the general cases when the AOS does not contain the entire DOS. By 
employing nonlinear optimization approaches with constraints, this algorithm provides the feasible operating envelope for the DIS* given the constraints specified by the DOS.

\subsubsection{Problem formulation}

To calculate the feasible DIS from the DOS, an algorithm is developed by employing nonlinear programming (NLP) tools to minimize the objective function $\varnothing$ below. This function is mathematically defined by the relative error (or distance) between the desired output points from the DOS $\left(\mathrm{y}_{j, k}\right)$ and the feasible output points $\left(\mathrm{y}_{j, k}^{*}\right)$ as follows:

$$
\mathrm{P} 1: \quad \emptyset_{\mathrm{k}}=\min _{\mathrm{u}_{k}^{*}} \sum_{j=1}^{n}\left(\left(\mathrm{y}_{j, k}-\mathrm{y}_{j, k}^{*}\right) / \mathrm{y}_{j, k}\right)^{2}
$$

subject to: nonlinear model

$$
\begin{aligned}
& \mathrm{u}_{k}^{\min } \leq \mathrm{u}_{k}^{*} \leq \mathrm{u}_{k}^{\max } \\
& \mathbf{c}_{1}\left(\mathrm{u}_{k}^{*}\right) \leq \mathbf{0}
\end{aligned}
$$

in which $\mathrm{y}_{j, k}$ corresponds to the DOS selected points according to a specified grid. The desired output points are elements of the output set, $\mathrm{y}_{k}=\left(\mathrm{y}_{1, k}, \mathrm{y}_{2, k} \ldots \mathrm{y}_{j, k} \ldots \mathrm{y}_{n, k}\right)$. The subscript $j=1,2,3 \ldots . n$ represents the output variable index, and $k=1,2,3 \ldots p$ is defined as the point index for the total points in the grid. Considering this grid, the DOS can be written as

$$
\operatorname{DOS}=\left\{\mathbf{y} \mid \mathbf{y}=\left(\mathrm{y}_{1}, \mathrm{y}_{2}, \mathrm{y}_{3} \ldots \mathrm{y}_{p}\right)\right\}
$$


The output from the optimizer consists of the input set calculated for each point, $\mathrm{u}_{k}^{*}=\left(\mathrm{u}_{1, k}^{*}, \mathrm{u}_{2, k}^{*} \ldots \mathrm{u}_{i, k}^{*} \ldots \mathrm{u}_{m, k}^{*}\right)$. The subscript $i=1,2,3 \ldots m$ denotes the input variable index. Also $\mathbf{c}_{1}$ represents the nonlinear constraint map associated with specified target specifications or design conditions. The points in $\mathrm{u}_{k}^{*}$ define the DIS* as the feasible desired input set when the DOS is not a subset of the AOS. The DIS* is thus given by

$$
\operatorname{DIS}^{*}=\left\{\mathbf{u}^{*} \mid \mathbf{u}^{*}=\left(\mathrm{u}_{1}^{*}, \mathrm{u}_{2}^{*}, \mathrm{u}_{3}^{*} \ldots \mathrm{u}_{p}^{*}\right)\right\}
$$

This new input set can now be used to calculate the feasible DOS, DOS*, which is the closest vector set in terms of distance to each point of the DOS according to the objective function defined in P1. The DOS* calculated using the DIS* can be represented by

$$
\mathrm{DOS}^{*}=\left\{\mathbf{y}^{*} \mid \mathbf{y}^{*}=\mathbf{M}\left(\mathbf{u}^{*}\right) ; \mathbf{u}^{*} \in \mathrm{DIS}^{*}\right\}
$$

In particular, the process model $\mathbf{M}$ (for example in the DMA-MR given by Equations 3.9 and 3.10) is applied to the input points that describe the DIS* as defined in Equation 4.5. Additionally, note that the proposed method does not require first-principles models as in the traditional operability framework. As an alternative, simulator runs could be performed to obtain the relationship between the output points and the required inputs to achieve the desired outputs.

The DOS* can also be obtained directly as a result of the optimization problem P1, by storing the feasible output points that correspond to the optimal input points calculated by the optimizer. An initial guess input point $\mathrm{u}_{k, o}^{*}$ that is within the AIS ranges is requested 
to start the evaluation of the objective function. Also, the optimization problem P1 is characterized by a nonlinear objective function, with nonlinear constraints, which can be solved by the NLP solvers available in the Optimization toolbox in MATLAB ("fminsearch" or equivalent). In particular, "fminsearch" in MATLAB was successfully employed in the performed studies in this thesis.

To demonstrate the application of this problem P1, the following subsection will show case studies with selected DOS that are not subset of the AOS. The revisited case studies correspond to the DMA-MR 2x2 and 3x3 systems presented in Chapter 3.

\subsubsection{DMA-MR case study}

As the first step in the effort to demonstrate the novel optimization-based operability approach for design of processes described by nonlinear models, a lowdimensional MR example is considered in this subsection. In particular, the proposed optimization approach is applied to calculate the DIS considering the DOS presented in Figure 4.4 corresponding to the motivating $2 \times 2$ example above. The AIS and the DOS are defined by the reactor length $\left(\mathrm{u}_{1}\right)$ and diameter $\left(\mathrm{u}_{2}\right)$, and the benzene production rate $\left(\mathrm{y}_{1}\right)$ and methane conversion $\left(\mathrm{y}_{2}\right)$, respectively.

For this example, the original DOS is discretized considering $p=100$ points (i.e., the domain of the benzene production rate and methane conversion are divided in a $10 \times 10$ grid), thus $k=1,2,3 \ldots 100$. For the DIS* calculation, design specifications associated 
with the plug flow reactor assumption (Rawlings and Ekerdt, 2002), such as length over diameter, $L / D_{t} \geq 30$, and the reactor length for an experimental setup less than $300[\mathrm{~cm}]$, are incorporated into the objective function constraints. Also, positivity constraints on both the length and diameter are imposed.
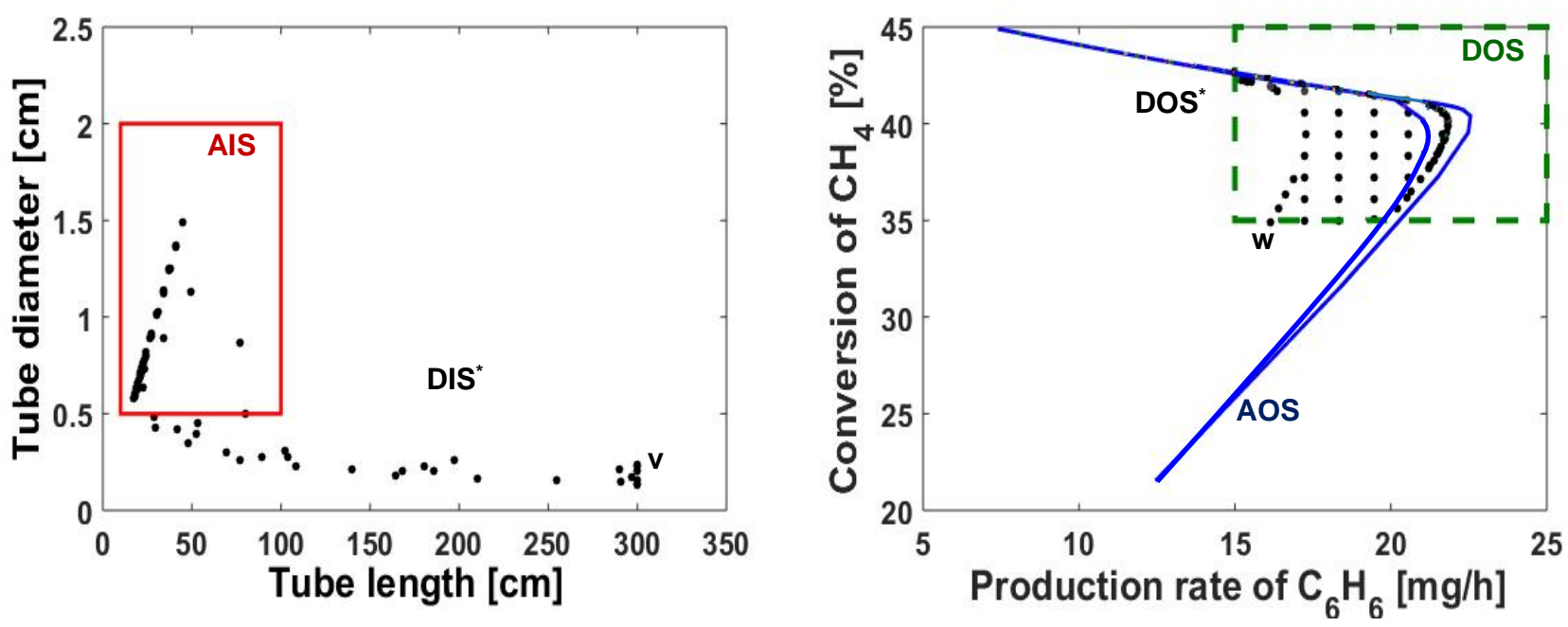

Figure 4.4. 3-D DMA-MR system. DIS* and AIS (on the left). DOS*, DOS and AOS (on the right)

When evaluating P1, as an example, for the bottom-left vertex in the DOS of Figure 4.4 (dashed green rectangle), i.e., $y_{1}=\left(y_{1,1}, y_{2,1}\right)=(15,35)$, and considering an initial guess for the inputs of $u_{1, o}^{*}=(10,0.5)$, which is the bottom-left vertex in the AIS of Figure 4.4 (red rectangle), the calculated feasible input point obtained is $u_{1}^{*}=\left(u_{1,1}^{*}, u_{2,1}^{*}\right)=$ (299.99, 0.13). This point, denoted as " $\mathrm{v",}$, is not part of the original AIS as depicted in Figure 4.4 (on the left). Applying the DMA-MR process model in Equations 3.9 and 3.10 to this point, the feasible output point obtained is $y_{1}^{*}=\mathbf{M}\left(u_{1}^{*}\right)=\left(y_{1,1}^{*}, y_{2,1}^{*}\right)=(16.03$, 35.00). This point is showed as "w" in the output space in Figure 4.4 (on the right), which 
corresponds to the closest point in distance to the bottom-left vertex of the DOS. For this and other case studies performed in this thesis, other initial guesses for the inputs have also been attempted, providing similar outcomes for the solved optimization problems.

The optimization problem is then solved for all of the DOS points in the established grid. The DIS* outcome from the optimizer for all such points is shown in Figure 4.4 (see solid black dots on the left). The calculated input points represent a region of approximate ranges for the length and diameter between $17-300[\mathrm{~cm}]$ and $0.12-1.60[\mathrm{~cm}]$, respectively. Note that this feasible input set obtained is not completely contained in the initial AIS (see red rectangle, Figure 4.4 on the left). The computed DOS* (see solid black dots, Figure 4.4 on the right) is a subset of the original DOS (see dashed green rectangle in the same figure) with ranges of the benzene production and methane conversion reduced to about $15-21.5[\mathrm{mg} / \mathrm{h}]$ and $35-42.5[\%]$, respectively. Finally, note that the DOS* is not a subset of the AOS (see solid blue lines, Figure 4.4 on the right) as expected because the initial AIS does not contain all the points of the DIS*.

These results show that the novel operability-based approach allows the calculation of the feasible input spaces for nonlinear systems. In particular, the space DIS* is comprised of the input points that satisfy as close as possible the specified target specifications and constraints in the original DOS. Using the information provided by the optimizer, the user could identify the necessary modifications to the reactor design that should be performed in order to achieve the feasible targets. 
In order to increase the number of desired output requirements in the DMA-MR analysis, the $3 \times 3$ case study presented in Chapter 3 is revisited. The DOS is now represented by a parallelepiped (Figure 4.5 on the left) for the selected benzene production rate from the retentate, hydrogen production rate from the permeate, as well as methane conversion in the ranges of $15-25[\mathrm{mg} / \mathrm{h}], 3-6[\mathrm{mg} / \mathrm{h}]$, and $35-45[\%]$, respectively.
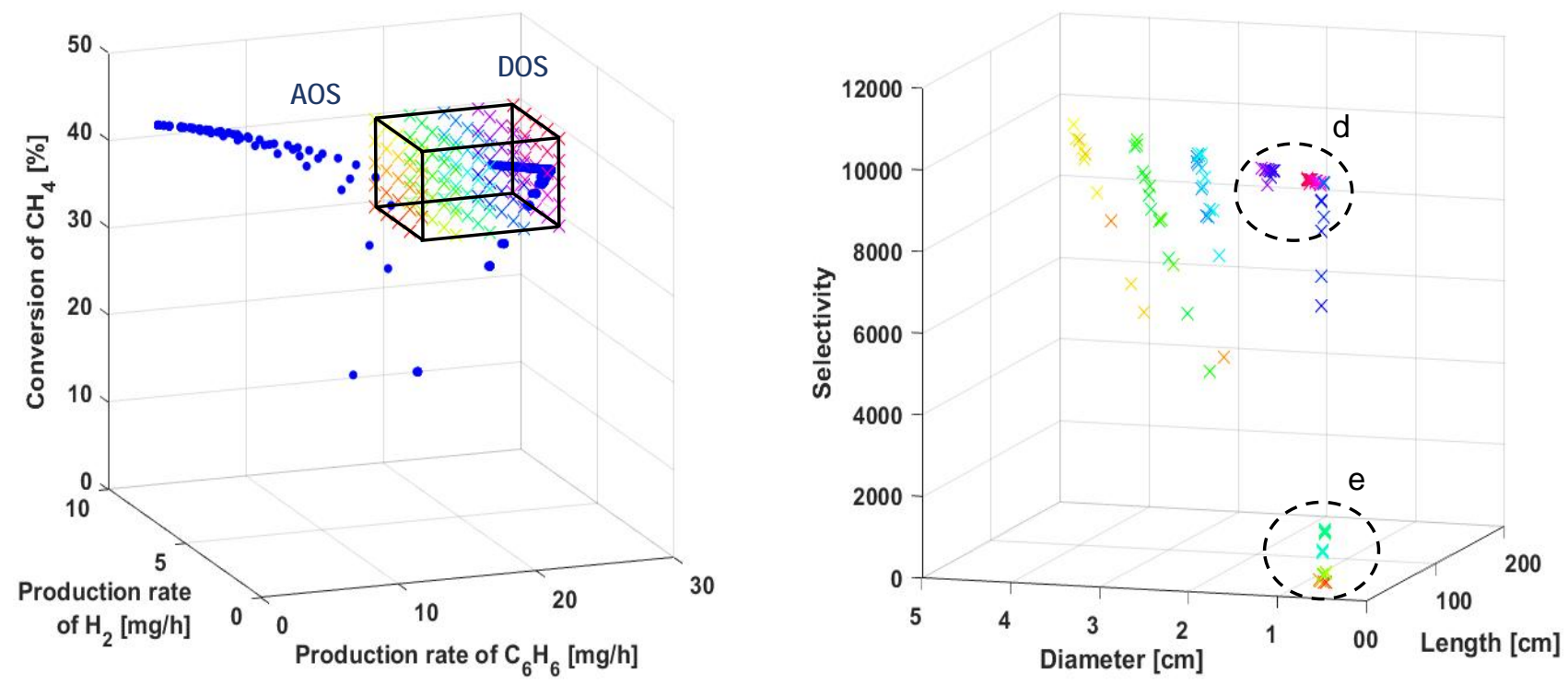

Figure 4.5. 3-D DMA-MR system. AOS and DOS (on the left). DIS* (on the right)

This DOS is selected around zone "b" in Figure 3.4 (Chapter 3), as this zone showed a good balance among the selected desired variables. The AOS is also presented in Figure 4.5 (see solid blue dots on the left) to provide a visual comparison between the currently achievable and the desired sets. It would be worth checking whether different points in the DOS could be possibly achievable after performing design changes reflected in the input space. 
To provide the feasible operating envelope for the DIS*, the operability-based algorithm defined in P1 is employed. For this example, the DOS is discretized considering $p=125$ points (i.e., the domains for the benzene and hydrogen production rates, as well as methane conversion are divided by 5 , which corresponds to increments of 2.5 [mg/h], $0.75[\mathrm{mg} / \mathrm{h}]$, and $2.5[\%]$, for each variable, respectively), thus $k=1,2,3 \ldots 125$ (see " $\mathrm{x}$ " symbols in Figure 4.5 on the left). The optimization problem P1 is solved for all of the DOS points in the established grid. For the solution of $\mathrm{P} 1$, positivity constraints are imposed on the input variables: reactor length, diameter, and membrane selectivity. Design specifications are also incorporated as process constraints, such as length over diameter $\geq 30$ for plug flow, reactor length for an experimental setup less than 300 [cm], and the membrane selectivity values closer to lab expectations of less than 10,000 .

The calculated DIS* that satisfies the process model, target specifications and constraints is shown in Figure 4.5 (on the right). The calculated input points (color coded with their respective DOS points) form a region for the reactor length and diameter, and membrane selectivity between $17.40-128.82$ [cm], $0.57-4.29$ [cm], and $208-10,000$, respectively. These results indicate that the feasible input set obtained is not completely contained in the initial AIS (Figure 3.4 on the left). The calculated DIS* has two zones that are worth analyzing, "d" and "e". Particularly, zones "d" and "e" show different benzene production rates between 20 and $25[\mathrm{mg} / \mathrm{h}]$, and 15 and 20 [mg/h], respectively, due to the extreme values of selectivity employed for each zone. 
These DMA-MR results applying problem P1 suggest that: i) this new optimizationbased operability approach can calculate the feasible input spaces for nonlinear systems from any selected desired output space; ii) the calculated DIS* satisfies the specifications and constraints associated with design conditions; and iii) considering that the user could identify the necessary modifications to the reactor design to reach specific targets, this new framework could be employed as a strategy for making dramatic reductions in the size of a chemical process so as to reach a given production objective. Following these conclusions, the next chapter shows the extension of problem P1 to incorporate process intensification into process design of nonlinear systems. 


\section{Chapter 5 Incorporation of Process Intensification into Operability Approach}

Figure 5.1 shows a schematic of the process operability calculations carried out thus far (boxes with solid lines), as well as the steps where the incorporation of process intensification could be performed (boxes with dashed lines). The analysis of such incorporation of intensification targets, such as footprint reduction and efficiency increase, into the operability calculations is described in this chapter.

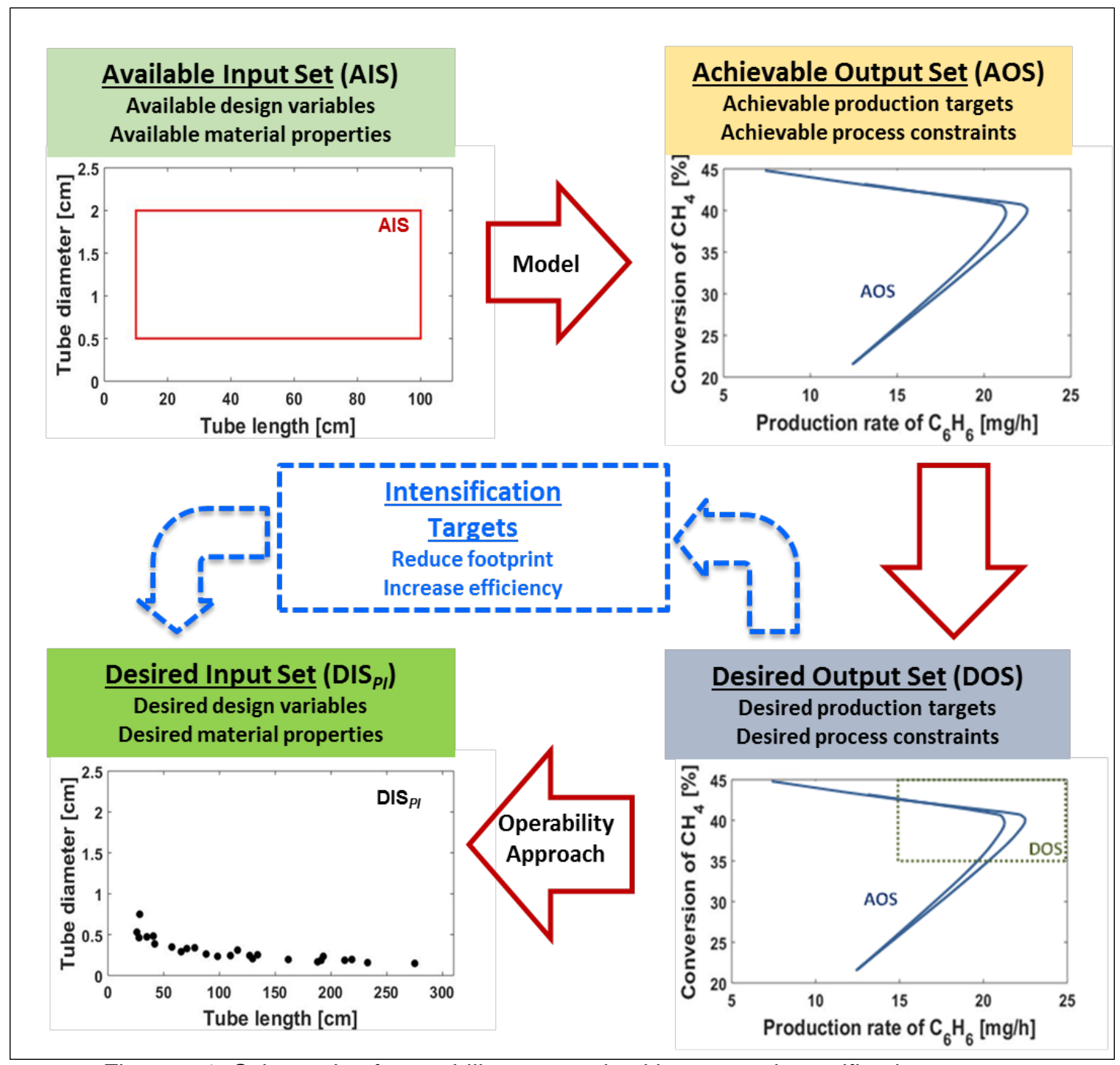

Figure 5.1. Schematic of operability approach with process intensification 


\subsection{Problem Formulation}

A new objective function $(\Omega)$ is formulated to minimize the selected process intensification target, $\mathrm{Pl}_{\text {target, }}$ for instance process footprint (e.g., volume, area), subject to a desired level of performance. This new objective function can be expressed as

$$
\begin{aligned}
\text { P2: } \Omega=\min _{\mathrm{u}_{P I}^{*}}\left[\mathrm{PI}_{\text {target }}\right] \\
\text { subject to: } \mathrm{u}_{P I}^{*} \in \text { DIS }^{*} \\
\mathbf{y}_{P I} \in \text { DOS }^{*}
\end{aligned}
$$

in which the obtained optimal point in terms of intensification for the input variable can be represented by $\mathrm{u}_{P I}^{*}$. The desired level of performance (e.g., conversion and production rate) corresponds to a subset of the $\mathrm{DOS}^{*}$ and can be defined as follows:

$$
\mathrm{DOS}_{P I}=\left\{\mathbf{y}_{P I} \mid \mathbf{y}_{P I}=\left(\mathrm{y}_{P I_{1}}, \mathrm{y}_{P I_{2}} \ldots \mathrm{y}_{P I_{l}} \ldots \mathrm{y}_{P I_{q}}\right)\right\}
$$

in which " $q$ " represents the points in $\operatorname{DOS}_{P I}, q \leq p$.

The desired input set for process intensification $\left(\mathrm{DIS}_{P I}\right)$ is then obtained by implementing the optimization-based algorithm, $\mathrm{P} 1$, for the $\mathrm{DOS}_{P I}$ points:

$$
\mathrm{DIS}_{P I}=\left\{\mathbf{u}_{P I} \mid \mathbf{u}_{P I}=\left(\mathrm{u}_{P I_{1}}, \mathrm{u}_{P I_{2}} \ldots \mathrm{u}_{P I_{l}} \ldots \mathrm{u}_{P I_{q}}\right)\right\}
$$

Note that the DIS $P I$ will also be a subset of the DIS*. The subscript $l=1,2,3 \ldots q$ represents the point index inside the process intensification space. The application of problem P2 is described in the examples below. 


\subsection{DMA-MR Case Studies Revisited}

\subsubsection{DMA-MR case study $(2 \times 2)$}

The process intensification target considered here is to reduce the DMA-MR footprint characterized by the minimization of the reactor volume and membrane surface area. The $2 \times 2$ example in Chapter 4 is first employed for the incorporation of process intensification targets into the operability approach (see Figure 4.4). Initially, 100 points are selected to represent the input, DIS*, and output, DOS*, spaces. These points are shown in Figure 5.2 (see black dots). For this example, the desired level of performance for the outputs is specified as the production rate of benzene greater than 20 [mg/h] (depicted in Figure 5.2 as hollow red circles), corresponding to the $\mathrm{DOS}_{P /}$ of $q=50$ points.
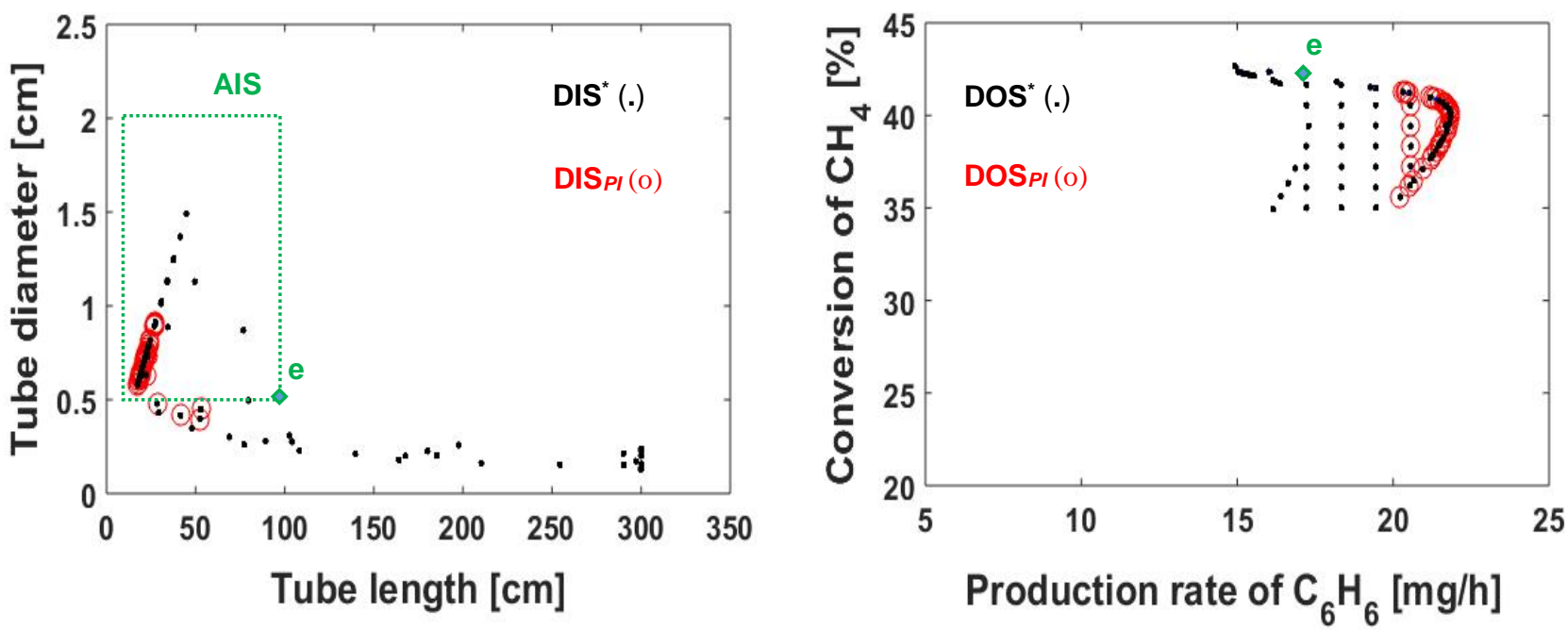

Figure 5.2. 2-D DMA-MR system. AIS, DIS* and DIS ${ }_{P I}$ (on the left).

$\mathrm{DOS}^{*}$ and $\mathrm{DOS}_{P I}$ (on the right) 
P2 is then solved for these selected points considering the intensification target of footprint reduction and the intensified reactor design obtained presents a length of 17.38 [cm] and a diameter of $0.58[\mathrm{~cm}]$. Comparing the intensified with the base case design (see Figure 5.2, point "e"), the DMA-MR design shows footprint reduction by approximately $77 \%$ in volume of reactor and $80 \%$ in membrane surface area for an equivalent level of performance. This case study indicates that the developed operability tool may enable the incorporation of process intensification targets during early stages of process design for membrane reactors and other complex chemical processes.

A schematic framework for the optimization-based approach as a tool for process intensification is outlined in Figure 5.3. In particular, this figure summarizes all the calculation steps detailed above considering process targets and constraints. These steps include the traditional operability path (see dotted black lines) and the newly formulated optimization approach for the computation of DIS* (dashed red lines) and for process intensification (solid blue lines). 


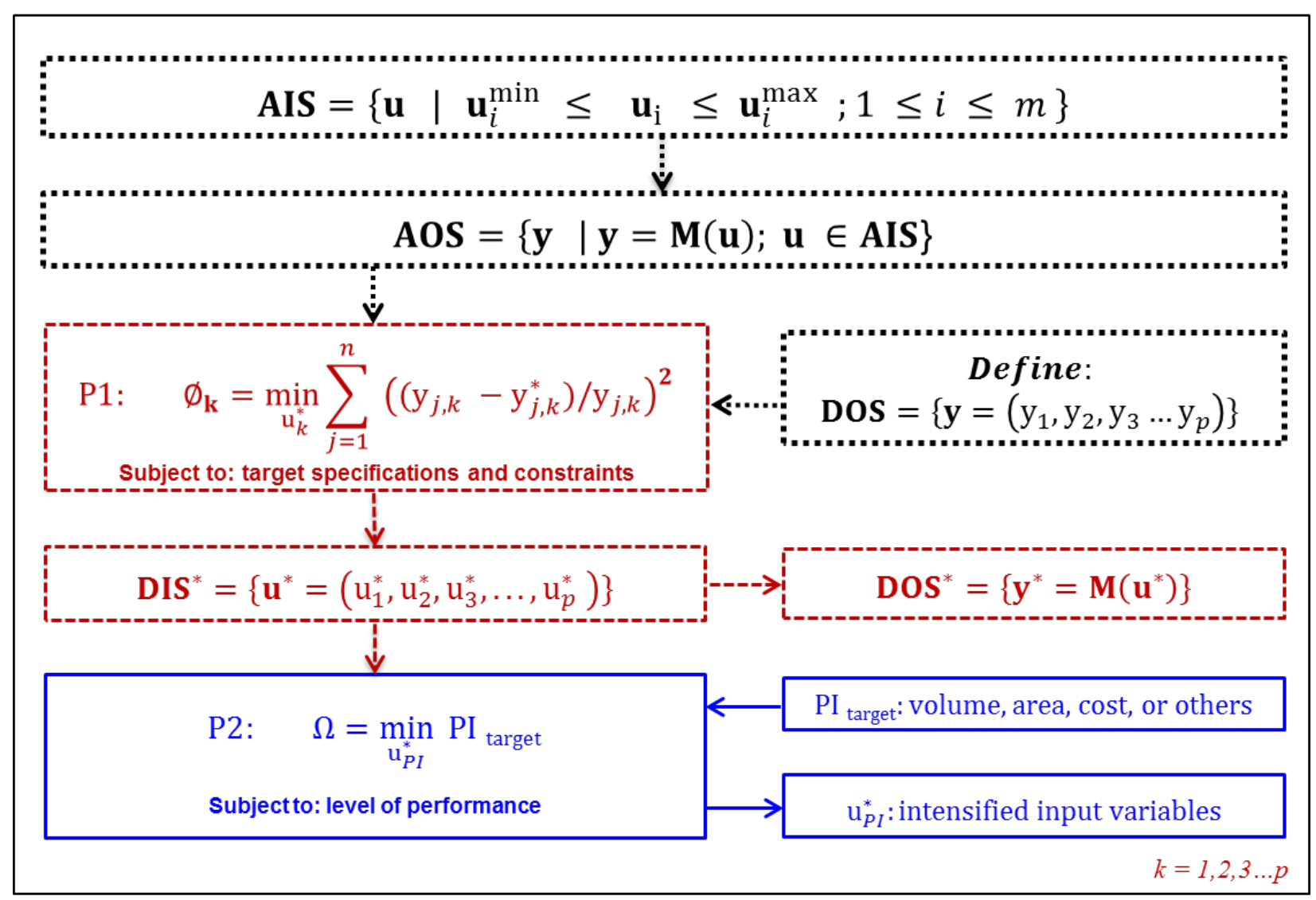

Figure 5.3. Mathematical approach of process operability for intensification

\subsubsection{DMA-MR case study (3x3)}

Now revisiting the DMA-MR 3×3 case study presented in Chapter 4 , the calculated feasible DOS points, or DOS*, for this system are shown in Figure 5.4 (see solid blue dots on the right, with some points superposed due to figure orientation). These points are obtained directly as a result of the optimization problem P1 by storing the feasible output points for each optimal input point. The DOS* is composed of 125 points spread around reduced ranges when compared to the original DOS in Figure 4.5. These ranges are characterized by benzene and hydrogen production rates, and methane conversion of 
approximately $15.91-24.84[\mathrm{mg} / \mathrm{h}], 1.76-2.06[\mathrm{mg} / \mathrm{h}]$, and $36.05-42.48[\%]$, respectively.
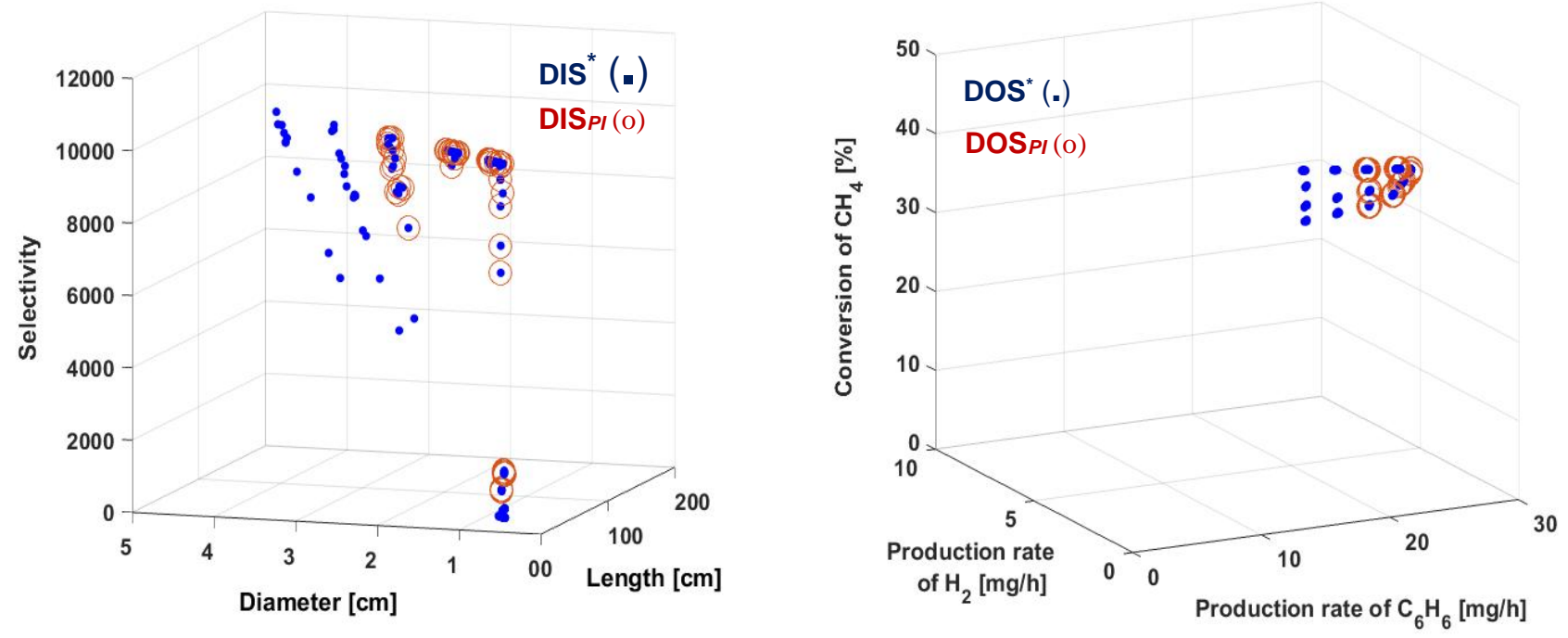

Figure 5.4. 3-D DMA-MR system. Operability sets: DIS* and DIS ${ }_{P I}$ (on the left).

$\mathrm{DOS}^{*}$ and $\mathrm{DOS}_{P I}$ (on the right)

The DOS* can now be further analyzed using the optimizer P2 to enable the reduction of the DMA-MR footprint considering the intensification targets of minimizing the reactor volume and membrane surface area. For this problem, the 125 points of DIS* from P1 are considered as input constraints and the desired level of performance for the outputs is specified as the production rate of benzene greater than $20[\mathrm{mg} / \mathrm{h}]$. Among the initially analyzed points, 75 that satisfy the level of performance constraint are obtained to define the DIS $S_{P I}$ and $D_{D O S}$ as depicted in Figure 5.4 (see hollow red circles). Among these points, the intensified reactor design with smallest footprint consists of a length of 17.89 [cm], a diameter of $0.59[\mathrm{~cm}]$, and membrane selectivity of 1520 . The intensified output point corresponds to benzene and hydrogen production rates, and a methane conversion of $24.84[\mathrm{mg} / \mathrm{h}], 2.06[\mathrm{mg} / \mathrm{h}]$, and 42.48 [\%], respectively. This intensified 
design presents footprint reduction of approximately 75 [\%] in volume of reactor and 79 [\%] in membrane surface area when compared to a base case design (e.g., for the calculated catalytic MR volume of $19.6\left[\mathrm{~cm}^{3}\right]$ in Chapter 3).

As the first step in the extension of the operability analysis from first-principles models to process simulator runs, the following case study introduces a large-scale NGCC power plant model built in Aspen Plus ${ }$.

\subsection{NGCC Power Plant Case Study}

\subsubsection{Process description}

The large-scale NGCC power plant model developed in Aspen Plus considers the following main process units: air compressor, combustion chamber with dry low $\mathrm{NO}_{\mathrm{x}}$ burner, gas turbine, heat recovery steam generator (HRSG), steam turbine, and condenser. Figure 5.5 depicts a simplified schematic of the NGCC power plant considered here. The communication between the operability tools developed in MATLAB and the model in Aspen Plus is carried out using a Visual Basic for Applications (VBA) in Excel interface. In particular, the operability algorithms programmed in MATLAB call through the interface the process model in Aspen Plus for obtaining the relationship between the input and output spaces for different simulator runs. Available data from national laboratories (DOE/NETL, 2013; DOE/NETL, 2015) and industrial processes, 
including micro turbines (Capstone, 2016), are used to help defining the constraints for the design of the NGCC system.

Specifically, the process model for this example is built based on the information presented in the U.S. Department of Energy (DOE) report (DOE/NETL, 2013), in which the main pieces of equipment considered are two GE 7FA.05 gas turbines, two triple pressure level single reheat type HRSGs, and one condensing steam turbine. The ambient air and natural gas (assuming $52,314 \mathrm{~kJ} / \mathrm{kg}$ as the higher heating value) are combined in a dry low $\mathrm{NO}_{x}$ burner with a temperature of $1632[\mathrm{~K}]$, the flue gas exits the gas turbine at $877[\mathrm{~K}]$ and proceeds to the HRSG. The produced steam in the HRSG at a temperature of approx. $840[\mathrm{~K}]$ serves as the inlet stream for the steam turbine. The flue gas exits the HRSG at $361[\mathrm{~K}]$ and proceeds to the stack. The condenser is cooled using water from the cooling tower (see DOE report for other operating conditions).

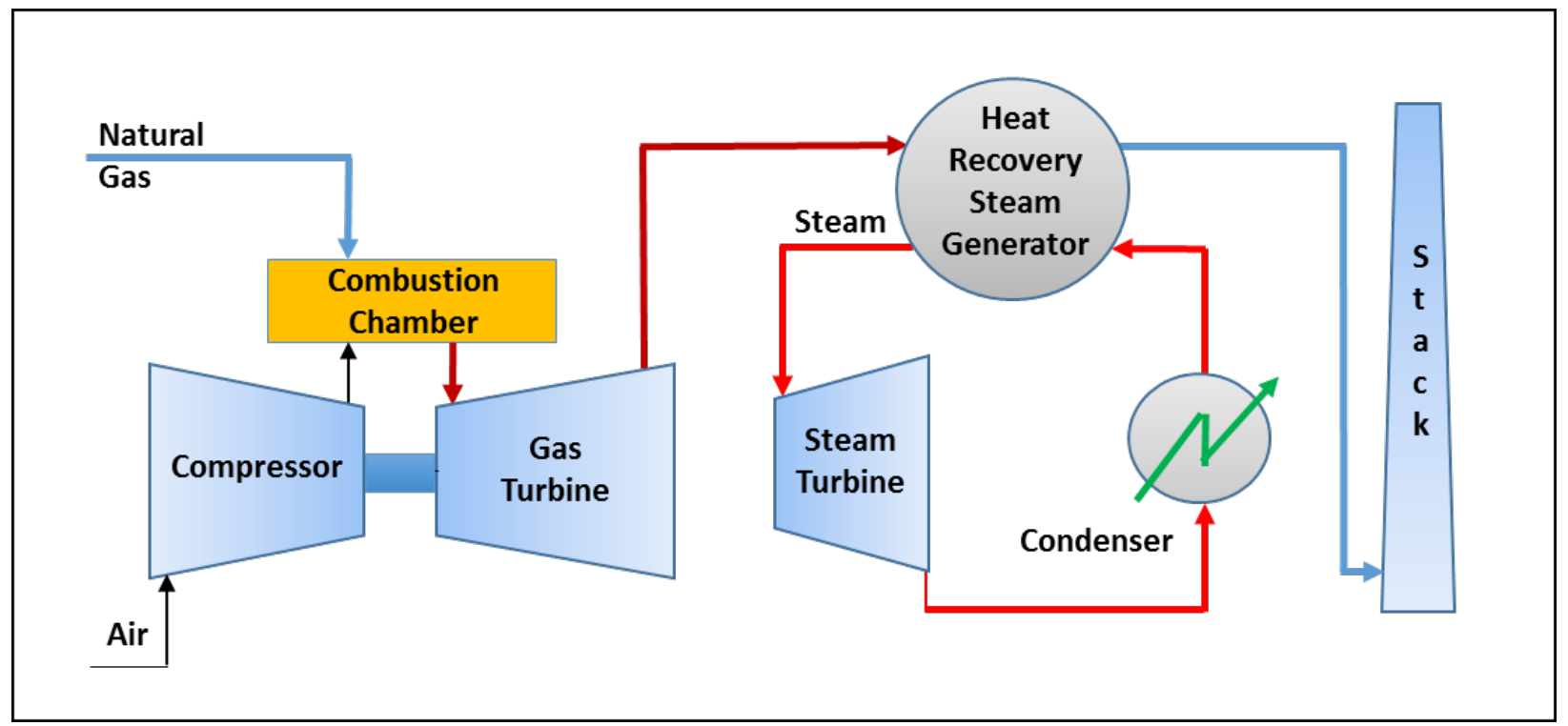

Figure 5.5. Simplified schematic of NGCC power plant 


\subsubsection{Operability analysis}

The AIS considered here is represented by a $2 \times 2$ system, in which the input variables correspond to the natural gas inlet flow and the generated steam flow from the HRSG that feeds the steam cycle. The selected inputs are also considered as attributes for scale up as the design of the NGCC gas and steam turbine cycles can be enlarged or downsized according to their inlet streams. For instance, as the natural gas flow is reduced, the size of the gas turbine portion of the plant is downsized. Similar behavior occurs to the steam flow with respect to the steam turbine cycle. The net plant power and the net plant efficiency are the outputs that specify the AOS. The net plant power is defined in this study as the combined gas turbine and steam turbine power generation with the deduction of the air compressor power usage. The net plant efficiency is given by the ratio between the net plant power and the total energy content in the natural gas flow (in higher heating value basis). The input ranges considered for the operability analysis for the natural gas and steam flows are $20-100$ [ton/h] and $100-1000$ [ton/h], respectively. These input ranges are chosen to allow the calculation of output ranges by process simulator runs for the net plant power and net plant efficiency between $100-800$ [MW] and $36-55$ [\%], respectively. Both input and output ranges are depicted in Figure 5.6. The obtained output ranges using the Aspen model were validated against the available information from the literature cited above. In particular, the calculated power range is consistent with the conventional range for NGCC systems. 

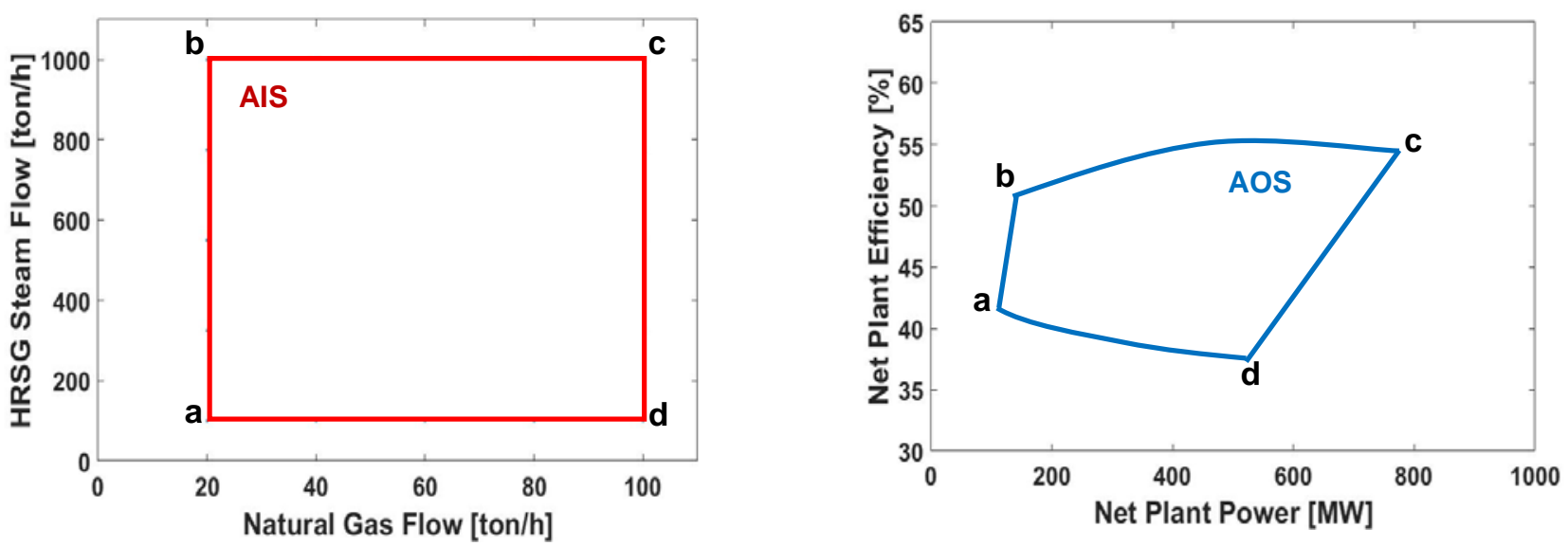

Figure 5.6. NGCC power plant. Natural gas and HRSG steam flows as input variables (AIS on the left). Net plant power and efficiency as process outputs (AOS on the right)

For performing the operability analysis, a grid was built by assuming that the input ranges were equally divided by 5 , which corresponded to increments of 20 [ton/h] and 225 [ton/h], for the gas and steam flows, respectively. Figure 5.6 presents the defined AIS and the calculated AOS for this example. The operability calculations shown in this figure suggest that the higher net plan efficiencies are obtained for the highest steam flow rate value from the HRSG, represented by the segment "b" to "c", as expected due to the greater presence of the steam cycle. In contrast, the segment from points "a" to "d" presents lower net plant efficiencies, as the steam flow to the turbine is the lowest. The efficiency values in this segment are consistent with the low efficiencies of existent modular gas turbines for electricity generation. Note that in practice, some of these regions may be infeasible. However, the operability mapping still provides insight on the relationship between inputs and outputs. Regarding the effect of the natural gas flow on the output variables, it is worth pointing out the significant differences in efficiencies for the highest natural gas flow value of $100[$ ton/h] in the segment " $c$ " to " $d$ ". Thus, the 
performed operability analysis shows that there is an opportunity to systematically find different combinations of gas and steam turbine cycle conditions that can potentially enable intensified and even modular NGCC designs.

\subsubsection{Process design and intensification}

To start analyzing process conditions of NGCC plants towards intensified designs, the desired output requirements (power, efficiency) can be included in the operability analysis as the DOS (see Figure 5.7 on the right, dashed green rectangle). For such analysis, the natural gas flow $\left(u_{1}\right)$ and the HRSG steam flow $\left(u_{2}\right)$ define the input space as above, and the net plant power $\left(\mathrm{y}_{1}\right)$ and net plant efficiency $\left(\mathrm{y}_{2}\right)$ specify the DOS.
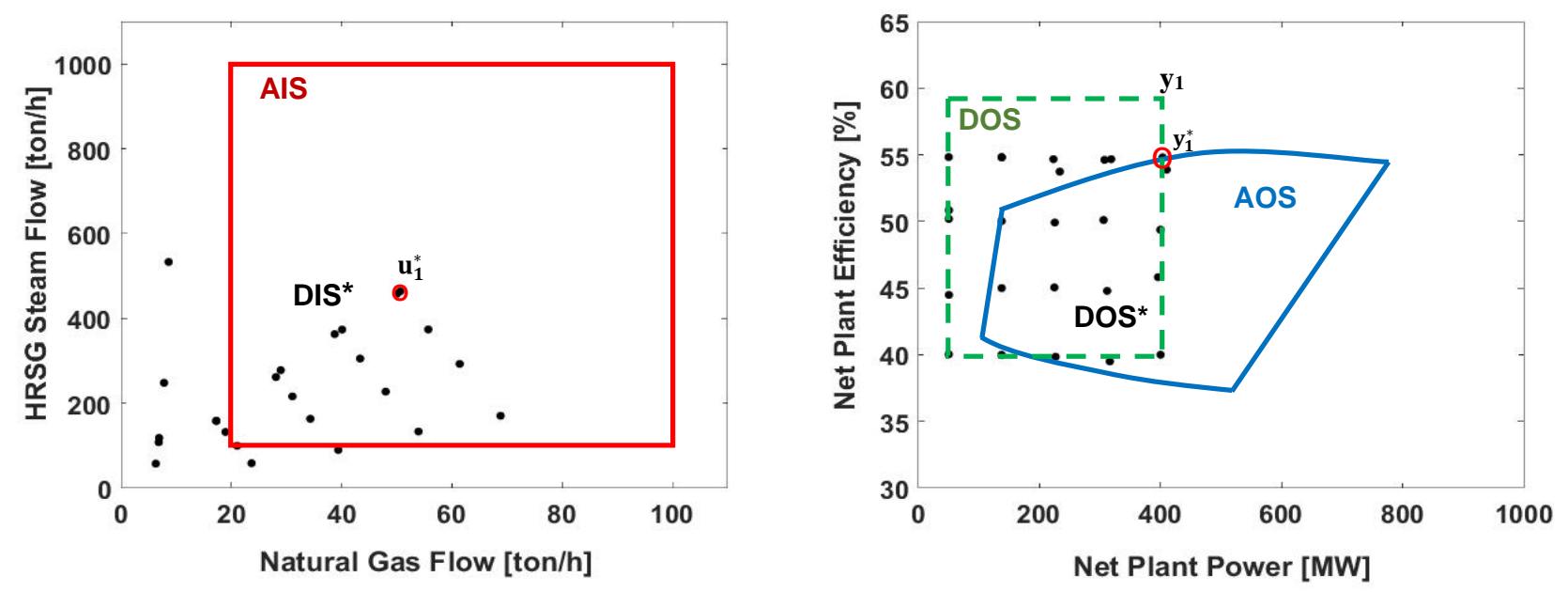

Figure 5.7. NGCC power plant. Operability sets: AIS and DIS* (on the left).

AOS, DOS and DOS* (on the right)

For the operability computations, the desired ranges selected for $\mathrm{y}_{1, k}$ and $\mathrm{y}_{2, k}$ are between $50-400[\mathrm{MW}]$ and $40-60[\%]$, respectively, which are discretized considering 
$p=25$ points $(5 \times 5$ grid), thus $k=1,2,3 \ldots 25$. These ranges represent desired operating regions for intensified/modular NGCC plants with reduced size and maximized efficiency that have not been widely explored in the literature. Using these DOS specifications, the optimization-based approach defined in P1 is employed for the calculation of the DIS* as presented in Figure 5.7 (on the left). For the DIS* calculation, positivity constraints on both $\mathrm{u}_{1, k}$ and $\mathrm{u}_{2, k}$ are imposed. As an example of the evaluation of $\mathrm{P} 1$, for the first point, $k=1$, in the DOS (see Figure 5.7, point $\left.\mathrm{y}_{1}\right), \mathrm{y}_{1}=\left(\mathrm{y}_{1,1}, \mathrm{y}_{2,1}\right)=(400,60)$, and considering an initial guess for the inputs, $u_{1, O}^{*}=(20,100)$, the calculated feasible input point is $u_{1}^{*}=$ $\left(u_{1,1}^{*}, u_{2,1}^{*}\right)=(50,464)$. Also, by the optimizer P1, the feasible output point $\left(y_{1}^{*}\right)$ related to the calculated $u_{1}^{*}$ is obtained as $y_{1}^{*}=\left(y_{1,1}^{*}, y_{2,1}^{*}\right)=(400,55)$ (see hollow red circles in Figure 5.7). Following a similar procedure for all points, the optimizer P1 can calculate all of the feasible input points inside the DIS* from the DOS established points in the grid. The calculated DIS* shown in Figure 5.7 as solid black dots (on the left) characterize an input region with approximated ranges for the natural gas and steam flows between 6 76 [ton/h] and $58-533$ [ton/h], respectively. For the outputs, the operability calculations indicate that the highest net plant efficiency achievable for the NGCC is around 55\%, considering the employed data and the studied range for the net power plant. This efficiency value is consistent with the previously reported values (DOE/NETL, 2013). This percentage thus does not satisfy the $60 \%$ efficiency that was established as desired output due to thermodynamic limitations associated with the integration of gas and steam turbine cycles. 
The reduction of the net plant power from 400 to 50 [MW] is analyzed next as an effort to demonstrate the application of P2 for process intensification. In particular, the level of performance and process intensification target are defined as the maximization of the net plant efficiency and reduction of the NGCC footprint characterized by the net plant power, respectively. In this case, the desired level of performance for the output corresponds to efficiency values greater than or equal to $52.5 \%$. Problem P2 is then solved considering the 25 points from the DIS* and DOS*, and the solution points that satisfy the performance level are shown in Figure 5.8 (see hollow red circles). It is worth noting that Figure 5.8 (on the left) suggests a linear relationship between the input variables, natural gas and steam flows. This relationship can be expressed as $\mathrm{u}_{2, k}=9.15$ $\mathrm{u}_{1, k}$ and occurs when the desired net plant efficiency is around 55 [\%]. This relationship can be explained by the maximum heat exchanged amount in the HRSG that is achieved when the ratio between natural gas and steam flows is approx. 9.15.
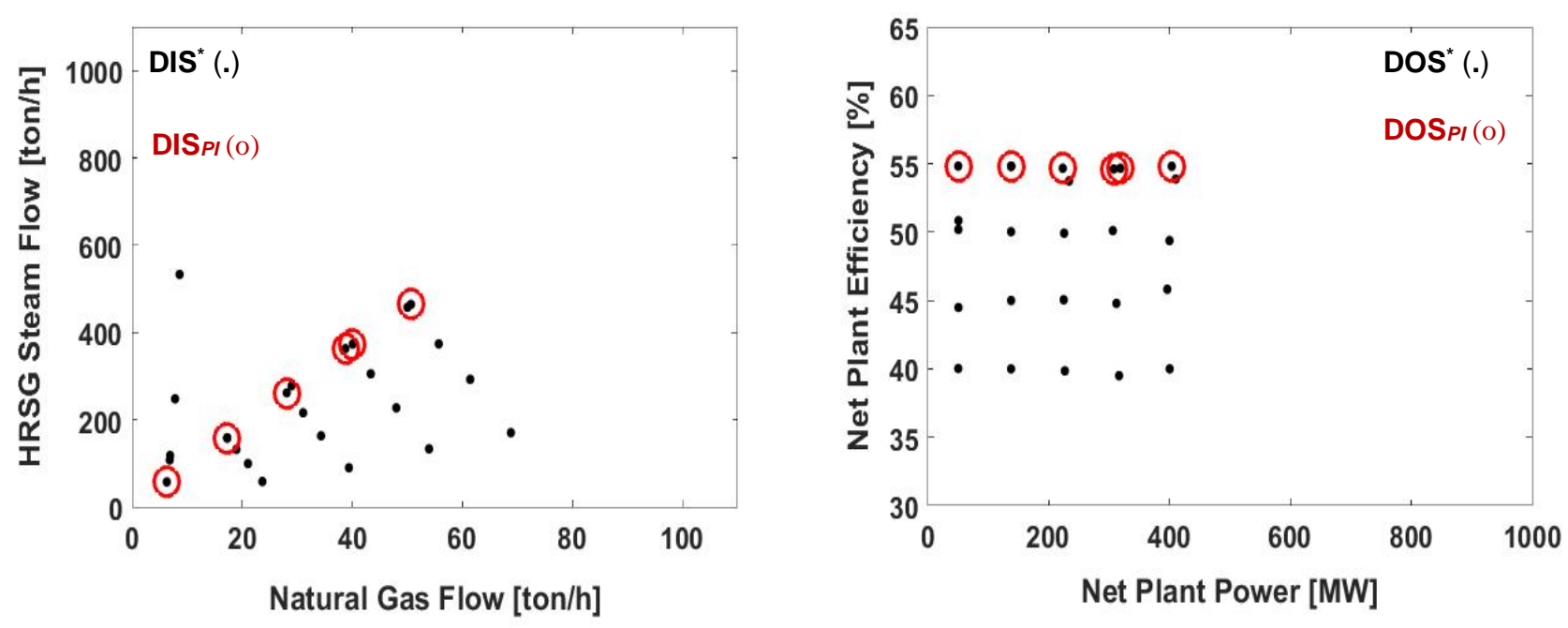

Figure 5.8. NGCC power plant. Operability sets: $D_{I}{ }^{\star}$ and DIS $S_{P I}$ (on the left). $\mathrm{DOS}^{*}$ and $\mathrm{DOS}_{P I}$ (on the right) 
The net plant efficiency is reduced when the steam flow does not satisfy the calculated optimal ratio. For example, when the steam flow is low, the steam turbine generates proportionally less amount of electricity. On the other hand, when the generated steam flow is greater than the ratio, its temperature is lower than $840[\mathrm{~K}]$ and thus, the net enthalpy flow decreases causing a reduction in the steam turbine power. Finally, the most intensified/modular NGCC system produced by P2 presents a net plant power of 50 [MW], a net plant efficiency of 54.8 [\%], a natural gas flow of 6.2 [ton/h], and a HRSG steam flow of 57.6 [ton/h]. This example thus shows the specifications that could be achieved to enable an NGCC system of 50 [MW], considering the developed Aspen model of the system.

The two analyzed case studies in this chapter demonstrated that the formulated optimization problems P1 (to calculate the feasible DIS from the DOS) and P2 (to minimize the selected process intensification target) may be employed as tools for design and intensification of chemical and energy systems. They also suggest that P1 could be incorporated into P2 resulting in a nested bilevel optimization problem, providing a more systematic and streamlined optimization formulation (Sinha et al., 2014). In that case, the full DOS with " $p$ " points would be considered and the outcome would be the same as if P1 and P2 were solved in sequence. The integrated framework based on bilevel optimization, in which each problem can be formulated in separate levels, is presented in the next chapter. 


\section{Chapter 6 Bilevel Operability Framework}

A hierarchical structure for combining P1-P2 in one step is presented in this chapter in which the optimal solutions of the inner optimization task (argmin), or lower level, are accepted as input variables for the outer optimization task, or upper level (Sinha et al., 2014). Two case studies are revisited to validate the intensified input and output outcomes from the new operability framework developed in this chapter. Also, the computational times for the AOS and DIS* calculations for different DMA-MR subsystems are analyzed.

\subsection{Problem Formulation}

For the bilevel operability framework, the inner optimization task (P1) finds the desired input points by minimizing the relative error between the desired and feasible output points, subject to constraints and target specifications. Then, the outer optimization task (P2) provides the intensified design by minimizing the process intensification objective function, by taking all or part of the found desired input points in the lower level (depending on the selected level of performance in the DOS). The proposed bilevel optimization problem can be mathematically structured as follows: 


$$
\begin{array}{ll}
\text { P3: } & \Psi=\underset{\mathrm{u} \in \mathbb{U}}{\min }\left[\mathrm{PI}_{\text {target }}\right] \\
\text { subject to: } & \mathrm{u}_{k}^{*} \in \underset{\substack{\mathrm{u}_{k}^{*} \in \mathbb{U}_{k} \\
\mathbf{y}_{k} \in \mathrm{DOS}}}{\operatorname{argmin}}\left\{\sum_{j=1}^{n}\left(\frac{\mathrm{y}_{j, k}-\mathrm{y}_{j, k}^{*}}{\mathrm{y}_{j, k}}\right)^{2}\right\} \\
& \mathbf{c}_{1}\left(\mathrm{u}_{k}^{*}\right) \leq \mathbf{0}
\end{array}
$$

in which $\mathbb{U} \in \mathbb{R}_{\geq 0}^{m}$ and $\mathbb{U}_{k} \subset \mathbb{U}$. Also, the variables are defined as in the descriptions of $\mathrm{P} 1$, P2 above. Additionally, in this formulation, the DOS in a discretized grid, the level of performance and the target specifications can be incorporated at once as process constraints, and an initial input guess that is within the AIS is required to start the optimizer evaluations. It is worth mentioning that prior AOS calculations can provide an insight to the user on how to select the DOS. For instance, if the user would like to push a certain performance variable value towards a more desired value, this factor could be included in the specification of the corresponding DOS dimension.

The solution for this problem considering $k=1,2,3 \ldots p$ points in a grid provides the intensified input and output values $\left(\mathrm{u}_{P I}^{*}, y_{P I}^{*}\right)$. The calculated outcome thus provides conditions for enabling the design of individual intensified/modular units considering desired output specifications and intensification targets. To solve this optimization problem, NLP subroutines available in the Optimization toolbox in MATLAB ("fmincon", "fminsearch", or equivalent) can be used. For the performed case studies in this thesis, "fminsearch" in MATLAB was successfully employed. 


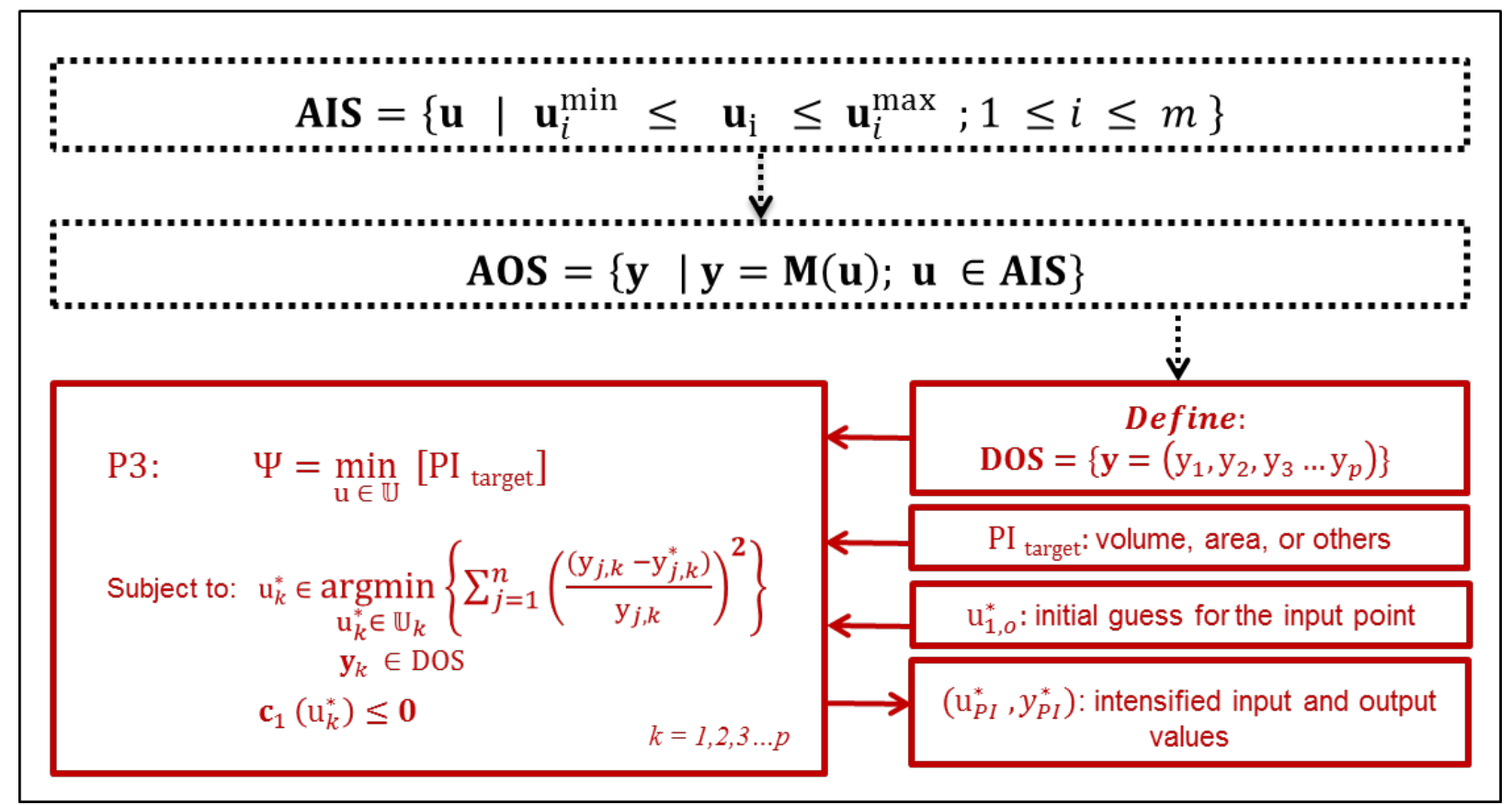

Figure 6.1. Schematic diagram of the bilevel optimization-based operability framework for process intensification

A schematic framework for this bilevel optimization-based operability approach as a tool for process intensification is outlined in Figure 6.1. In particular, this figure summarizes all the calculation steps detailed above considering process targets and constraints. These steps include the traditional operability path (see dotted black lines) and the newly formulated bilevel optimization approach for the computation of the intensified input and output points (solid red lines). For illustration of this novel bilevel optimization-based operability framework, the two case studies presented above are revisited. The first corresponds to the DMA-MR system (as $2 \times 2$ and $3 \times 3$ examples) described by a first-principles model, and the second is the NGCC power plant model built in Aspen Plus. 


\subsection{Case Studies Revisited}

\subsubsection{DMA-MR}

Before revisiting the $3 \times 3$ example presented in subsection 5.2.2, a 2×2 DMA-MR system with similar conditions as in subsection 5.2.1 is revisited as a lower-dimensional subsystem of the MR example. For this problem, the following conditions are specified to solve the bilevel optimization problem, P3: (i) process intensification targets are the reduction of reactor volume and membrane surface area; (ii) level of performance is set as the production rate of benzene greater than 20 [mg/h]; (iii) DOS is discretized as a grid of 100 points for the selected benzene production rate and methane conversion outputs in the ranges between $15-25[\mathrm{mg} / \mathrm{h}]$, and $35-45$ [\%], respectively (the domains for the benzene production rate and methane conversion are divided by 10, which correspond to increments of 1 [mg/h] and 1 [\%], for each variable, respectively); (iv) positivity constraints are imposed on the length and diameter input variables; and (v) design constraints, such as length over diameter greater than 30 and the reactor length less than $300[\mathrm{~cm}]$, are assumed.

For solving this problem, the initial input guess to start the optimizer evaluations is set as (length, diameter $)=(10,0.5)$. Figure 6.2 depicts the outcome obtained for the intensified modular reactor design by solving P3. Note that the intensified reactor length and diameter are $17.38[\mathrm{~cm}]$ and $0.58[\mathrm{~cm}]$, respectively (see Figure 6.2, solid red dot on the left). This result is the same as the one obtained by solving P1 and P2 in series (see 
subsection 5.2.1), thus validating the proposed bilevel approach. The output point associated with the intensified input variables corresponds to 20.2 [mg of benzene/h] and 35.5 [\%] of methane conversion (see Figure 6.2, solid red dot on the right).
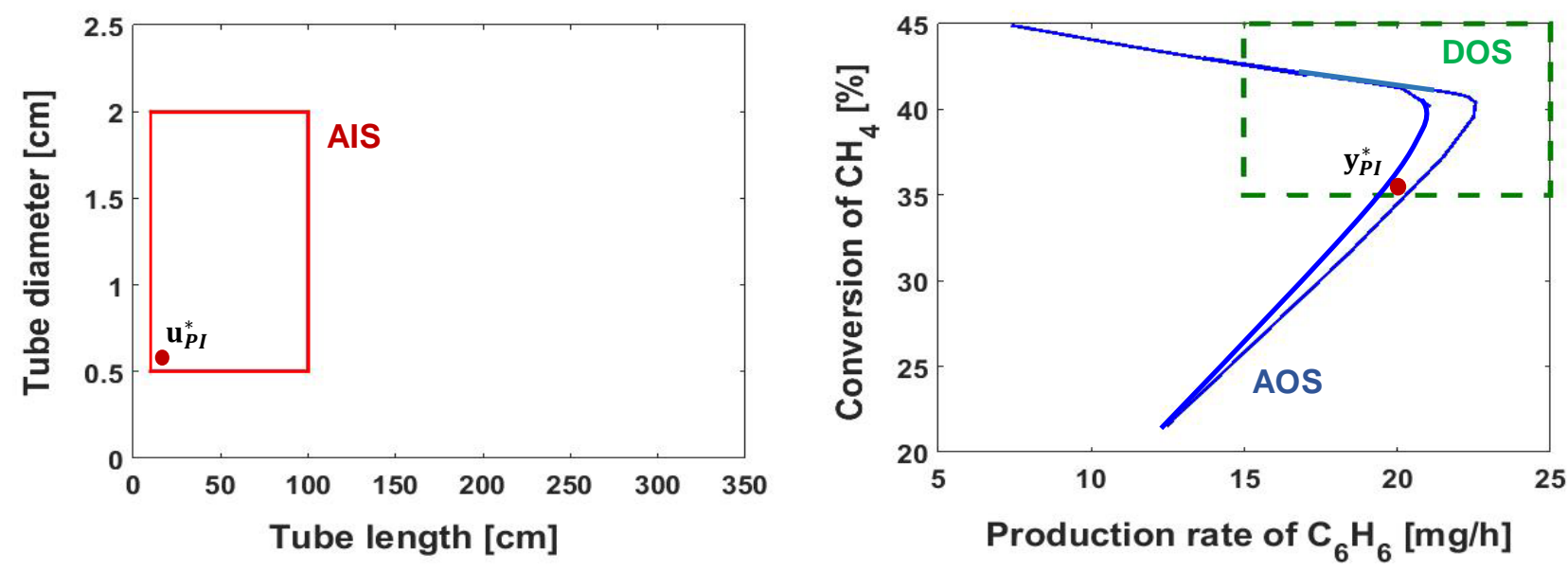

Figure 6.2. 2-D DMA-MR system. Intensified input point and AIS (on the left). Intensified output point, AOS and DOS (on the right)

After the conclusion of the $2 \times 2$ DMA-MR subsystem, the $3 \times 3$ example presented above is revisited using the new integrated framework. Specifically, the bilevel optimization problem is employed here to calculate the intensified DMA-MR design in one step. Problem P3 is solved for the following conditions detailed above and summarized here for clarity: the DOS is defined for the selected benzene and hydrogen production rates, as well as methane conversion in the ranges, $15-25[\mathrm{mg} / \mathrm{h}], 3-6[\mathrm{mg} / \mathrm{h}]$, and 35 45 [\%], respectively. These ranges are discretized considering 125 points (i.e., the domains for the benzene and hydrogen production rates, and methane conversion are divided by 5 , which correspond to increments of, $2.5[\mathrm{mg} / \mathrm{h}], 0.75[\mathrm{mg} / \mathrm{h}]$, and $2.5[\%]$, for each variable, respectively; thus $k=1,2,3 \ldots 125)$. Positivity constraints on the length and 
diameter are considered. Also, design specifications are set, such as membrane selectivity less than 10,000 (closer to expected property that could be obtained in the lab), reactor length over diameter greater than 30 , and length less than $300[\mathrm{~cm}]$. Finally, the initial input point guess (length, diameter, and selectivity) to start the optimizer evaluations is $(20,0.5,1000)$, the process intensification targets to minimize are the catalytic reactor volume and membrane surface area, and the level of performance is specified as the production rate of benzene greater than $20[\mathrm{mg} / \mathrm{h}]$.
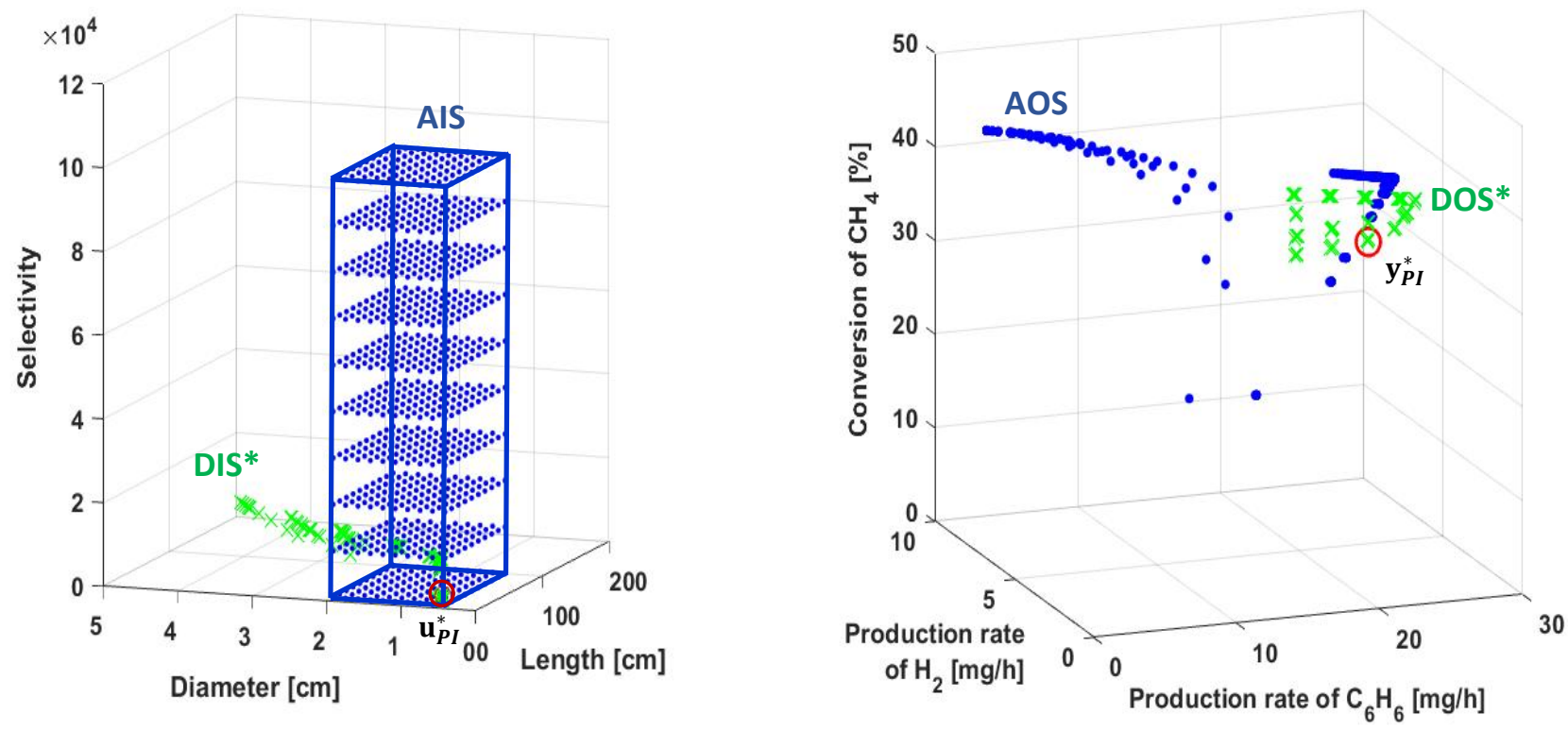

Figure 6.3. 3-D DMA-MR system. Operability sets: AIS, DIS* and $\mathrm{u}_{P I}^{*}$ (on the left). $\mathrm{AOS}, \mathrm{DOS}^{*}$ and $\mathrm{y}_{P I}^{*}$ (on the right)

The calculated results by P3 are showed in Figure 6.3. These results indicate that the intensified catalytic membrane reactor presents a length and diameter of $17.89[\mathrm{~cm}]$ and 0.59 [cm], respectively, and a membrane selectivity of 1520 (see Figure 6.3, hollow red circle on the left). Also, the intensified output point corresponds to 24.84 [mg of 
benzene/h], 2.06 [mg of hydrogen/h], and 42.48 [\%] of methane conversion (see Figure 6.3, hollow red circle on the right). In Figure 6.3, the AIS (blue parallelepiped on the left) and the AOS (solid blue circles on the right) from the original operability analysis are also shown for comparison. The points in the DIS* and DOS* are also represented with " $x$ " in the figure to show that the intensified point is contained in these sets according to the P3 definition. Thus, the calculated intensified input and output values are consistent with the values obtained by running $\mathrm{P} 1$ and $\mathrm{P} 2$ in sequence (see subsection 5.2.2). This result demonstrates that P3 can be employed as a one-step bilevel-type optimization tool for process design and intensification. In particular, the intensified MR design presented a footprint reduction of approx. 75 [\%] in volume of reactor and 79 [\%] in membrane surface area (when compared to the base case). These results indicate that the DMA-MR has potential to be modularized in the future.

\subsubsection{NGCC power plant}

The NGCC plant using the information described in subsection 5.3 is revisited in this subsection by employing the new bilevel optimization formulation. In particular for P3, the outer level objective function corresponds to the minimization of the net plant power, as the process intensification target, subject to the following set of conditions summarized here: (i) level of performance is set as a net plant efficiency greater than 52.5 [\%]; (ii) DOS is defined by the net plant power and efficiency with ranges between $50-400$ [MW] and $40-60[\%]$, respectively. This set is once again discretized considering $p=25$ points (5x5 grid), thus $k=1,2,3 \ldots 25$; (iii) positivity constraints are imposed on the selected input 
variables, natural gas and steam flows. To start the optimization, the initial input point with coordinates $(20,100)$ is chosen.
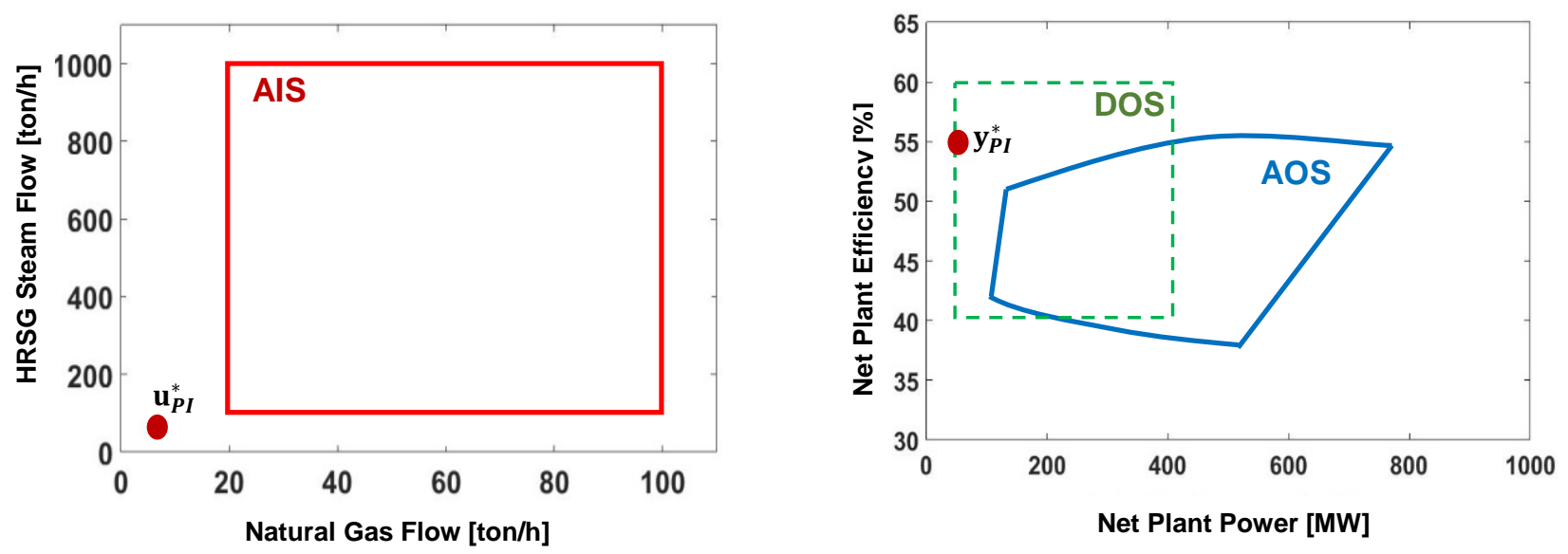

Figure 6.4. NGCC power plant. Intensified input point and AIS (on the left). Intensified output point, AOS and DOS (on the right)

The obtained results from the optimizer are consistent with the example in subsection 5.3.3 associated with the process intensification of the NGCC power plant. In particular, the natural gas flow of 6.2 [ton/h] and HRSG steam flow of $57.6[$ ton $/ \mathrm{h}]$ are calculated as the point for the intensified NGCC modular plant. This input point maps into output coordinates of a net plant power of $50[\mathrm{MW}]$ and a net plant efficiency of 54.8 [\%] (see solid red dots in Figure 6.4).

A new case is now considered for the application of $\mathrm{P} 3$ towards enabling the design of even smaller modular NGCC power plants. Figure 6.5 depicts the AIS for a modular system with ranges between $0.08-0.40[$ ton/h] and $0.40-4.00[$ ton/h] for the natural gas and steam flows, respectively. These selected input ranges allow the calculation of 
modular steam and gas turbines for electricity generation with individual cycle (gas, steam) efficiencies approx. between 25 - 40 [\%]. For these inputs, the calculated ranges for the AOS represented as the net plant power and efficiency are approx. $0.5-3.2[\mathrm{MW}]$ and $37-55[\%]$, respectively.
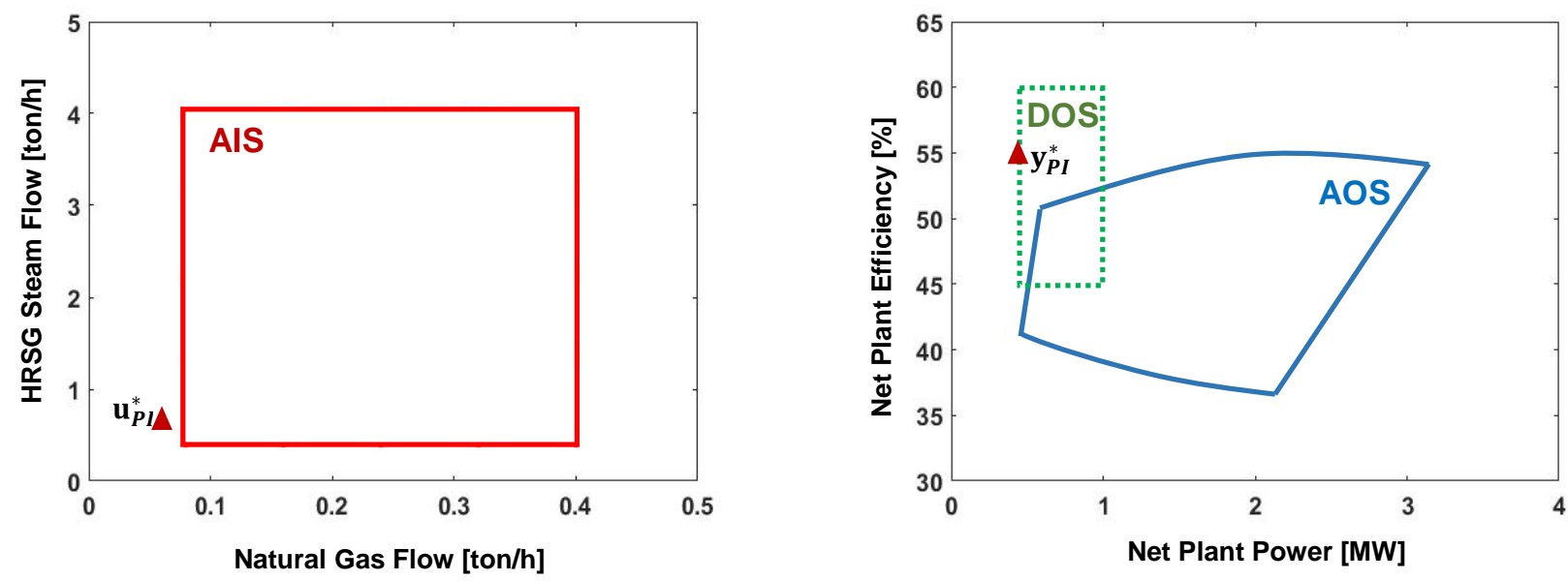

Figure 6.5. Towards modular NGCC plant: intensified input point and AIS (on the left). Intensified output point, AOS and DOS (on the right)

The specified DOS is also depicted in Figure 6.5 (see dotted green rectangle) with ranges between $0.5-1.0[\mathrm{MW}]$ and $45-60$ [\%], respectively. Problem P3 employs once again as the outer objective function the minimization of plant footprint, the performance level is specified as a net plant efficiency greater than 52.5 [\%], and the DOS is discretized using 25 points. Positivity constraints are imposed on the natural gas and steam flows and the initial guess input point is chosen as $(0.1,2.0)$. The obtained outcome from the optimizer as input and output points for this case is presented in Figure 6.5 (see solid red triangles). Specifically, for a natural gas flow of 0.06 [ton/h] and a HRSG steam flow of 0.5 [ton/h], the calculated NGCC modular plant has a net plant power generation of 0.5 
[MW] and a net plant efficiency of 55 [\%]. For this outcome, the operating conditions calculated in Aspen Plus are shown in Table 6.1.

Table 6.1. Operating conditions for intensified modular NGCC plant

\begin{tabular}{|l|c|c|c|c|}
\hline \multirow{2}{*}{ Variable/Stream } & \multicolumn{2}{|c|}{ Gas Turbine } & \multicolumn{2}{c|}{ Steam Turbine } \\
\cline { 2 - 5 } & Inlet & Outlet & Inlet & Outlet \\
\hline Temperature [K] & 1632 & 887 & 839 & 319 \\
\hline Pressure [atm] & 27.4 & 1.0 & 165.4 & 0.1 \\
\hline
\end{tabular}

This result shows that P3 can identify in one step the desired set of conditions for enabling process design and intensification of modular systems. For this NGCC case, the given solution shows that a modular power plant of about 3 orders of magnitude smaller than typical processes in terms of power generation could be enabled if the identified conditions for the combined gas and steam turbine cycles provided by the optimizer were to be employed in the plant design. In particular, for the specified simulation conditions, this result shows the potential for an extreme reduction in size of the NGCC power plant (now $0.5[\mathrm{MW}]$ ) without compromising its high net plant efficiency.

Considering that this P3 framework is a candidate for solving high-dimensional case studies, the next section addresses a DMA-MR $4 \times 4$ system, including the computational time requirements. 
6.3 Exploration of High-Dimensional Application: DMA-MR Case Study (4x4)

The MR operability analysis will be carried out by mapping the feasible regions between the process input and output variables using similar simulation conditions presented in subsection 3.2.1 (DMA-MR Model). For this case study, a $4 \times 4$ system is analyzed. Table 6.2 shows the four selected input/design variables and their available ranges in the AIS. This table also shows the four output variables with their achievable ranges in the AOS, obtained by the operability input-output mapping.

Table 6.2. 4-D DMA-MR system: input-output ranges that define the AIS and AOS

\begin{tabular}{|c|c|}
\hline Input variables & Available ranges \\
\hline Reactor length [cm] & $10-100$ \\
\hline Tube diameter [cm] & $0.5-2.0$ \\
\hline Permeance $\left[\mathrm{mol} /\left(\mathrm{s} . \mathrm{m}^{2} . \mathrm{atm}^{1 / 4}\right)\right]$ & $1 \times 10^{-4}-0.01$ \\
\hline Selectivity [-] & $100-1 \times 10^{5}$ \\
\hline Output variables & Achievable ranges \\
\hline Benzene production [mg/h] & $0-26$ \\
\hline Hydrogen production [mg/h] & $0-7$ \\
\hline Methane conversion [\%] & $10-46$ \\
\hline Cost factor [-] & $100-1,000$ \\
\hline
\end{tabular}

For the performed operability analysis, a grid is built for the input variables by assuming that their ranges are equally divided by 5 , thus 625 points are evaluated. The 
hydrogen production rates are calculated from the permeate side, and a cost factor for comparing different designs is considered, assuming estimated cost parameters associated with the membrane area, the catalyst mass, and the reactor volume. Table 6.3 presents the details for the calculated output ranges including some suggested zones for further analysis.

Table 6.3. 4-D DMA-MR system: achievable ranges for output variables

\begin{tabular}{|c|c|c|c|}
\hline \multirow{2}{*}{$\begin{array}{c}\text { Output } \\
\text { variables }\end{array}$} & \multicolumn{3}{|c|}{ Achievable ranges and zones } \\
\cline { 2 - 4 } & a & b & c \\
\hline Benzene production $[\mathrm{mg} / \mathrm{h}]$ & $10-15$ & $20-26$ & $0-10$ \\
\hline Hydrogen production [mg/h] & $0-4$ & $2-4$ & $4-7$ \\
\hline Methane conversion [\%] & $10-30$ & $40-45$ & $40-46$ \\
\hline Cost factor [-] & $100-300$ & $100-1000$ & $100-1000$ \\
\hline
\end{tabular}

The achievable output ranges are also shown in Figure 6.6 as a 4-D plot, in which the plotted points are color coded with respect to their cost values and some of the $5^{4}$ points considered are superposed. Regarding the specific zones in Figure 6.6, zone "b" represents the best balance between benzene and hydrogen production rates with a high methane conversion, in a wide range of costs. According to the operability analysis, this zone is obtained when the permeance and selectivity are at their highest values, 0.01 [mol/(s.m $\left.\left.\mathrm{m}^{2} \cdot \mathrm{atm}^{1 / 4}\right)\right]$ and 100,000, respectively. This performance level can be explained by the smaller permeation of benzene through the membrane at these conditions, for also small membrane surface areas. For instance, for the mentioned membrane specifications and a tube length and diameter of $10[\mathrm{~cm}]$ and $2[\mathrm{~cm}]$ (reactor volume of $31.4\left[\mathrm{~cm}^{3}\right]$ ), 
respectively, it is possible to obtain production rates of benzene and hydrogen, methane conversion, and cost factor of approx. $25.4[\mathrm{mg} / \mathrm{h}], 3.9[\mathrm{mg} / \mathrm{h}], 40.3[\%]$, and 110, respectively. If the tube length is changed to $100[\mathrm{~cm}]$ (reactor volume of $314.0\left[\mathrm{~cm}^{3}\right]$ ), the calculated production rates of benzene and hydrogen, methane conversion and cost factor reach values of about 24.8 [mg/h], 3.9 [mg/h], 40.4 [\%], and 1,000, respectively.

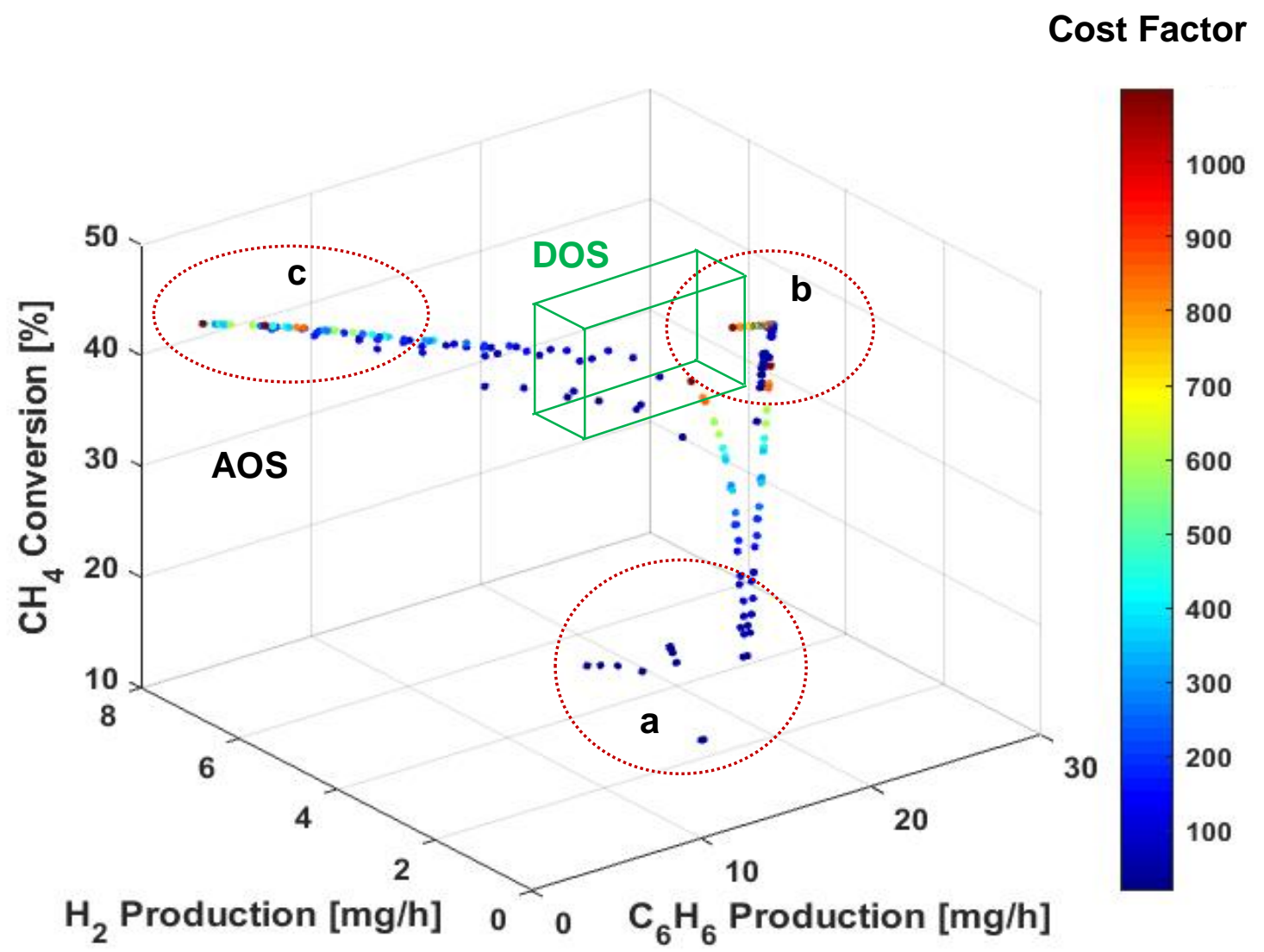

Figure 6.6. 4-D DMA-MR system. AOS and DOS

These results indicate that similar membrane reactor performance levels can be obtained for different footprints (approx. 90\% difference in catalyst volume and membrane surface area between the two cases), and thus cost values. Also, with the operability analysis performed, it is possible to identify that the maximum hydrogen production rate 
in zone "b" is $\approx 4[\mathrm{mg} / \mathrm{h}]$, which could be increased up to $7[\mathrm{mg} / \mathrm{h}]$ in other zones. In contrast, zone "c" shows the lowest benzene production rate caused mainly by low membrane selectivity and high permeance, for reactor length and diameter tending to their high limits. For example, when the membrane permeance, selectivity, tube length and diameter are $0.01\left[\mathrm{~mol} /\left(\mathrm{s} \cdot \mathrm{m}^{2} \cdot \mathrm{atm}^{1 / 4}\right)\right], 100[-], 100[\mathrm{~cm}]$ and $2[\mathrm{~cm}]$, respectively, the results show undesired performance in terms of production rate of benzene (almost zero), for a high methane conversion value of 45.7 [\%]. This outcome suggests as mentioned above that high methane conversions do not necessarily translate to good levels of benzene production rates. Finally, the low methane conversion values for zone "a" occur due to the low membrane permeance considered of $1 \times 10^{-4}\left[\mathrm{~mol} /\left(\mathrm{s} \cdot \mathrm{m}^{2} \cdot \mathrm{atm}^{1 / 4}\right)\right]$. In this case, the reaction equilibrium shift towards products is limited due to the low $\mathrm{H}_{2}$ removal through the $\mathrm{H}_{2}$-selective membrane.

Table 6.4. Computational time for AOS calculations for different DMA-MR subsystems

\begin{tabular}{|c|c|c|}
\hline Subsystems & Points & Time [min:sec] \\
\hline $2 \times 2$ & 25 & $00: 03$ \\
\hline $3 \times 3$ & 125 & $00: 18$ \\
\hline $4 \times 4$ & 625 & $01: 42$ \\
\hline
\end{tabular}

The computational time required to calculate the AOS from the AIS, as a first step in the operability analysis, for the $4 \times 4$ system was 102 seconds as presented in Table 6.4. This table also shows additional information regarding the computational times for different subsystems, 2x2 (inputs: reactor length and diameter; outputs: methane conversion and benzene production) and 3×3 (inputs: reactor length and diameter, and 
membrane selectivity; outputs: methane conversion, benzene and hydrogen productions), of the DMA-MR for comparison. These results indicate that the computation of achievable output regions could be completed in order of minutes or even seconds depending on the considered system dimensionality. All the single core operability calculations in this thesis were carried out on an Intel Core i7 (Sandy bridge) $3.40 \mathrm{GHz}$ processor.

Now, for design and intensification purposes, the DOS is selected (depicted in Figure 6.6 as a green parallelepiped). For this example, the DOS is discretized considering $p=625$ points (i.e., the domains for the benzene and hydrogen production rates, methane conversion, and cost factor are divided in a $5 \times 5 \times 5 \times 5$ grid), thus $k=1,2,3 \ldots 625$. For the DIS* calculation, design constraints associated with the plug flow reactor assumption, such as length over diameter $\geq 30$, reactor length for an experimental setup less than $300[\mathrm{~cm}]$, and membrane permeance and selectivity less than 0.01 [mol/(s.m $\left.\left.\mathrm{m}^{2} \cdot \mathrm{atm}^{1 / 4}\right)\right]$ and 10,000, respectively, are also incorporated into the problem as constraints. Also, positivity restrictions for the input variables are imposed. The optimization problem is solved for all of the DOS points in the established grid.

In order to include desired output requirements in the operability analysis, in this case benzene and hydrogen production rates, as well as methane conversion and cost, the specified DOS is also shown in Table 6.5. Considering that zone "b" presented a good balance among the output variables as discussed above (see Figure 6.6 and Table 6.3), the DOS is defined around this zone. 
Table 6.5. 4-D DMA-MR system: specified DOS, calculated DIS* and DOS* ranges

\begin{tabular}{|c|c|}
\hline Desired output variables & Desired output ranges (DOS) \\
\hline Benzene production [mg/h] & $15-25$ \\
\hline Hydrogen production [mg/h] & $4-5$ \\
\hline Methane conversion [\%] & $35-45$ \\
\hline Cost factor [-] & $100-400$ \\
\hline Feasible input variables & Feasible input ranges (DIS*) \\
\hline Reactor length [cm] & $26-300$ \\
\hline Tube diameter $[\mathrm{cm}]$ & $0.2-1.3$ \\
\hline Permeance $\left[\mathrm{mol} /\left(\mathrm{s} \cdot \mathrm{m}^{2} \cdot \mathrm{atm}^{1 / 4}\right)\right]$ & $4 \times 10^{-4}-0.01$ \\
\hline Selectivity [-] & $72-1 \times 10^{4}$ \\
\hline Feasible output variables & Feasible output ranges (DOS*) \\
\hline Benzene production [mg/h] & $14.0-24.0$ \\
\hline Hydrogen production [mg/h] & $3.9-4.5$ \\
\hline Methane conversion [\%] & $39.9-43.0$ \\
\hline Cost factor [-] & $99.0-400$ \\
\hline
\end{tabular}

In particular, it is desirable to push the production rate of hydrogen as much as possible from the original $2-4[\mathrm{mg} / \mathrm{h}]$ to $4-5[\mathrm{mg} / \mathrm{h}]$ for a reduced cost factor range between 100 - 400. Thus, the desired production levels for hydrogen are outside the original achievable range calculated from the AIS. To provide the feasible operating envelope for the DIS* given the constraints specified by the DOS, the optimization-based operability algorithm defined by problem P3 is employed. Table 6.5 also presents the 
ranges calculated for the DIS* and DOS* as outcomes of P3. The calculated DOS* shows benzene production rate and cost factor ranges that satisfy their respective desired output ranges for the most part. Also, the range of the calculated hydrogen production rate is pushed from $2.0-4.0[\mathrm{mg} / \mathrm{h}]$ to $3.9-4.5[\mathrm{mg} / \mathrm{h}]$ showing an improvement with respect to the original range in zone "b" of the AOS (see Table 6.3). The obtained mapping thus can also provide the input-output points that fall within the desired ranges, for example in zone "b".

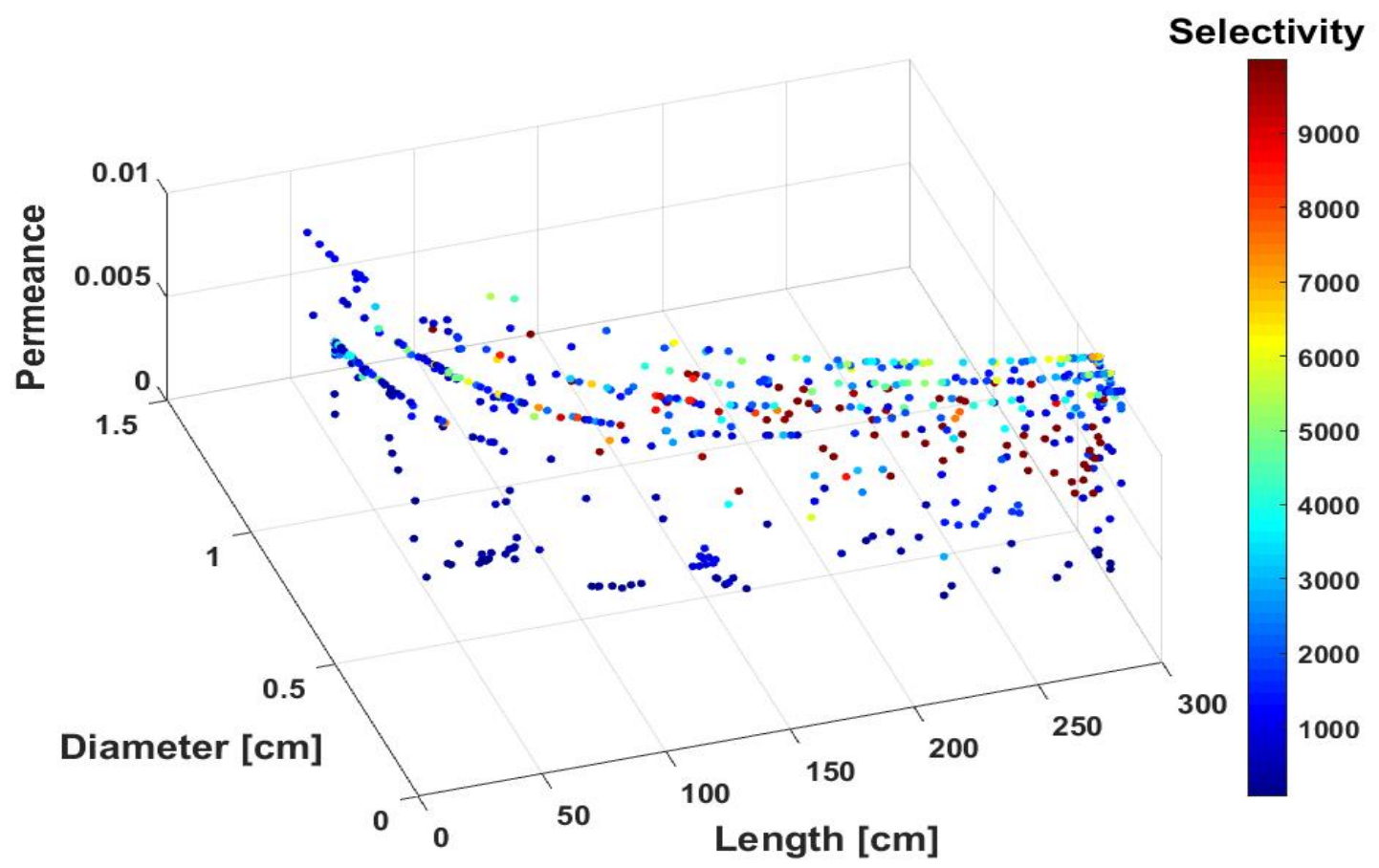

Figure 6.7. 4-D DMA-MR system DIS*

The DIS* outcome from the optimizer for all DOS points is shown in Figure 6.7. Note that the calculated feasible ranges for the input variables are not completely contained in the initial AIS (see Table 6.2 for the AIS ranges). These results thus provide 
the input points that would be necessary to satisfy the DOS, in terms of process design specifications. Therefore, the space DIS* is comprised of the input points that satisfy the specified target specifications and constraints.

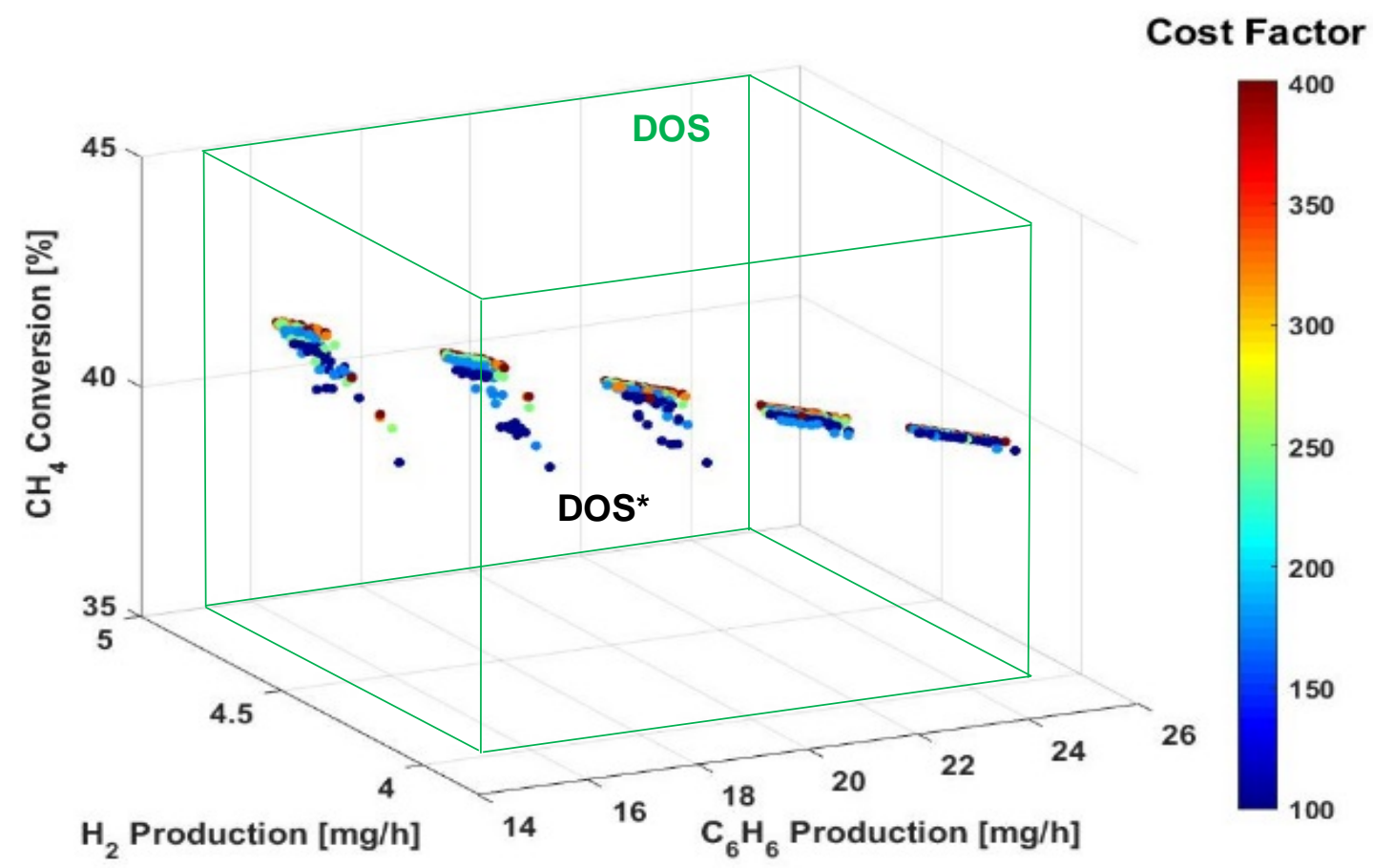

Figure 6.8. 4-D DMA-MR system. DOS and DOS* (zoomed version)

Figure 6.8 depicts the zoomed versions of the DOS (see green parallelepiped) and the calculated DOS* (see solid dots). Table 6.6 shows the intensified input and output variables, considering in P3 a production of benzene greater than $20[\mathrm{mg} / \mathrm{h}]$ and a minimum cost factor, as performance level and process intensification target, respectively. In this case, for a minimum cost factor of approx. 99 [-], the benzene production is around $24[\mathrm{mg} / \mathrm{h}]$. Also, the volume of the reactor is $8.71\left[\mathrm{~cm}^{3}\right]$. When comparing this value to the previously discussed result associated with zone "b", 31.4 
$\left[\mathrm{cm}^{3}\right]$, the current outcome confirms that $\mathrm{P} 3$ is a powerful tool for enabling process intensification.

Table 6.6. 4-D DMA-MR system: intensified input and output points for analyzed variables

\begin{tabular}{|c|c|}
\hline Input variables & Input point \\
\hline Reactor length [cm] & 67.7 \\
\hline Tube diameter [cm] & 0.4 \\
\hline Permeance [mol/(s.m ${ }^{2} \cdot$ atm $\left.\left.^{1 / 4}\right)\right]$ & 0.0073 \\
\hline Selectivity [-] & 9007 \\
\hline Output variables & Output point \\
\hline Benzene production $[\mathrm{mg} / \mathrm{h}]$ & 24.6 \\
\hline Hydrogen production $[\mathrm{mg} / \mathrm{h}]$ & 3.9 \\
\hline Methane conversion [\%] & 39.9 \\
\hline Cost factor [-] & 99.2 \\
\hline
\end{tabular}

Table 6.7 presents the computational time for the DIS* calculations required by P3 to perform the operability computations for the $4 \times 4$ system addressed. This table also shows information for $2 \times 2$ and $3 \times 3$ DMA-MR subsystems. Comparison between the time for DMA-MR $4 \times 4$ calculations, showed in Tables 6.4 and 6.7 , suggests that the computational time for the DIS* calculations is 463 times greater than the time required to calculate the AOS. The high computational time for the 4-D system should not be an issue if offline calculations were to be performed for design and intensification purposes. However, the computational time can significantly increase with system dimensionality, 
resulting in tractability challenges for high-dimensional systems. The reduction of computational time for the calculation of the DIS* from the DOS will be analyzed in the next chapter including mathematical manipulations and parallel computing programming strategies.

Table 6.7. Computational time for DIS* calculations for DMA-MR subsystems of different dimensionalities

\begin{tabular}{|c|c|c|}
\hline Subsystems & Points & Time [hr:min:sec] \\
\hline $2 \times 2$ & 25 & $00: 06: 15$ \\
\hline $3 \times 3$ & 125 & $01: 25: 02$ \\
\hline $4 \times 4$ & 625 & $13: 08: 08$ \\
\hline
\end{tabular}




\section{Chapter 7 Operability Approach for Nonlinear High-Dimensional Systems}

Two strategies towards computational time reduction are introduced in this chapter for the development of an improved operability framework. Using the improved framework, high-dimensional case studies are addressed and computational time analyses are performed.

\subsection{Problem Formulation}

\subsubsection{Strategies towards computational time reduction}

Two approaches are studied for the reduction of the computational time of the bilevel optimization operability framework, problem P3, presented in Chapter 6 . The first considers mathematical manipulations associated with the objective function in the minimization of the relative error (or distance) between the desired output points and the feasible output points. Also, a floating point initial guess for the input to start the optimizer evaluation is analyzed. The use of parallel programming tools in MATLAB is the second strategy employed to reduce the computational time of P3. 


\section{Mathematical manipulations}

The first modification corresponds to employing a new objective function (see Equation 7.1) within argmin of P3. This new function is represented by the absolute value of the relative distance between the desired and feasible output points.

$$
\left\{\sum_{j=1}^{n}\left|\frac{\mathrm{y}_{j, k}-\mathrm{y}_{j, k}^{*}}{\mathrm{y}_{j, k}}\right|\right\}
$$

Note that this objective function is now not continuously differentiable. This modification is not an issue as the bilevel optimization operability approach employs "fminsearch" in MATLAB, which is based on derivative-free optimization tools. After this objective function modification, the computational time for operability calculations of DMAMR subsystems is reduced by around $60 \%$ on average as shown in Table 7.1 , when compared to the original calculated times presented in Table 6.7. This reduction can be explained due to the larger magnitude of the relative error within argmin when the optimizer approaches the minimum. Also, the time reductions in Table 7.1 depend on the search space and the selected DOS.

Table 7.1. Computational time for DIS* calculations for DMA-MR subsystems of different dimensionalities considering modified objective function

\begin{tabular}{|c|c|c|c|}
\hline System & Points & Time [hr:min:sec] & Reduction [\%] \\
\hline $2 \times 2$ & 25 & $00: 02: 55$ & 53 \\
\hline $3 \times 3$ & 125 & $00: 31: 02$ & 63 \\
\hline $4 \times 4$ & 625 & $05: 14: 04$ & 60 \\
\hline
\end{tabular}


The second change for improving P3 corresponds to the use of a smart floating point for the input initial guess to start the optimizer evaluation. In particular, for each new optimization around a specific desired output point, the optimized input point found in the previous iteration is selected as the initial guess for the next iteration. This procedure is repeated until the optimization is completed. This modification further reduces the computational time by around $40 \%$ (see Table 7.2 ), with respect to the improved time showed in Table 7.1.

Table 7.2. Computational time for DIS* calculations for DMA-MR subsystems of different dimensionalities, for improved objective function and floating point for input initial guess

\begin{tabular}{|c|c|c|c|}
\hline System & Points & Time [hr:min:sec] & Reduction [\%] \\
\hline $2 \times 2$ & 25 & $00: 01: 48$ & 38 \\
\hline $3 \times 3$ & 125 & $00: 18: 52$ & 39 \\
\hline $4 \times 4$ & 625 & $03: 05: 45$ & 41 \\
\hline
\end{tabular}

Parallel programming strategy

Parallel programming strategies convert MATLAB applications from serial to parallel by discretization of an algorithm in parts that can be solved concurrently. MATLAB offers two ways for enabling a parallel computing strategy: i) a desktop system with local workers or multicore desktop workers, and ii) a computer cluster with MATLAB Distributed Computing Server, or MATLAB Distributed Computing Server for Amazon EC2. Unfortunately, the floating initial point strategy described above is not compatible with parallel programming, as parallel computing requests that the algorithms are 
independently programmed. Therefore, in this subsection only the objective function modification introduced in Equation 7.1 is considered.

In this thesis, two computer systems are considered for the implementation and analysis of parallel computing: i) desktop system with 4 local cores or workers (Inter Core i7, Sandy bridge, 3.4 GHz processor); and ii) computer cluster with MATLAB Distributed Computing Server, with 63 workers (cores). Figure 7.1 shows the reduction in computational time for DMA-MR subsystems when the desktop system is employed with one (base case), two, three, and four workers.

\begin{tabular}{|c|c|c|c|c|c|}
\hline \multicolumn{3}{|c|}{$\begin{array}{l}\text { Base Case } \\
\text { (1 worker) }\end{array}$} & \multicolumn{3}{|c|}{ (2 workers) } \\
\hline System & Points & $\begin{array}{c}\text { Time } \\
\text { [hr:min:sec] }\end{array}$ & System & Points & $\begin{array}{c}\text { Time } \\
\text { [hr:min:sec] }\end{array}$ \\
\hline $2 \times 2$ & 25 & 00:02:55 & $2 \times 2$ & 25 & 00:01:31 \\
\hline $3 \times 3$ & 125 & 00:31:02 & $3 \times 3$ & 125 & 00:14:59 \\
\hline $4 \times 4$ & 625 & 05:14:04 & $4 \times 4$ & 625 & 02:28:42 \\
\hline \multicolumn{3}{|c|}{ (4 workers) } & \multicolumn{3}{|c|}{ (3 workers) } \\
\hline System & Points & $\begin{array}{c}\text { Time } \\
\text { [hr:min:sec] }\end{array}$ & System & Points & $\begin{array}{c}\text { Time } \\
\text { [hr:min:sec] }\end{array}$ \\
\hline $2 \times 2$ & 25 & 00:00:04 & $2 \times 2$ & 25 & 00:01:02 \\
\hline $3 \times 3$ & 125 & 00:07:51 & $3 \times 3$ & 125 & 00:10:53 \\
\hline $4 \times 4$ & 625 & 01:16:49 & $4 \times 4$ & 625 & 01:44:46 \\
\hline
\end{tabular}

Figure 7.1. Computational time using desktop system with local workers for parallelization

For instance, in the case of DMA-MR $2 \times 2$ system, using the modified objective function in the bilevel optimization operability framework, the computational time has 
decreased from 02:55 [min:sec] to 00:54 [min:sec], when the number of workers increased from 1 to 4 , representing computational time savings of $69 \%$. Similarly, for the DMA-MR $4 \times 4$ system, the computational time is reduced from 05:14:04 [hr:min:sec] to 01:16:49 [hr:min:sec], which shows savings on the order of 4.09 times. These results demonstrate that the proposed parallel programming strategy can successfully reduce the computational time of the bilevel optimization-based operability framework. Taking into consideration parallel computing concepts and tools, a novel bilevel optimizationbased operability framework, problem $\mathrm{P} 4$, is formulated and presented in the next subsection.

\subsubsection{Novel operability framework with parallel computing}

An improved bilevel operability formulation is proposed here based on: i) hierarchical structure for combining P1-P2 in one step (according to subsection 6.1); ii) inner optimization task (argmin) to find the desired input points by minimizing the absolute value of the relative distance between the desired and feasible output points, subject to constraints and target specifications; and iii) parallel computing for converting from serial to parallel algorithms to solve for the total points in the DOS grid. In particular, the inner optimization task is broken down into discrete parts, according to ws MATLAB codes (in

which ws denotes workers or processors) that can be called concurrently. The mathematical representation of the improved bilevel optimization, problem $\mathrm{P} 4$, is as follows: 


$$
\begin{aligned}
& \text { P4: } \quad \Psi=\min _{\mathrm{u} \in \mathbb{U}}\left[\mathrm{PI}_{\text {target }}\right] \\
& \text { subject to: } \mathrm{u}_{k_{w=1}}^{*} \in\left[\underset{\substack{\mathrm{u}_{k_{w}}^{*} \in \mathbb{U}_{k} \\
\mathrm{y}_{k_{w}} \in \text { DOS }}}{\operatorname{argmin}}\left\{\sum_{j=1}^{n}\left|\frac{\mathrm{y}_{j, k_{w}}-\mathrm{y}_{j, k_{w}}^{*}}{\mathrm{y}_{j, k_{w}}}\right|\right\}\right]_{w=1} \\
& \mathrm{u}_{k_{w=2}}^{*} \in\left[\underset{\substack{\mathrm{u}_{k_{w}}^{*} \in \mathbb{U}_{k} \\
\mathrm{y}_{k_{w}} \in \mathrm{DOS}}}{\operatorname{argmin}}\left\{\sum_{j=1}^{n}\left|\frac{\mathrm{y}_{j, k_{w}}-\mathrm{y}_{j, k_{w}}^{*}}{\mathrm{y}_{j, k_{w}}}\right|\right\}\right]_{w=2} \\
& \text {. } \\
& \mathrm{u}_{k_{w=w s}}^{*} \in\left[\underset{\substack{\mathrm{u}_{k_{w}}^{*} \in \mathbb{U}_{k} \\
\mathrm{y}_{k_{w}} \in \mathrm{DOS}}}{\operatorname{argmin}}\left\{\sum_{j=1}^{n}\left|\frac{\mathrm{y}_{j, k_{w}}-\mathrm{y}_{j, k_{w}}^{*}}{\mathrm{y}_{j, k_{w}}}\right|\right\}\right]_{w=w s} \\
& \mathbf{c}_{1}\left(\mathrm{u}_{k}^{*}\right) \leq \mathbf{0}
\end{aligned}
$$

in which $\mathbb{U} \in \mathbb{R}_{\geq 0}^{m}$ and $\mathbb{U}_{k} \subset \mathbb{U}$. Also, the variables are defined as in the description of P3 above. Additionally, $w=1,2,3 \ldots w s$ represents the number of available workers or processors, and $k_{w=1,2,3, \ldots, w s}$ is defined by

$$
k_{w}=\operatorname{round}\left[(w-1) \frac{p}{w s}\right]+1, \ldots, \operatorname{round}\left[w \frac{p}{w s}\right]
$$

For instance, when the number of workers or cores is 4 , and the points in the grid for a 4x4 system $\left(p=5^{4}\right)$ are 625 , each worker can handle on average approx. 156 points:

$$
\begin{gathered}
\text { worker }=1: \quad k_{w=1}=\operatorname{round}\left[(1-1) \frac{625}{4}\right]+1, \ldots, \text { round }\left[1 \frac{625}{4}\right] \\
\qquad k_{w=1}=1, \ldots, 156 \\
\text { worker }=2: k_{w=2}=\operatorname{round}\left[(2-1) \frac{625}{4}\right]+1, \ldots, \text { round }\left[2 \frac{625}{4}\right] \\
k_{w=2}=157, \ldots, 312
\end{gathered}
$$




$$
\begin{gathered}
\text { worker }=3: \quad k_{w=3}=\operatorname{round}\left[(3-1) \frac{625}{4}\right]+1, \ldots, \text { round }\left[3 \frac{625}{4}\right] \\
\qquad k_{w=3}=313, \ldots, 468 \\
\text { worker }=4: k_{w=4}=\operatorname{round}\left[(4-1) \frac{625}{4}\right]+1, \ldots, \text { round }\left[4 \frac{625}{4}\right] \\
k_{w=4}=469, \ldots, 625
\end{gathered}
$$

To solve this bilevel and parallel optimization problem, P4, subroutines available in MATLAB, "fminsearch" and "parpool", are successfully employed for the performed case studies in this chapter. Figure 7.2 depicts the schematic representation of the novel framework for operability to address nonlinear and high-dimensional systems employing bilevel optimization and parallel computing. To assess the impact on computational time of this novel operability framework for process intensification, the next subsection shows new DMA-MR and NGCC case studies of higher dimensionality $5 \times 5$ and $7 \times 3$, and $8 \times 8$ systems, respectively. 


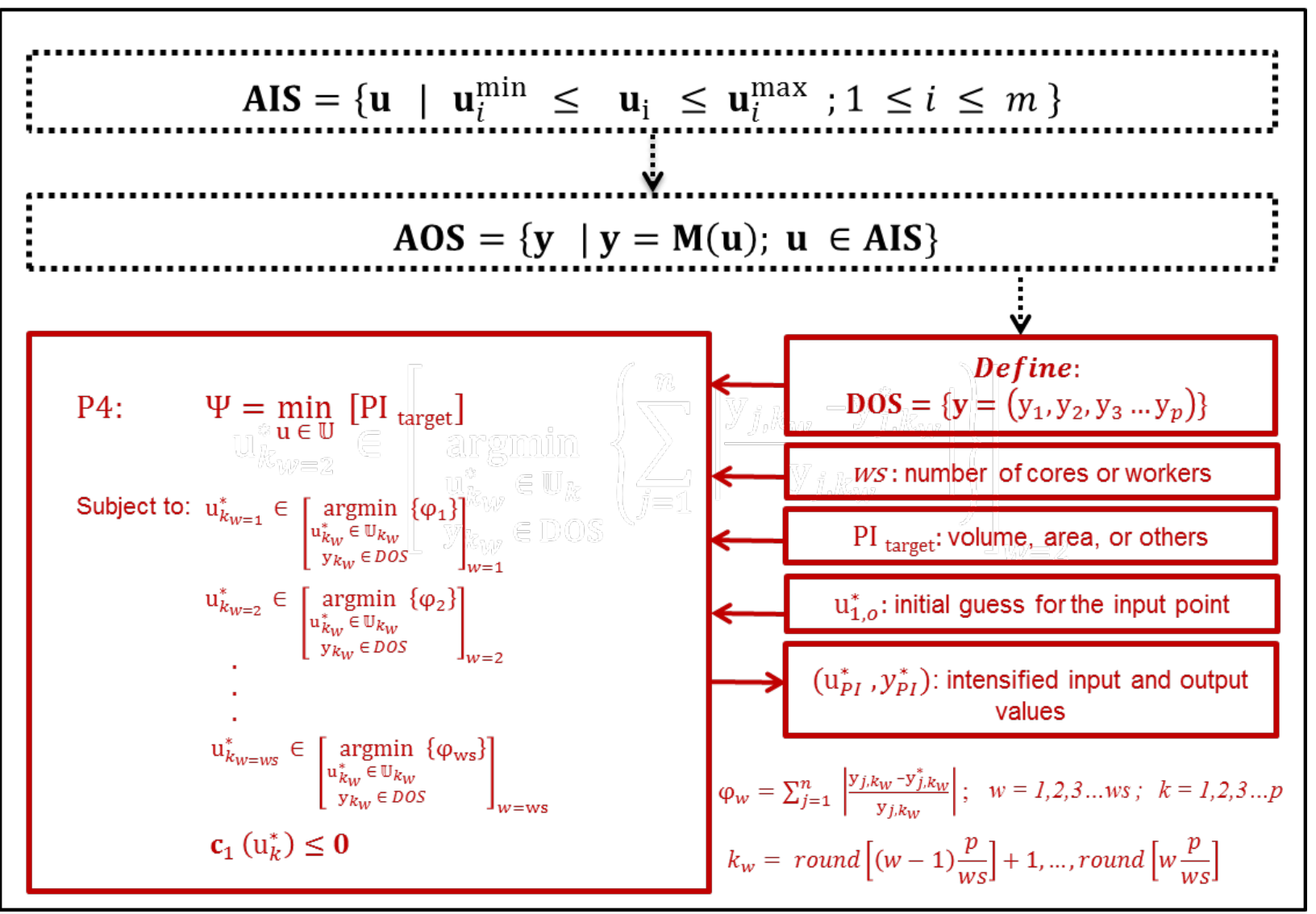

Figure 7.2. Schematic diagram of the bilevel optimization and parallel programming-based operability framework for process intensification

\subsection{Case Studies}

\subsubsection{DMA-MR case study (5x5)}

As the first high-dimensional case study to demonstrate the improved operability framework, a 5x5 DMA-MR system is used for operability calculations and computational time analysis. For this case study, Table 7.3 shows the five selected input/design variables and their available ranges in the AIS. Also, this table depicts the five calculated 
output variables with their achievable ranges in the AOS. Comparing to the $4 \times 4$ case presented above, methane feed is added as a new process input variable to investigate its influence on process intensification targets. Also, methane outlet flow is included as a new output variable. This variable may indicate future modifications to the current DMAMR design towards the implementation of a recycle stream.

Table 7.3. 5-D DMA-MR system: input-output ranges that define the AIS and AOS

\begin{tabular}{|c|c|}
\hline Input variables & Available ranges \\
\hline Reactor length $[\mathrm{cm}]$ & $10-100$ \\
\hline Tube diameter $[\mathrm{cm}]$ & $0.5-2.0$ \\
\hline Permeance $\left[\mathrm{mol} /\left(\mathrm{s} \cdot \mathrm{m}^{2} \cdot \mathrm{atm}^{1 / 4}\right)\right]$ & $1 \times 10^{-4}-0.01$ \\
\hline Selectivity $[-]$ & $100-1 \times 10^{5}$ \\
\hline Methane feed $[\mathrm{mg} / \mathrm{h}]$ & $64-96$ \\
\hline Output variables & Achievable ranges \\
\hline Benzene production $[\mathrm{mg} / \mathrm{h}]$ & $2.2 \times 10^{-5}-28.27$ \\
\hline Hydrogen production $[\mathrm{mg} / \mathrm{h}]$ & $0-7.36$ \\
\hline Methane conversion $[\%]$ & $15.49-51.79$ \\
\hline Cost factor $[-]$ & $18.65-1,100$ \\
\hline Methane outlet flow $[\mathrm{mg} / \mathrm{h}]$ & $1 \times 10^{-4}-81.13$ \\
\hline
\end{tabular}

For the operability analysis, a grid is built for the input variables by assuming that their ranges are equally divided by 5 , thus 3125 points are evaluated. When the achievable output space is of dimension higher than four, a graphical representation for 
analysis of the AOS is not possible. However, the calculated AOS can still provide an insight on how to select the DOS.

Table 7.4 depicts the selected DOS, which presents ranges within the AOS. To provide the feasible operating envelope for the DIS* given the constraints specified by the DOS, the optimization-based operability algorithm defined in P4 is employed. Table 7.4 shows the ranges calculated for the DIS* and DOS* as outcomes of P4. For this example, the DOS is discretized considering $p=3125$ points (i.e., the domains for the benzene and hydrogen production rates, methane conversion, cost factor, and methane outflow are divided in a $5 \times 5 \times 5 \times 5 \times 5$ grid), thus $k=1,2,3 \ldots 3125$. For the DIS* calculation, this example considers similar design constraints as in previous chapters for the DMA-MR associated with the plug flow reactor assumption, such as length over diameter $\geq 30$, reactor length for an experimental setup less than 300 [cm], and membrane permeance and selectivity less than $0.01\left[\mathrm{~mol} /\left(\mathrm{s} \cdot \mathrm{m}^{2} \cdot \mathrm{atm}^{1 / 4}\right)\right]$ and 10,000 , respectively. Positivity restrictions on the input variables are also imposed. 
Table 7.4. 5-D DMA-MR system: specified DOS, calculated DIS* and DOS* ranges

\begin{tabular}{|c|c|}
\hline Desired output variables & Desired output ranges (DOS) \\
\hline Benzene production [mg/h] & $15-25$ \\
\hline Hydrogen production [mg/h] & $5-6$ \\
\hline Methane conversion [\%] & $35-45$ \\
\hline Cost factor [-] & $100-400$ \\
\hline Methane outlet flow [mg/h] & $10-40$ \\
\hline Feasible input variables & Feasible input ranges (DIS*) \\
\hline Reactor length [cm] & $15.50-300$ \\
\hline Tube diameter [cm] & $0.25-1.62$ \\
\hline Permeance $\left[\mathrm{mol} /\left(\mathrm{s} \cdot \mathrm{m}^{2} \cdot \mathrm{atm}^{1 / 4}\right)\right]$ & $7 \times 10^{-5}-0.01$ \\
\hline Selectivity [-] & $17.42-1 \times 10^{4}$ \\
\hline Methane feed $[\mathrm{mg} / \mathrm{h}]$ & $28.8-126.4$ \\
\hline Feasible output variables & Feasible output ranges (DOS*) \\
\hline Benzene production [mg/h] & $2.39-23.63$ \\
\hline Hydrogen production [mg/h] & $2.14-5.99$ \\
\hline Methane conversion [\%] & $34.28-65.14$ \\
\hline Cost factor [-] & $29.97-461.76$ \\
\hline Methane outlet flow [mg/h] & $9.96-63.48$ \\
\hline
\end{tabular}

Note that all the calculated feasible ranges for the input variables are not entirely contained in the initial AIS. For illustration purposes, Table 7.5 presents once again the AIS and the DIS* ranges. In particular, the differences in these ranges can be explained 
by the fact that the DIS* satisfies the specified target and constraints defined for problem P4. For instance, the DIS* calculation includes the target specification associated with the plug flow reactor assumption that is not considered in the original AIS definition.

Table 7.5. 5-D DMA-MR system: summary of input variables- available and feasible ranges

\begin{tabular}{|c|c|c|}
\hline Input variables & $\begin{array}{c}\text { Available } \\
\text { ranges (AIS) }\end{array}$ & $\begin{array}{c}\text { Feasible } \\
\text { ranges (DIS*) }\end{array}$ \\
\hline Reactor length [cm] & $10-100$ & $15.50-300$ \\
\hline Tube diameter [cm] & $0.5-2.0$ & $0.25-1.62$ \\
\hline Permeance [mol/(s.m $\left.\left.{ }^{2} . \mathrm{atm}^{1 / 4}\right)\right]$ & $1 \times 10^{-4}-0.01$ & $7 \times 10^{-5}-0.01$ \\
\hline Selectivity [-] & $100-1 \times 10^{5}$ & $17.42-1 \times 10^{4}$ \\
\hline Methane feed [mg/h] & $64-96$ & $28.8-126.4$ \\
\hline
\end{tabular}

Table 7.6. 5-D DMA-MR system: summary of output variables- available, desired and feasible ranges

\begin{tabular}{|c|c|c|c|}
\hline Output variables & $\begin{array}{c}\text { Achievable } \\
\text { ranges (AOS) }\end{array}$ & $\begin{array}{c}\text { Desired ranges } \\
\text { (DOS) }\end{array}$ & ranges (DOS*) \\
\hline Benzene production [mg/h] & $2 \times 10^{-5}-28.27$ & $15-25$ & $2.39-23.63$ \\
\hline Hydrogen production [mg/h] & $0-7.36$ & $5-6$ & $2.14-5.99$ \\
\hline Methane conversion [\%] & $15.49-51.79$ & $35-45$ & $34.28-65.14$ \\
\hline Cost factor [-] & $18.65-1,100$ & $100-400$ & $29.97-461.76$ \\
\hline Methane outlet flow [mg/h] & $1 \times 10^{-4}-81.13$ & $10-40$ & $9.96-63.48$ \\
\hline
\end{tabular}


A comparison of the output spaces are presented in Table 7.6, where it shows that all the feasible output variables do not satisfy their respective desired output ranges. For instance, the highest feasible methane conversion value of 65.14 [\%] is higher than the original highest achievable value of 51.79 [\%] in the AOS. This conversion value is calculated by problem P4 when the desired output point corresponds to benzene and hydrogen production rates, methane conversion, cost factor, and methane outlet flow of $20[\mathrm{mg} / \mathrm{h}], 5[\mathrm{mg} / \mathrm{h}], 42.5[\%], 400[-]$, and $10[\mathrm{mg} / \mathrm{h}]$, respectively. Note that in this desired output point, the cost factor is the only output at its upper bound due to the large reactor size. Also, the highest methane conversion corresponds to the following feasible input values: reactor length and diameter, membrane permeance and selectivity, and methane feed rate of 212.59 [cm], $0.50[\mathrm{~cm}], 1 \times 10^{-4}\left[\mathrm{~mol} /\left(\mathrm{s} . \mathrm{m}^{2} . \mathrm{atm}^{1 / 4}\right)\right], 9630[-]$, and $40.08[\mathrm{mg} / \mathrm{h}]$, respectively. This result suggests that the length of the reactor, for this point, is the most critical input variable in terms of influencing the cost factor.

Table 7.7 indicates the intensified points for the input and output variables. In this case the calculated reactor volume is of $9.08\left[\mathrm{~cm}^{3}\right]$, which presents a difference of less than $3 \%$ when compared to the 4-D case study (see subsection 6.3.1). 
Table 7.7. 5-D DMA-MR system: intensified input and output variables

\begin{tabular}{|c|c|}
\hline Intensified input variables & Input point \\
\hline Reactor length [cm] & 62.60 \\
\hline Tube diameter [cm] & 0.43 \\
\hline Permeance [mol/(s.m $\left.\left.{ }^{2} . \mathrm{atm}^{1 / 4}\right)\right]$ & $2.9 \times 10^{-3}$ \\
\hline Selectivity [-] & $1.8 \times 10^{3}$ \\
\hline Methane feed [mg/h] & 60.48 \\
\hline Intensified output variables & Output point \\
\hline Benzene production [mg/h] & 20.44 \\
\hline Hydrogen production [mg/h] & 3.71 \\
\hline Methane conversion [\%] & 45.33 \\
\hline Cost factor [-] & 99.68 \\
\hline Methane outlet flow [mg/h] & 32.5 \\
\hline
\end{tabular}

The computational time for the calculation of the DIS* in this 5-D DMA-MR system using the operability framework developed in Chapter 6, problem P3, is approx. 64:54:51 [hr:min:sec]. When the number of workers is 63 , employing P4, this time is reduced to 01:28:57 [hr:min:sec]. Table 7.8 shows the computational times for this 5-D DMA-MR system using the new operability framework, $\mathrm{P} 4$, and the obtained time reduction. 
Table 7.8. Comparison of computational times for P3 and P4 frameworks for the DIS* calculations of the 5x5 DMA-MR system

\begin{tabular}{|c|c|c|c|c|}
\hline System & $\begin{array}{c}\text { Points } \\
\text { DoS }\end{array}$ & $\begin{array}{c}\text { P3 } \\
\text { Time [hr:min:sec] }\end{array}$ & $\begin{array}{c}\text { P4 (63 cores) } \\
\text { Time [hr:min:sec] }\end{array}$ & $\begin{array}{c}\text { Reduction } \\
\text { [times] }\end{array}$ \\
\hline $5 \times 5$ & 3125 & $64: 54: 51$ & $01: 28: 57$ & 43.7 \\
\hline
\end{tabular}

Similar accuracies are obtained when solving P3 or P4 to calculate the DIS* and the DOS* for the same level of performance and intensification target. However, P4 may enable computational time reductions up to 2 orders of magnitude, when compared to the original results using P3.

The next example of a DMA-MR $7 \times 3$ system represents a non-square case that has not been previously explored for process intensification and design purposes using this improved operability framework.

\subsubsection{DMA-MR case study $(7 \times 3)$}

The next case study shows a DMA-MR operability analysis for a system with 7 inputs and 3 outputs. This example is investigated to determine the effect that a nonsquare system has on the operability and computational time outcomes. Specifically, a system with 3 outputs is analyzed to allow its graphical representation for selecting the DOS. The ranges of the 7 selected inputs and the 3 calculated outputs are presented in Table 7.9. 
Table 7.9. $7 \times 3$ DMA-MR system: input-output ranges that define the AIS and AOS

\begin{tabular}{|c|c|}
\hline Input variables & Available ranges \\
\hline Reactor length [cm] & $10-100$ \\
\hline Tube diameter [cm] & $0.5-2.0$ \\
\hline Permeance [mol/(s.m $\left.\left.{ }^{2} . \mathrm{atm}^{1 / 4}\right)\right]$ & $1 \times 10^{-4}-0.01$ \\
\hline Selectivity [-] & $100-1 \times 10^{5}$ \\
\hline Methane feed [mg/h] & $64-96$ \\
\hline Sweep gas feed [mg/h] & $20-40$ \\
\hline Pressure inside tube [atm] & $1-1.2$ \\
\hline Output variables & Achievable ranges \\
\hline Benzene production [mg/h] & $2 \times 10^{-5}-39.35$ \\
\hline Methane conversion $[\%]$ & $15.48-73.89$ \\
\hline Cost factor [-] & $18.65-1,100$ \\
\hline
\end{tabular}

Table 7.10. 7x3 DMA-MR system: achievable ranges for output variables

\begin{tabular}{|c|c|c|c|c|}
\hline \multirow{2}{*}{$\begin{array}{c}\text { Output } \\
\text { variables }\end{array}$} & \multicolumn{4}{|c|}{ Achievable ranges and zones } \\
\cline { 2 - 5 } & a & b & c & d \\
\hline Benzene production [mg/h] & $0-40$ & $0-5$ & $5-15$ & $15-40$ \\
\hline Methane conversion [\%] & $35-74$ & $40-74$ & $15-30$ & $35-65$ \\
\hline Cost factor [-] & $500-1100$ & $50-360$ & $18-200$ & $50-360$ \\
\hline
\end{tabular}

For the performed operability analysis, a grid is built for the input variables by assuming that their ranges are equally divided by 3 , thus 2187 points are evaluated. Table 
7.10 presents the details for the calculated output ranges including suggested four zones for further analysis. The achievable output ranges are also shown in Figure 7.3 as a 3-D plot. In order to include desired output requirements in the operability analysis, in this case benzene production rate, as well as methane conversion and cost, the DOS should now be specified as shown in Table 7.11 and Figure 7.3. Considering that zones "c" and "d" present a good balance among the output variables benzene production and cost, the DOS is defined around these zones. To provide the feasible operating envelope for the DIS* given the constraints specified by the DOS, the optimization-based operability algorithm defined in P4 is employed. For this case study, the DOS is discretized considering $p=125$ points (i.e., the domains for the benzene production rate, methane conversion, and cost factor are divided in a $5 \times 5 \times 5$ grid), thus $k=1,2,3 \ldots 125$.

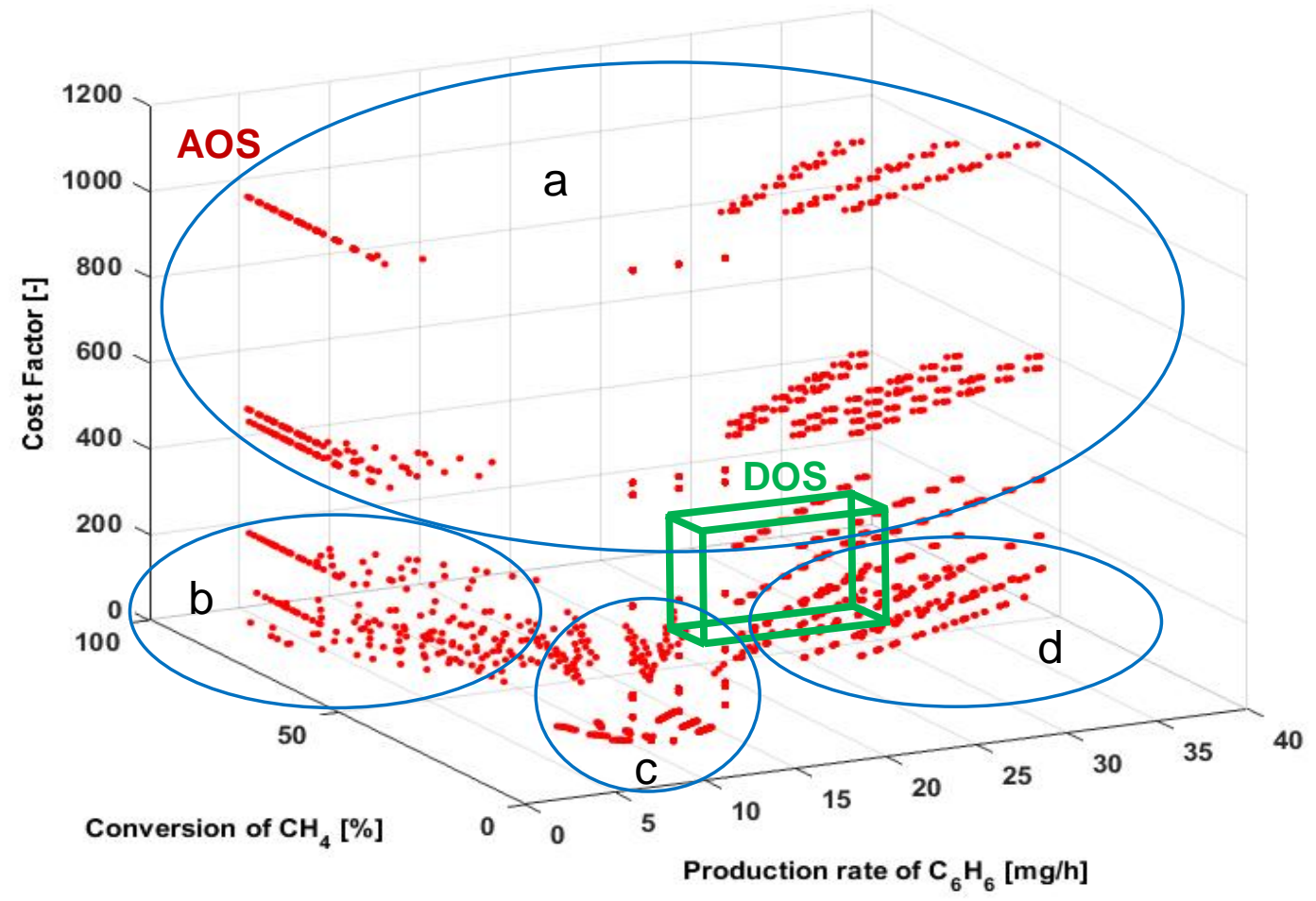

Figure 7.3. $7 \times 3$ DMA-MR system. AOS and DOS 
For the DIS* calculation, similarly to the previous example, design constraints associated with the reactor and membrane are considered. The DOS, along with the ranges calculated for the DIS ${ }^{*}$ and $\mathrm{DOS}^{*}$ as outcomes of $\mathrm{P} 4$ are presented in Table 7.11.

Table 7.11. 7×3 DMA-MR system: specified DOS, calculated DIS* and DOS* ranges

\begin{tabular}{|c|c|}
\hline Desired output variables & Desired output ranges (DOS) \\
\hline Benzene production [mg/h] & $15-25$ \\
\hline Methane conversion [\%] & $35-45$ \\
\hline Cost factor [-] & $100-400$ \\
\hline Feasible input variables & Feasible input ranges (DIS*) \\
\hline Reactor length [cm] & $26-300$ \\
\hline Tube diameter [cm] & $0.26-0.91$ \\
\hline Permeance $\left[\mathrm{mol} /\left(\mathrm{s} \cdot \mathrm{m}^{2} \cdot \mathrm{atm}^{1 / 4}\right)\right]$ & $9 \times 10^{-5}-5.8 \times 10^{-3}$ \\
\hline Selectivity [-] & $59-8,930$ \\
\hline Methane feed [mg/h] & $42.56-103.52$ \\
\hline Sweep gas feed [mg/h] & $12.28-34.64$ \\
\hline Pressure inside tube [atm] & $1.00-1.07$ \\
\hline Feasible output variables & Feasible output ranges (DOS*) \\
\hline Benzene production [mg/h] & $14.99-25.00$ \\
\hline Methane conversion [\%] & $35.00-45.11$ \\
\hline Cost factor [-] & $46.82-400.00$ \\
\hline
\end{tabular}


Regarding the results showed in Table 7.11 , the calculated DOS* satisfy the respective desired output ranges for the most part, with the exception of the lower bound in the cost factor (see Figure 7.4, hollow green circle). In this case, the low cost factor of 46.82 [-] is calculated when the desired output point for benzene production, methane conversion, and cost factor of 15 [mg/h], 35 [\%], and 100 [-], respectively, are used. Also, for this point, the calculated variables in the DIS* correspond to reactor length and diameter, membrane selectivity and permeance, methane and helium inlets, and tube pressure of $25.1[\mathrm{~cm}], 0.5[\mathrm{~cm}], 144.9[-], 1.2 \times 10^{-3}\left[\mathrm{~mol} /\left(\mathrm{s} \cdot \mathrm{m}^{2} . \mathrm{atm}^{1 / 4}\right)\right], 62.24[\mathrm{mg} / \mathrm{h}], 19.72$ [mg/h], and 1 [atm], respectively.

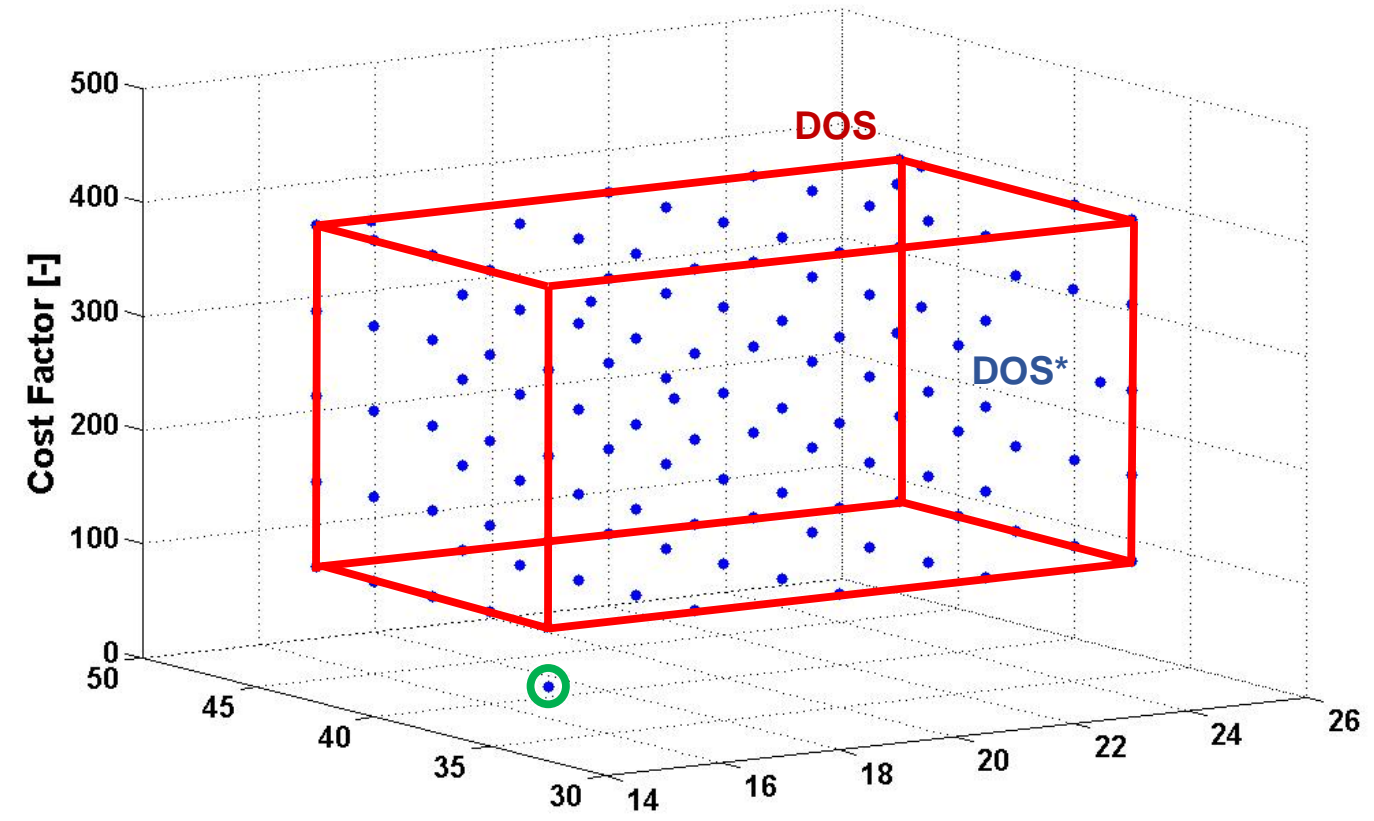

Conversion of $\mathrm{CH}_{4}[\%]$

Production rate of $\mathrm{C}_{6} \mathrm{H}_{6}[\mathrm{mg} / \mathrm{h}]$

Figure 7.4. 7×3 DMA-MR system. DOS and DOS* (zoomed version) 
Figure 7.4 depicts the DOS (see red parallelepiped) and the calculated DOS* (see solid blue dots), which is close to the original grid defined for the DOS. This result shows that for the operability analysis, when the system has more inputs than output variables, the optimization-based operability approach has more independent degrees of freedom to find the points selected from the DOS.

Table 7.12. 7x3 DMA-MR system: intensified input and output variables

\begin{tabular}{|c|c|}
\hline Intensified input variables & Input points \\
\hline Reactor length [cm] & 35.8 \\
\hline Tube diameter [cm] & 0.7 \\
\hline Permeance [mol/(s.m $\left.\left.{ }^{2} \cdot \mathrm{atm}^{1 / 4}\right)\right]$ & $3.2 \times 10^{-3}$ \\
\hline Selectivity [-] & 2093 \\
\hline Methane feed [mg/h] & 4.8 \\
\hline Sweep gas feed [mg/h] & 6.8 \\
\hline Tube pressure [atm] & 1.0 \\
\hline Intensified output variables & Output points \\
\hline Benzene production [mg/h] & 25.0 \\
\hline Methane conversion [\%] & 45.5 \\
\hline Cost factor [-] & 99.9 \\
\hline
\end{tabular}

The intensified input and output variables are shown in Table 7.12. In the previous 5-D DMA-MR example, using P4, the reactor volume was $9.08\left[\mathrm{~cm}^{3}\right]$ and the membrane surface area was $84.52\left[\mathrm{~cm}^{2}\right]$. In this $7 \times 3$ system, the reactor volume and the membrane 
area are $13.79\left[\mathrm{~cm}^{3}\right]$ and $78.80\left[\mathrm{~cm}^{2}\right]$, respectively. Also, for similar values in the methane conversion and cost factor, the intensified benzene production in this $7 \times 3$ system is 25 [mg/h], versus $20.44[\mathrm{mg} / \mathrm{h}]$ calculated for the 5 -D system. This result again suggests that P4 may be able to find points with higher performance when the system has more inputs than output variables, which is expected due to the extra available degrees of freedom.

Table 7.13 depicts a comparison of computational times for the DMA-MR $7 \times 3$ system when calculated using $\mathrm{P} 3$ and $\mathrm{P} 4$ with 63 cores. Note that $\mathrm{P} 4$ presents a reduction of approx. 22 times. Also, this table shows that for the additional DMA-MR $3 \times 3$ and $5 \times 3$ systems, computational time reductions of 36 and 24 times, respectively, are obtained by parallelization. Again, similarly to the $5 \times 5$ example above, Table 7.13 for this case study suggests that the improved operability framework P4 allows reduction of computational times up to 2 orders of magnitude, when compared to the original results with $\mathrm{P} 3$, for similar levels of accuracy in the DIS* and the DOS* computations.

Table 7.13. Comparison of computational times for P3 and P4 frameworks associated with the DIS* calculations for DMA-MR $3 \times 3,5 \times 3$ and $7 \times 3$ subsystems

\begin{tabular}{|c|c|c|c|c|}
\hline Subsystems & $\begin{array}{c}\text { Points } \\
\text { Dos }\end{array}$ & $\begin{array}{c}\text { P3 } \\
\text { Time [hr:min:sec] }\end{array}$ & $\begin{array}{c}\text { P4 (63 cores) } \\
\text { Time [hr:min:sec] }\end{array}$ & $\begin{array}{c}\text { Reduction } \\
\text { [times] }\end{array}$ \\
\hline $3 \times 3$ & 125 & $01: 25: 02$ & $00: 02: 23$ & 36 \\
\hline $5 \times 3$ & 125 & $01: 35: 11$ & $00: 04: 03$ & 24 \\
\hline $7 \times 3$ & 125 & $01: 36: 05$ & $00: 04: 26$ & 22 \\
\hline
\end{tabular}


This non-square case thus indicates that: i) a greater number of inputs than output variables allows the calculation of the DOS* close to the DOS, due to the extra degrees of freedom available to satisfy the points selected within the DOS; and ii) the computational time in P4 is controlled by the number of output variables, and thus additional inputs could be considered for design with reduced increase in computational expense.

In the next case study, the NGCC power plant introduced in Chapters 5 and 6 is revisited considering a high-dimensional system. In this case, operability and computational time analysis are presented for an $8 \times 8$ system, which corresponds to the highest dimensionality supported by the current desktop computer used in this thesis (Inter Core i7, Sandy bridge, 3.4 GHz processor). Due to connection issues between Aspen Plus ${ }^{\circledR}$ and MATLAB $®$ for high-dimensional input ranges, the next NGCC power plant case studies are carried out employing an NGCC first-principles model developed in MATLAB that was validated using Aspen simulations.

\subsubsection{NGCC power plant case study (8x8)}

Starting from the information on the NGCC power plant case study presented in subsection 6.2.2, an $8 \times 8$ system is addressed here. Table 7.14 depicts the selected inputs and the calculated output variables including their ranges. 
Table 7.14. NGCC power plant: input-output ranges that define the AIS and AOS

\begin{tabular}{|c|c|}
\hline Input variables & Available ranges \\
\hline Natural gas feed [ton/h] & $0.08-0.40$ \\
\hline HRSG steam feed [ton/h] & $0.40-4.00$ \\
\hline Compressor outlet pressure [atm] & $10-30$ \\
\hline Air feed temperature [K] & $273-303$ \\
\hline Steam cycle pressure [atm] & $100-200$ \\
\hline Gas turbine efficiency [\%] & $65-85$ \\
\hline Air compressor efficiency [\%] & $65-85$ \\
\hline Steam turbine efficiency [\%] & $65-85$ \\
\hline Output variables & Achievable ranges \\
\hline Net plant power [MW] & $0.09-4.06$ \\
\hline Net plant efficiency [\%] & $7.6-56.5$ \\
\hline Capital cost [\$ millions] & $0.41-8.65$ \\
\hline Gas turbine power [MW] & $0.09-2.23$ \\
\hline Air compressor power [MW] & $0-2.38$ \\
\hline Steam turbine power [MW] & $0.34-4.78$ \\
\hline Flue gas stack temperature [K] & $328-1063$ \\
\hline HRSG net heat [MW] & $0.34-4.78$ \\
\hline
\end{tabular}

A comparison between the $2 \times 2$ and $8 \times 8$ systems presented in Table 7.15 shows that the achievable output ranges for some outputs in the $8 \times 8$ system are wider than the $2 \times 2$ case, due to the influence that the greater number of input variables has in the 
calculations of these output ranges. To obtain a smaller net power plant within the DOS, between $0.1-1.0[\mathrm{MW}]$, the other output ranges are shown in Table 7.16. For such calculations, a grid is built for the selected output variables by assuming that their ranges are equally divided by 5 , thus 390,625 points are evaluated.

Table 7.15. NGCC power plant: comparison between achievable ranges for $2 \times 2$ and $8 \times 8$ systems

\begin{tabular}{|c|c|c|}
\hline $\begin{array}{c}\text { Output } \\
\text { variables }\end{array}$ & $\begin{array}{c}\mathbf{2 \times 2} \text { system } \\
\text { Achievable ranges }\end{array}$ & $\begin{array}{c}\mathbf{8 x 8} \text { system } \\
\text { Achievable ranges }\end{array}$ \\
\hline Net plant power [MW] & $0.50-3.20$ & $0.09-4.06$ \\
\hline Net plant efficiency [\%] & $37.0-55.0$ & $7.6-56.5$ \\
\hline
\end{tabular}

Table 7.16. 8x8 NGCC power plant: achievable and desired output variables

\begin{tabular}{|c|c|c|}
\hline Output variables & Achievable ranges & Desired ranges \\
\hline Net plant power [MW] & $0.09-4.06$ & $0.1-1.0$ \\
\hline Net plant efficiency [\%] & $7.6-56.5$ & $45-60$ \\
\hline Capital cost [\$ millions] & $0.41-8.65$ & $0.5-1.5$ \\
\hline Gas turbine power [MW] & $0.09-2.23$ & $0.1-1.0$ \\
\hline Air compressor power [MW] & $0-2.38$ & $0.1-1.0$ \\
\hline Steam turbine power [MW] & $0.34-4.78$ & $0.1-1.0$ \\
\hline Flue gas stack temperature [K] & $329-1063$ & $329-500$ \\
\hline HRSG net heat [MW] & $0.34-4.78$ & $0.5-4.0$ \\
\hline
\end{tabular}

The improved optimization-based operability algorithm defined in P4 is employed to provide the DIS* given the DOS. Positivity restrictions on the input variables are also 
imposed. Table 7.17 shows the available and calculated desired ranges for the input variables. Once again, the calculated desired ranges for the input variables are not entirely contained in the original AIS.

Table 7.17. 8x8 NGCC power plant: available and desired input variables

\begin{tabular}{|c|c|c|}
\hline Input variables & Available ranges & Desired ranges \\
\hline Natural gas feed [ton/h] & $0.08-0.40$ & $0.01-0.67$ \\
\hline HRSG steam feed [ton/h] & $0.40-4.00$ & $0.09-8.01$ \\
\hline Compressor outlet pressure [atm] & $10-30$ & $1-116$ \\
\hline Air feed temperature [K] & $273-303$ & $328-499$ \\
\hline Steam cycle pressure [atm] & $100-200$ & $0.1-143$ \\
\hline Gas turbine efficiency [\%] & $65-85$ & $70-85$ \\
\hline Air compressor efficiency [\%] & $65-85$ & $70-85$ \\
\hline Steam turbine efficiency [\%] & $65-85$ & $70-85$ \\
\hline
\end{tabular}

Table 7.18 presents the intensified input and output variables. In this case, problem P4 employs as the outer objective function the minimization of the ratio between net plant power over net plant efficiency. The level of performance is specified as a net plant efficiency greater than 45 [\%]. These intensified results suggest that it is possible to calculate a net plant power and efficiency of $0.11[\mathrm{MW}]$ and 56.5 [\%], respectively, with a capital cost of approx. $\$ 0.5$ millions. The capital cost equation for the estimation in this thesis was adapted from DOE and ESPAM (DOE/NETL, 2015; ESMAP, 2009) and can be represented by: Capital Cost $[\$$ millions $]=2.821(N P P)^{0.7991}$, in which NPP is the net plant power [MW]. 
Table 7.18. 8x8 NGCC power plant: intensified input-output variables

\begin{tabular}{|c|c|}
\hline Intensified input variables & Inputs points \\
\hline Natural gas feed [ton/h] & 0.013 \\
\hline HRSG steam feed [ton/h] & 0.157 \\
\hline Compressor outlet pressure [atm] & 5.8 \\
\hline Air feed temperature [K] & 329 \\
\hline Steam cycle pressure [atm] & 140 \\
\hline Gas turbine efficiency [\%] & 70 \\
\hline Air compressor efficiency [\%] & 71 \\
\hline Steam turbine efficiency [\%] & 71 \\
\hline Intensified output variables & Output points \\
\hline Net plant power [MW] & 0.11 \\
\hline Net plant efficiency [\%] & 56.5 \\
\hline Capital cost [\$ millions] & 0.5 \\
\hline Gas turbine power [MW] & 0.09 \\
\hline Air compressor power [MW] & 0.06 \\
\hline Steam turbine power [MW] & 0.08 \\
\hline Flue gas stack temperature [K] & 329 \\
\hline HRSG net heat [MW] & 0.09 \\
\hline & \\
\hline & \\
\hline & \\
\hline & \\
\hline & \\
\hline & \\
\hline & \\
\hline
\end{tabular}

The computational time comparison between P3 and P4 to calculate the DIS* from the DOS for the different NGCC power plant subsystems are showed in Table 7.19. In particular, the estimated time reduction for the $8 \times 8$ system due to parallelization is of 
approx. 70 times. Table 7.19 results also lead to the following conclusions: i) for $2 \times 2$ and $3 \times 3$ systems, the computational time required for the cluster connection is of around 6 seconds; ii) the highest time reduction using P4 is for the $8 \times 8$ system and is of approx. 70 times; and iii) again, for the non-square $7 \times 3$ and $8 \times 3$ systems with reduced number of output variables in the DOS, it is possible to calculate the DIS* with shorter computational times due to the reduced number of outputs.

Table 7.19. NGCC power plant: comparison of computational times for $\mathrm{P} 3$ and $\mathrm{P} 4$ frameworks associated with the DIS* calculations for several subsystems

\begin{tabular}{|c|c|c|c|c|}
\hline Subsystems & $\begin{array}{c}\text { Points } \\
\text { Dos }\end{array}$ & $\begin{array}{c}\text { P3 } \\
\text { Time [hr:min:sec] }\end{array}$ & $\begin{array}{c}\text { P4 (63 cores) } \\
\text { Time [hr:min:sec] }\end{array}$ & $\begin{array}{c}\text { Reduction } \\
\text { [times] }\end{array}$ \\
\hline $2 \times 2$ & 25 & $00: 00: 02$ & $00: 00: 06$ & 0.3 \\
\hline $3 \times 3$ & 125 & $00: 00: 03$ & $00: 00: 06$ & 0.5 \\
\hline $4 \times 4$ & 625 & $00: 00: 38$ & $00: 00: 07$ & 5.4 \\
\hline $5 \times 5$ & 3125 & $00: 05: 06$ & $00: 00: 19$ & 16.1 \\
\hline $7 \times 7$ & 78125 & $02: 58: 42$ & $00: 03: 01$ & 59.2 \\
\hline $7 \times 3$ & 125 & $00: 00: 04$ & $00: 00: 06$ & 0.7 \\
\hline $8 \times 8$ & 390625 & $16: 56: 26$ & $00: 14: 24$ & 70.6 \\
\hline $8 \times 3$ & 125 & $00: 00: 05$ & $00: 00: 06$ & 0.8 \\
\hline
\end{tabular}

The comparison between the improved operability framework, $\mathrm{P} 4$, to $\mathrm{P} 3$, also suggests that: i) for the operability analysis, similar accuracy to calculate the DIS* and the DOS* $^{*}$ is obtained for both problems; and ii) a dramatic reduction in computational time 
can be obtained here, up to 2 orders of magnitude. In the next section, an analysis is introduced towards the exploration of the relationship between computational time and operability calculations for higher dimensions by employing a larger number of cores/workers that may represent a supercomputer.

\subsection{Computational Time Analysis}

\subsubsection{DMA-MR case study}

A summary of the computational times required to perform the operability calculations associated with the DIS* of the DMA-MR subsystems addressed is presented in Table 7.20, considering the MATLAB Distributed Computing Server with 63 workers (cores). A preliminary exploration towards obtaining a relationship between computational time and dimension of the DMA-MR subsystems for operability analysis is performed. In particular, the data shown in Table 7.20 for the $2 \times 2,3 \times 3,4 \times 4$, and $5 \times 5$ square subsystems suggest that it is possible to fit a trendline to extrapolate this relationship. This trendline can be selected between a polynomial of order 2, a power law, or an exponential (see Figure 7.5). For this case, the exponential trendline presents the best coefficient of determination ( $R^{2}$ close to 1 ), which indicates that the relationship between the computational time $(\mathrm{T})$ and dimension of the DMA-MR subsystems (S), for systems with 63 cores, can be represented by

$$
\mathrm{T}_{63}=0.6116 \mathrm{e}^{1.8174 \mathrm{~S}}
$$


Table 7.20. Summary of computational times for DIS* calculations using P4 framework for different DMA-MR subsystems

\begin{tabular}{|c|c|c|}
\hline Subsystems & $\begin{array}{c}\text { Points } \\
\text { Dos }\end{array}$ & $\begin{array}{c}\text { P4 (63 cores) } \\
\text { Time [hr:min:sec] }\end{array}$ \\
\hline $2 \times 2$ & 25 & $00: 00: 23$ \\
\hline $3 \times 3$ & 125 & $00: 02: 23$ \\
\hline $5 \times 3$ & 125 & $00: 04: 03$ \\
\hline $7 \times 3$ & 125 & $00: 04: 26$ \\
\hline $4 \times 4$ & 625 & $00: 14: 54$ \\
\hline $5 \times 5$ & 3125 & $01: 28: 57$ \\
\hline
\end{tabular}

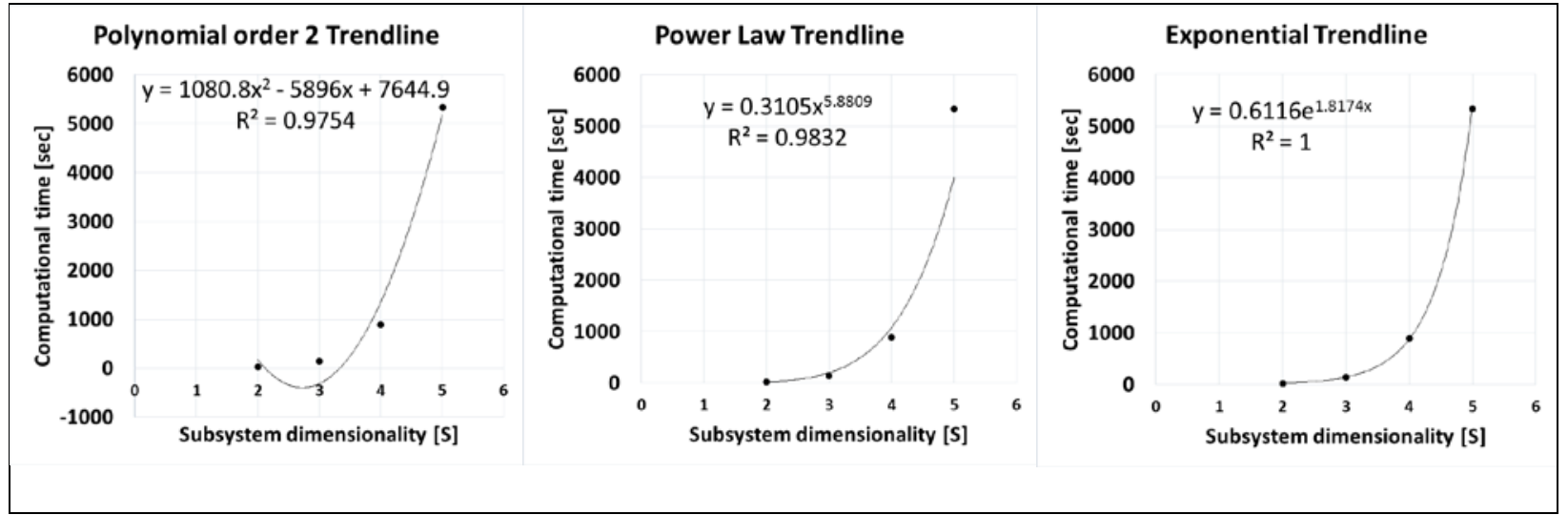

Figure 7.5. Trendlines for the relationship between DMA-MR subsystem dimensionality and computational time for a cluster with 63 cores

Also, a relationship between the number of cores and computational time is presented in Table 7.21 using the available information for the DMA-MR 2x2, 3×3, and 4x4 subsystems. 
Table 7.21. Summary of computational times for the DMA-MR $2 \times 2,3 \times 3$ and $4 \times 4$ subsystems using P4 framework for different number of cores

\begin{tabular}{|c|c|c|c|}
\hline \multirow{2}{*}{$\begin{array}{c}\text { Number of } \\
\text { cores }\end{array}$} & $\mathbf{3}$ Time [hr:min:sec] \\
\cline { 2 - 4 } & $\mathbf{2 \times 2}$ & $\mathbf{3 \times 3}$ & $\mathbf{4 \times 4}$ \\
\hline 1 & $00: 02: 55$ & $00: 31: 02$ & $05: 14: 04$ \\
\hline 2 & $00: 01: 31$ & $00: 14: 59$ & $02: 28: 42$ \\
\hline 3 & $00: 01: 02$ & $00: 10: 53$ & $01: 44: 46$ \\
\hline 4 & $00: 00: 54$ & $00: 07: 51$ & $01: 16: 49$ \\
\hline 63 & $00: 00: 23$ & $00: 02: 23$ & $00: 14: 54$ \\
\hline
\end{tabular}

Considering Table 7.21, the best equation that fits the relationship between the number of cores $(\mathrm{N})$ and computational time for different square subsystems (Ts) is a power law trendline. Figure 7.6 shows the trendlines regarding the DMA-MR $2 \times 2,3 \times 3$, and $4 \times 4$ subsystems, which are represented by $T_{2 \times 2}=126(1 / N)^{0.444}, T_{3 \times 3}=1386(1 / N)^{0.579}$, and $T_{4 \times 4}=14978(1 / N)^{0.704}$, respectively. Note that the power of $T_{s}$ estimation is a function of the DMA-MR dimensionality, for instance, $T_{2 \times 2}$ and $T_{4 \times 4}$ are to the power 0.444 and 0.704 , respectively.

The best trendline that fits this general behavior is represented by

$$
\mathrm{T}_{\mathrm{S}}=\mathrm{BS}_{\mathrm{S}}(1 / \mathrm{N})^{(0.1857+0.13 \mathrm{~S})}
$$

where, Bs is a coefficient that depends on the dimensionality of the DMA-MR system. 


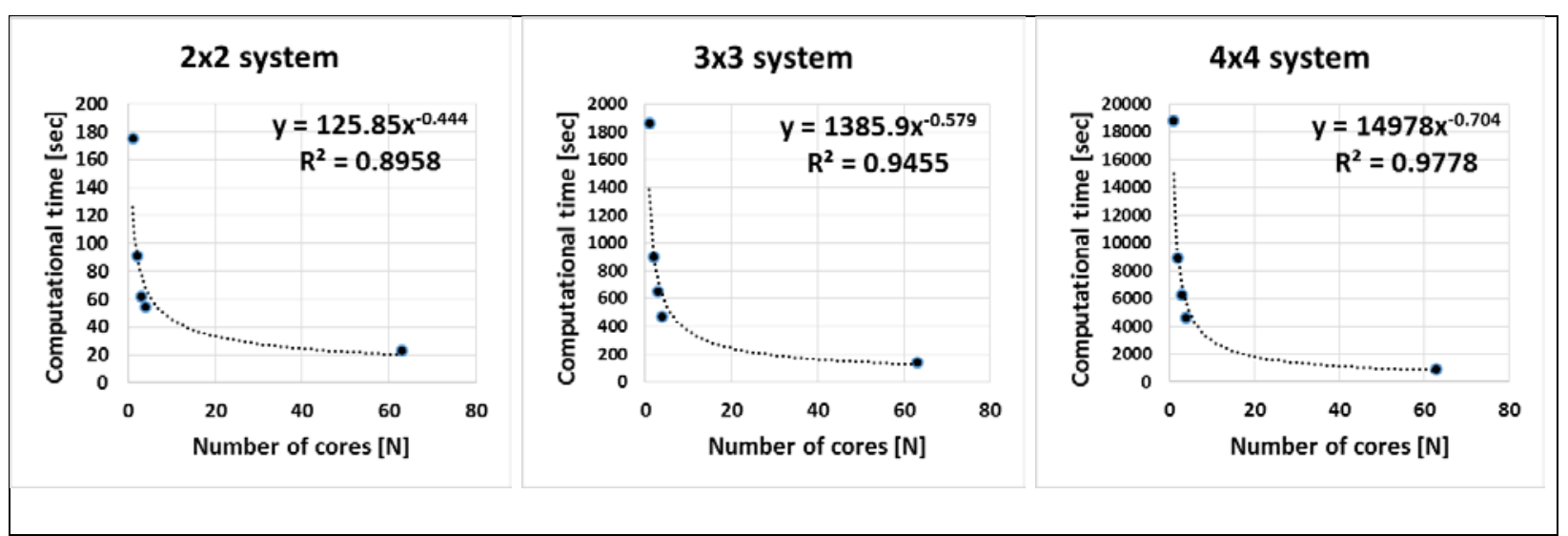

Figure 7.6. Power law trendlines for the relationship between number of cores and computational time for the DMA-MR $2 \times 2,3 \times 3$ and $4 \times 4$ subsystems

Combining both Equations 7.3 and 7.4, and using the computational time for 63 cores as the known boundary values, then $B s=0.6116 \mathrm{e}^{1.8174 \mathrm{~S}}$. Using this result, the following relationship is proposed between the computational time ( $\left.T_{M R}\right)$, dimension of a DMA-MR square subsystem (S), and number of cores in a cluster $(\mathrm{N})$ :

$$
\mathrm{T}_{\mathrm{MR}}(\mathrm{S}, \mathrm{N})=0.6116 \mathrm{e}^{1.8174 \mathrm{~S}}(63 / \mathrm{N})^{(0.1857+0.13 \mathrm{~S})} \quad[\mathrm{sec}]
$$

For instance, a 10x10 DMA-MR system employing 63 cores would need approx. 47 millions of seconds. In contrast, employing for example 16384 cores of the NETL supercomputer "Joule" (Joule is one of the top 100 supercomputers in the world, has 1512 nodes, each node has two 8-core 2.6 GHz Intel Sandy Bridge CPUs, with approx. 17,000 available cores), a computational time of approx. 12,300 seconds would be obtained. Also, in approx. 3,600 seconds, a 9x9 DMA-MR system could be solved in a such a supercomputer. These computational time estimations assume the same computational efficiencies for each node in the supercomputer. 
Another calculation can be carried out when a system with 3 output variables is used for graphical representation, with 3 or more input/design variables. In this case, for 63 workers and using the information showed in Table 7.20 for the DMA-MR $3 \times 3,5 \times 3$, and $7 \times 3$ systems, it is possible to fit a trendline that represents the relationship between the computational time considering 3 outputs in the DOS ( $\left.T_{3 \mathrm{DOS}}\right)$ and different number of input variables (I), following Equation 7.6.

$$
\mathrm{T}_{3 \mathrm{DOS}}(\mathrm{I})=181.42 \ln (\mathrm{I})-54.78 \quad[\mathrm{sec}]
$$

Using this equation for extrapolation purposes, the computational time required for DMA-MR 10x3 and 100x3 subsystems would be of 363 and 781 seconds, respectively. This non-square system analysis suggests once again shorter computational times when the number of desired output variables is reduced as expected due to the presence of the DOS in the objective function. The next subsection presents the computational time analysis for the NGCC power plant when the system increases its dimensionality.

\subsubsection{NGCC power plant case study}

Table 7.22 shows a summary of the computational times required for the operability approach associated with the DIS* calculations of NGCC subsystems using P4 with 63 cores. Note that the computational time for the NGCC $5 \times 5$ system is 380 times faster than the DMA-MR $5 \times 5$ system presented in the previous subsection. This result

suggests that the computational time associated with the operability approach heavily depends on the process model nonlinearities related to each natural gas utilization 
process in focus. The exponential trendline for 63 cores is again obtained to describe the functionality between the computational time and dimension of the square NGCC power plant subsystems.

Table 7.22. NGCC power plant: comparison of computational times using P4 framework for different subsystems

\begin{tabular}{|c|c|c|}
\hline Subsystems & $\begin{array}{c}\text { Points } \\
\text { Dos }\end{array}$ & $\begin{array}{c}\text { P4 (63 cores) } \\
\text { Time [hr:min:sec] }\end{array}$ \\
\hline $2 \times 2$ & 25 & $00: 00: 06$ \\
\hline $3 \times 3$ & 125 & $00: 00: 06$ \\
\hline $4 \times 4$ & 625 & $00: 00: 07$ \\
\hline $5 \times 5$ & 3125 & $00: 00: 19$ \\
\hline $7 \times 7$ & 78125 & $00: 03: 01$ \\
\hline $7 \times 3$ & 125 & $00: 00: 06$ \\
\hline $8 \times 8$ & 390625 & $00: 14: 24$ \\
\hline $8 \times 3$ & 125 & $00: 00: 06$ \\
\hline
\end{tabular}

Figure 7.7 depicts the exponential trendline that represents the relationship between different square NGCC power plant subsystems and computational time. Following a power trendline for the relationship between number of cores and computational time similar to Equation 7.5, Equation 7.7 presents a preliminary estimation of the computational time ( $\mathrm{T}_{\mathrm{NGCC}}$ ) as a function of the NGCC square subsystem dimensionality (S) and number of cores $(\mathrm{N})$ :

$$
\mathrm{T}_{\mathrm{NGCC}}(\mathrm{S}, \mathrm{N})=0.025 \mathrm{e}^{1.3027 \mathrm{~S}}(63 / \mathrm{N})^{(0.1857+0.13 \mathrm{~S})} \quad[\mathrm{sec}]
$$


As an example, an NGCC 10x10 system employing 63 cores would require approx. 11400 seconds. If the NETL supercomputer with 16384 cores was used considering the same assumptions as above, then the computational time needed would be approx. 3 seconds. Also, for the operability calculations of an NGCC $22 \times 22$ system, the computational time required could be of approx. 3,600 seconds. This result as well as the already short computational time of the non-square NGCC systems of around 6 seconds (using 63 cores) indicates that this operability analysis with parallel computing is a solution for high-dimensional nonlinear systems.

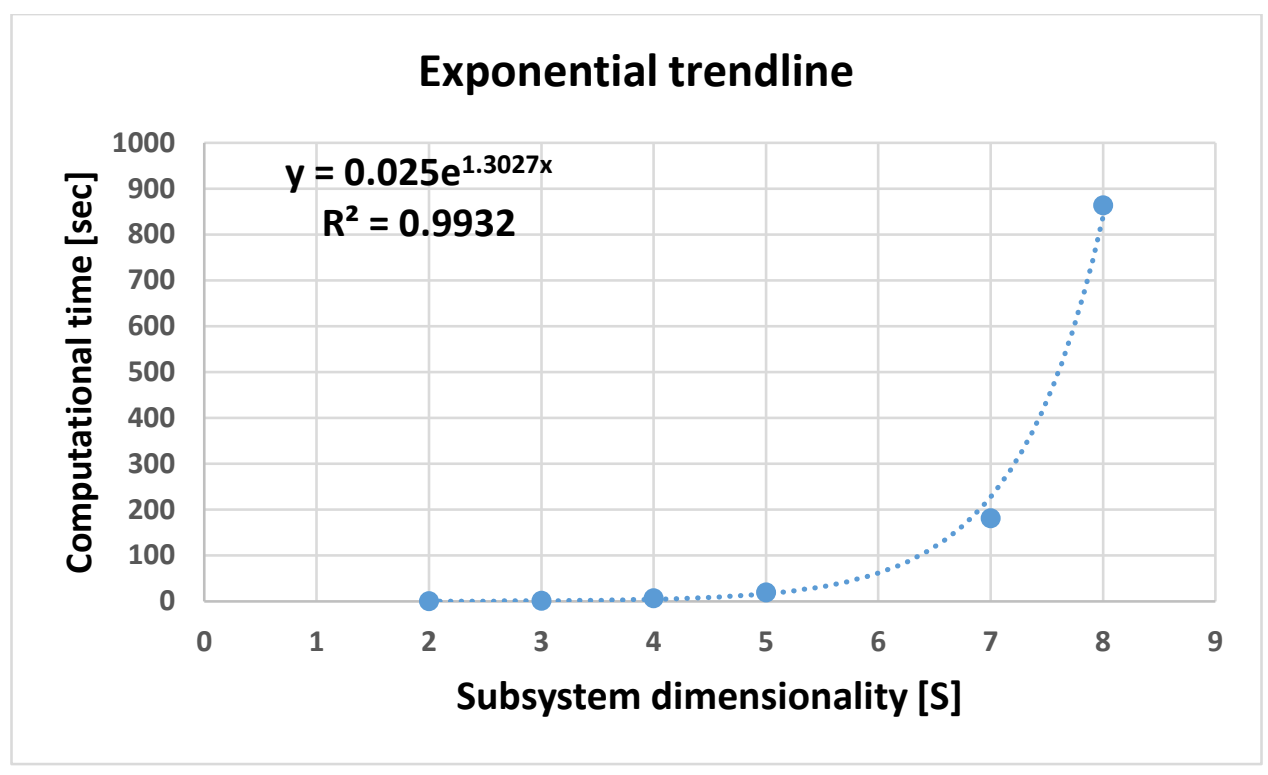

Figure 7.7. Exponential trendline for the relationship between NGCC subsystem dimensionality and computational time for a cluster with 63 cores 


\section{Chapter 8 Conclusions}

In this thesis, the classical operability concepts proposed to verify the ability of a process design, defined by the AIS, to reach an AOS were extended to address design and intensification of chemical and energy systems. As a first step in this extension, a multi-model approach was introduced to calculate the DIS from the DOS within the AOS. In particular, an algorithm was developed using matrix (eigenvalue) properties of each linearized individual system obtained by sensitivity calculations. This algorithm provided a $50 \%$ reduction in the amount of models used to represent the original nonlinear system. The calculation of the DIS for general cases when the AOS does not contain the entire DOS was formulated as an optimization-based operability algorithm, problem $\mathrm{P} 1$. This problem was extended to incorporate process intensification into operability calculations by defining problem P2. P2 allowed the minimization of the selected process intensification target (e.g., volume, area, cost), subject to a desired level of performance.

An integrated optimization-based operability framework P3 was then developed using bilevel tools to enable P1 and P2 calculations in one step, considering defined process design constraints, target specifications, performance levels and intensification targets. The developed framework is flexible, as it allows the calculation of the feasible input points in the DIS* from any DOS employing first-principles models or process simulator runs to obtain the relationship between input and output spaces. In particular, the use of such simulator runs provides a step forward towards the implementation of this

framework for industrial systems that are described by chemical process simulators in general. A catalytic MR for the DMA conversion to benzene and hydrogen, and an NGCC 
system for power generation were analyzed as case studies. These natural gas utilization processes provided individual and integrated nonlinear models for the implementation of the novel framework. The presented results indicated that the developed operability approach can be considered as a powerful tool for the design and intensification of complex energy systems, potentially accelerating the realization of the concept of modular manufacturing.

High computational times required in the DIS* calculations, from the selected DOS, should not be an issue if offline calculations were to be performed for design and intensification purposes especially for low-D systems. A new approach for nonlinear highD systems was developed as problem P4 for DIS* calculations using parallel programming tools. Results on the implementation of the proposed method showed a reduction in computational time up to 2 orders of magnitude, when compared to the original results without parallelization in P3. For parallel computing purposes, the MATLAB Distributed Computing Server at WVU with 63 workers (cores) was employed. In terms of intensification, P4 and P3 presented similar levels of accuracy for the DIS* and the DOS* calculations when the specified constraints and intensification targets are the same. Also, when P4 was considered for non-square case studies with a larger number of inputs than output variables, an important reduction in computational time was obtained. Mathematical relationships using P4 for the operability calculations were also built to estimate the required computational time for high-dimensional systems. Such relationships included: i) dimensionality of chemical and energy systems; and ii) number of cores employed in parallel computing. 
Finally, the results obtained for process design and intensification calculations using P4 showed that the intensified DMA-MR design presented a reduction of approx. $90[\%]$ in reactor volume when compared to the base case. Also, the obtained modular NGCC power plant showed an extreme reduction in size, from 400 to 0.11 [MW], still keeping the plant high net efficiency between 55.0 and 56.5 [\%]. Using the information provided by the optimizer, the user could identify the necessary modifications of the system design that should be performed in order to achieve the feasible targets. For example, for the NGCC case, the developed tool provided the necessary set of design conditions for the successful integration of the gas and steam turbine cycles in order to enable a modular design of a customized size. In conclusion, P1-P4 approaches provide a systematic framework for process intensification of chemical and energy systems towards modularity. 


\section{Chapter 9 Recommendations}

\subsection{Optimization-Based Operability Toolbox}

The operability framework P1-P4 for high-dimensional and nonlinear systems developed in this thesis could be extended to a software package. The package could be incorporated as a toolbox in MATLAB ${ }^{\circledR}$ (the programming language used in this thesis). This toolbox would help to disseminate the benefits of the developed operability approach for process design and intensification in the academy and industrial communities.

\subsection{Multi-Model Operability Approach}

An extension of the multi-model operability approach described in Chapter 4 for high-dimensional nonlinear chemical systems could be an option to take into consideration. A multi-model operability approach could be developed for intensification and modularity by the full utilization of linear programming tools to handle complex systems with the advantage of computational time reduction.

\subsection{Operability Approach and Supercomputer}

The available 63 cores employed for the optimization-based operability analysis was the first effort in operability calculations for high-dimensional nonlinear systems using parallel programming tools. The potentially short computational time obtained with parallel computing suggests that this tool could be a solution for high-dimensional system calculations. Thus, the employment of an external high performance computing center in 
the future (WVU, NETL) should be an excellent path to extend the offline operability analysis to online calculations.

\subsection{Expected Disturbance Set (EDS)}

In this thesis, the expected disturbance set for the operability calculations was assumed with a zero value. For the developed operability framework, this assumption is satisfactory due to the fact that this framework was oriented to process design and intensification. However, the incorporation of the EDS could be used to reflect uncertainties in model parameters employed in the design. This incorporation could open new avenues for process design and intensification under uncertainties. Such set would also be critical when considering the interface between design and control as discussed in the next subsection.

\subsection{Dynamic Operability for Design and Control Interface}

A steady-state operability approach was developed in this thesis. An extension for interfacing design and control could be explored. The AOS could be computed considering the calculated DIS* for different values of the disturbance in the expected disturbance set. Also, process dynamics could be incorporated into the AOS calculation so that a funnel defining the feasible region of operation for design and control could be characterized. This funnel could systematically define the feasible output regions within which chemical or energy systems should be controlled considering the presence of potential disturbances during transient operation. 


\section{References}

Adams, T.A., Barton, P.I., 2010. High-efficiency power production from natural gas with carbon capture. J. Power Sources. 195(7), 1971-1983.

Baldea, M., 2015. From process integration to process intensification. Comput. Chem. Eng. 81, 104-114.

Baliban, R., Elia, J., Floudas, C., 2010a. Toward novel hybrid biomass, coal, and natural gas processes for satisfying current transportation fuel demands, 1: process alternatives, gasification modeling, process simulation, and economic analysis. Ind. Eng. Chem. Res. 49(16), 7343-7370.

Baliban, R., Elia, J., Floudas, C., 2010b. Toward novel hybrid biomass, coal, and natural gas processes for satisfying current transportation fuel demands, 2: simultaneous heat and power integration. Ind. Eng. Chem. Res. 49(16), 7371-7388.

Baliban, R., Elia, J., Floudas, C., 2013. Novel natural gas to liquids processes: process synthesis and global optimization strategies. AIChE J. 59(2), 505-531.

Baliban, R., Elia, J., Misener, R., Floudas, C., 2012. Global optimization of a MINLP process synthesis model for thermochemical based conversion of hybrid coal, biomass, and natural gas to liquid fuels. Comput. Chem. Eng. 42(7), 64-86.

Bard, J., 1998. Practical bilevel optimization: algorithms and applications. The Netherlands: Kluwer.

Bernier, E., Marechal, F., Samson, R., 2010. Multi-objective design optimization of a natural gas-combined cycle with carbon dioxide capture in a life cycle perspective. Energy. 35(2), 1121-1128. 
Boukouvala, F., Muzzio, F., lerapetritou, M., 2010. Design space of pharmaceutical processes using data-driven-based methods. J. Pharm. Innov. 5(3), 119-137.

Boukouvala, F., lerapetritou, M., 2012. Feasibility analysis of black-box processes using an adaptive sampling Kriging-based method. Comput. Chem. Eng. 36(1), 358-368.

Boukouvala, F., Dubey, A., Vanarase, A., Ramachandran, R., Muzzio, F., lerapetritou, M., 2012. Computational approaches for studying the granular dynamics of continuous blending processes, 2-population balance and data-based methods. Macromol. Mater. Eng. 297(1),9-19.

Brunetti, A., Caravella, A., Drioli, E., Barbieri, G., 2012. Process intensification by membrane reactors: high-temperature water gas shift reaction as single stage for syngas upgrading. Chem. Eng. Technol. 35(7), 1238-1248.

Capstone Turbine Corporation, 2016. Fallowfield gas processing plant. http://content.stockpr.com/capstoneturbine/db/185/904/pdf/CS_CAP411_Fallowfield _lowres.pdf (accessed 09.10.16).

Chen, Z., Chen, X., Shao, Z., Yao, Z., Biegler, L.T., 2013. Parallel calculation methods for molecular weight distribution of batch free radical polymerization. CACE. 48: 175186.

Colson, B., Marcotte, P., Savard, G., 2007. An overview of bilevel optimization. Annals of Operational Research. 153, 235-256.

Conner, J., Manousiouthakis, V., 2013. On the attainable region for process networks. AIChE J. 60(1), 193-212.

DOE, 2015. Process intensification workshop report from Advanced Manufacturing Office September 29-30, Alexandria, VA. 
DOE/NETL, 2013. Current and future technologies for natural gas combined cycle (NGCC) power plants. Technical report, DOE/NETL-341/061013.

DOE/NETL, 2015. Cost and performance baseline for fossil energy plants. Volume 1a: bituminous coal (PC) and natural gas to electricity. Technical report revision 3, DOE/NETL-2015/1723, 2015.

Dominguez L., Pistikopoulos, E., 2013. A quadratic approximation-based algorithm for the solution of multiparametric mixed-integer nonlinear programming problems. AIChE J. 59(2), 483-495.

Drioli, E., Stankiewicz, A., Macedonio, F., 2011. Membrane engineering in process intensification - An overview. J. Membr. Sci. 380(1-2), 1-8.

EIA, 2016a. U.S. Energy Information Administration. Natural gas report. http://www.eia.gov/dnav/ng/ng_sum_Isum_dcu_nus_m.htm (accessed 09.10.16).

EIA, 2016b. U.S. Energy Information Administration. Today in energy. http://www.eia.gov/todayinenergy/detail.cfm?id=25392 (accessed 09.10.16).

Elvidge, C.D., Ziskin, D., Baugh, K.E., Tuttle, B.T., Ghosh, T., Pack, D.W., Erwin, E.H., Zhizhin, M., 2009. A fifteen year record of global natural gas flaring derived from satellite data. Energies. 2, 595-622.

ESMAP, 2009. ESMAP Technical Paper 122/09: study of equipment prices in the power sector. https://openknowledge.worldbank.org/bitstream/handle/10986/17531/81725 OESMOESMAOBox0379844B0OPUBLIC0.pdf?sequence=1 (accessed 01.21.17).

Fesko, O., 2012. A parallel approach to improvement and estimation of the approximate optimal control. Journal of Computer Science. 3:486-491. 
Georgakis, C., Li, L., 2010. On the calculation of operability sets of nonlinear highdimensional processes. Ind. Eng. Chem. Res. 49(17), 8035-8047.

Georgakis, C., Uzturk, D., Subramanian, S., Vinson, D.R., 2003. On the operability of continuous processes. Control Eng. Prac. 11 (8), 859-869.

GPA (2010). Gas Processors Association (GPA), Cooperative Research 2010 advancing the midstream industry. https://gpaglobal.org/assets/gpaglobal/gpa/pdf/research/ CooperativelndustryBrochure_09.pdf. (accessed 09.10.15).

G-Team A.S., 2016. Steam Turbines. http://www.steamturbo.com/steam-turbines.html (accessed 09.10.16).

High, K.A. and LaRoche, R.D., 1995. Parallel nonlinear optimization techniques for chemical process design problems. Computers chem. Engng. (19) 6/7: 807-825.

Joel, A.S., Wang, M., Ramshaw, C., Oko, E., 2014. Process analysis of intensified absorber for post-combustion CO2 capture through modelling and simulation. Int. J. Greenhouse Gas Control. 21, 91-100.

Joel, A.S., Wang, M., Ramshaw, C., 2015. Modelling and simulation of intensified absorber for post-combustion CO2 capture using different mass transfer correlations. Applied Thermal Engineering. 74, 47-53.

Kehlhofer, R., Bert, R., Frank, H., Franz, S., 2009. Combined-cycle gas \& steam turbine power plants.

Khajuria, H., Pistikopoulos, E., 2013. Optimization and control of pressure swing adsorption processes under uncertainty. AIChE J. 59(1), 120-131.

Kreith, F., Goswami, D., 2007. Handbook of energy efficiency and renewable energy. CRC Press. New York, USA. 
Kvamsdal, H.M., Hetland, J., Haugen, G., Svendsen, H.F., Major, F., Karstad, V., Tjellander, G., 2010. Maintaining a neutral water balance in a 450 MW NGCC-CCS power system with post-combustion carbon dioxide capture aimed at offshore operation. Int. J. Greenhouse Gas Control. 4(4), 613-622.

Leblebici, M., Stefanidis, G., Van Gerven, T., 2015. Comparison of photocatalytic spacetime of 12 reactor designs for wastewater treatment. Chem. Eng. Process. Process Intensif. 97, 106-111.

Li, L., Borry, R., Iglesia, E., 2002. Design and optimization of catalysts and membrane reactors for the non-oxidative conversion of methane. Chem. Eng. Sci. 57(21), 45954604.

Li J, Yoon H, Wachsman E. Hydrogen permeation through thin supported

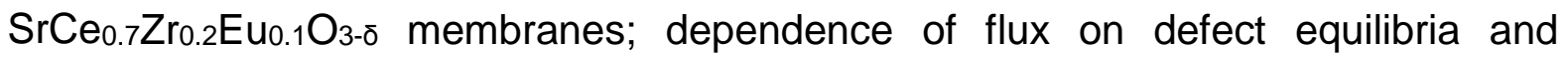
operating conditions. J. Membrane Sci. 2011;381(1-2):126-131.

Lier, S., Wörsdörfer, D., Grünewald, M., 2016. Transformable production concepts: flexible, mobile, decentralized, modular, fast. ChemBioEng Reviews, 3(1), 16-25.

Liese, E.A., Zitney, S.E., 2013. A dynamic process model of a natural gas combined cycle - model development with startup and shutdown simulations. In Proceedings of the ASME 2013 Power Conference, Boston, MA.

Lima, F.V., Georgakis, C., 2008. Design of output constraints for model-based nonsquare controllers using interval operability. J. Proc. Cont. 18(6), 610-620.

Lima, F.V., Georgakis, C., 2010. Input-output operability of control systems: the steadystate case. J. Proc. Cont. 20(6), 769-776. 
Lima, F.V., Georgakis, C., Smith, J.F., Schnelle, P.D., Vinson, D.R., 2010a. Operabilitybased determination of feasible control constraints for several high-dimensional nonsquare industrial processes. AIChE J. 56(5), 1249-1261.

Lima, F.V., Jia, Z., lerapetritou, M., Georgakis, C., 2010b. Similarities and differences between the concepts of operability and flexibility: the steady-state case. AIChE J. 56(3), 702-716.

Liu, P., Pistikopoulos, E., Li, Z., 2009. An energy systems engineering approach to polygeneration and hydrogen infrastructure systems analysis \& design. Chem. Eng. Trans. 18(5), 373-378.

Locatelli, G., Bingham, C., Mancini, M., 2014. Small modular reactors: a comprehensive overview of their economics and strategic aspects. Progress in Nuclear Energy. 73, 75-85.

Ma, Y., Shao, Z., Chen, X., Biegler, L.T., 2016. A parallel function evaluation approach for solution to large-scale equation-oriented modles. CACE. 93: 309 -322.

Malcolm, A., Polan, J., Zhang, L., Ogunnaike, B., Linninger, A., 2007. Integrating systems design and control using dynamic flexibility analysis. AIChE J. 53(8), 2048-2061.

Manassaldi, J.I., Mores, P.L., Scenna, N.J., Mussati, S., 2014. Optimal design and operating conditions of integrated plant of NGCC and postcombustion CO 2 capture. Ind. Eng. Chem. Res. 53(44), 17026-17042.

Mathieu, R., Pittard, L., Anandalingam, G., 1994. Genetic algorithm based approach to bi-level linear programming. Operations Research. 28(1), 1-21. 
MathWorks, 2017. Perform parallel computations on multicore computers, GPUs, and computer clusters. https://www.mathworks.com/products/parallel-computing.html (accessed 04.17.17)

Mokhatab S, Poe W. Handbook of natural gas transmission and processing. Elsevier, Waltham, MA, Second Edition, 2012.

Moon, J., Kim, S., Linninger, A., 2011a. Integrated design and control under uncertainty: embedded control optimization for plantwide processes. Comput. Chem. Eng. 35(9), 1718-1724.

Moon, J., Kim, S., Linninger, A., 2011b. Embedded control for optimizing flexible dynamic process performance. Ind. Eng. Chem. Res. 50(9), 4993-5004.

National Science Foundation (NSF), 2014. Process Intensification Workshop Report September 30-October 1, Washington, DC.

NaturalGas.org, 2016. Microturbines. http://naturalgas.org/overview/uses-electrical/ (accessed 09.10.16).

Nord, L.O., Anantharaman, R., Bolland, O., 2009. Design and off-design analyses of a pre-combustion CO 2 capture process in a natural gas combined cycle power plant. Int. J. Greenh. Gas Cont. 3(4), 385-392.

Ramshaw, C., 1995. The incentive for process intensification. Proceedings, 1st Intl. Conf. Proc. Intensif. for Chem. Ind., 18, BHR Group, London, p. 1.

Rawlings, J., Ekerdt, J., 2002. Chemical reactor analysis and design fundamentals; Nob Hill: Madison, WI, USA.

Roy, S., 2007. Zeton Inc. Process Development: Consider modular plant design. Chemical Engineering Progress. 113(5): 28-31. 
Santoso H, Hioe D, Bao J, Lee P. Operability analysis of nonlinear processes based on incremental dissipativity. J. Proc. Cont. 2012;22(1):156-166.

Setiawan R, Bao J. Plantwide operability assessment for nonlinear processes using a microscopic level network analysis. Chem. Eng. Res. Des. 2012;90(1):119-128.

Sinha, A., Malo, P., Frantsev, A., Deb K., 2013. Finding optimal strategies in multi-period multi-leader-follower stackelberg games using evolutionary framework. Computers and Operations Research. Elsevier.

Sinha, A., Malo, P., Deb K., 2014. Test problem construction for single-objective bilevel optimization. Evolutionary Computational Journal. 22(3), 439-477.

Sirkar, K., Fane, A., Wang, R., Wickramasinghe S., 2015. Process intensification with selected membrane processes. Chem. Eng. Process. Process Intensif. 87, 16-25.

Stankiewicz, A., Moulin, J., 2000. Process intensification: transforming chemical engineering. Chem. Eng. Prog. 96(1), 22-34.

Velez, S., Maravelias, C., 2013. A branch-and-bound algorithm for the solution of chemical production scheduling MIP models using parallel computing. CACE. 55:2539.

Vinson, D., Georgakis C., 2000. A new measure of process output controllability. J. Proc. Cont. 10(2-3), 185-194.

Wang, M., Joel, A.S., Ramshaw, C., Eimer, D., Musa, N.M., 2015. Process intensification for post-combustion $\mathrm{CO}_{2}$ capture with chemical absorption: a critical review. Applied Energy. 158, 275-291. 
Weng, J., Chen, X., Biegler, L.T., 2015. A multi-thread parallel computation method for dynamic simulation of molecular weight distribution of multisite polymerization. CACE. 82: $55-67$.

Wörsdörfer, D., Lier, S., Grünewald, M., 2015. Potential analysis model for case specific quantification of the degree of eligibility of innovative production concepts in the process industry. Chem. Eng. Process. Process Intensif. 98, 123-136.

Yin, Y., 2000. Genetic algorithm based approach for bilevel programming models. Jounal of Transportation Engineering. 126(2), 115-120. 